\title{
Diversity and distribution of Finnish aphyllophoroid and heterobasidioid fungi (Basidiomycota): An update
}

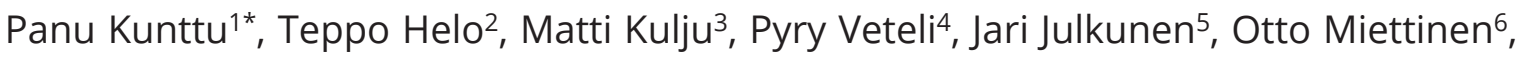 \\ Jorma Pennanen7, Aki Moilanen ${ }^{8}$ \& Heikki Kotiranta9
}

\section{Article info}

Received: 29 Apr. 2021

Revision received: 1 Jul. 2021

Accepted: 14 Jul. 2021

Published: 30 Jul. 2021

\section{Associate Editor}

Bryn T. M. Dentinger

\begin{abstract}
Biogeographical and ecological knowledge of aphyllophoroid fungi has increased substantially after the publication of the Finnish aphyllophoroid checklist. In this paper, we describe the occurrence and distributions of both aphyllophoroid and heterobasidioid fungi in Finland. We introduce 13 species new to Finland: Hyphoderma lapponicum, Mycostilla vermiformis, Proterochaete adusta, Pseudotomentella alobata, Pseudoxenasma verrucisporum, Sistotrema subtrigonospermum, Spiculogloea minuta, Tomentella botryoides, Tomentella neobourdotii, Tomentella subtestacea, Tomentella subpilosa, Tulasnella anguifera, and Tulasnella interrogans. Proterochaete and Pseudoxenasma are new genera to Finland. We also present the record of Caudicicola gracilis for only the second time globally. Furthermore, we present 115 new records (locations) of 53 rare or seldom collected species. In addition, we report 96 species considered new to a specific subzone of the boreal forest vegetation zone in Finland. The records contain notes on the substrata, and the ecology and distribution of nationally new species and are briefly discussed.
\end{abstract}

Key words: Aphyllophorales, biogeography, corticioids, polypores, wood-associated fungi

\section{Introduction}

The checklist of Finnish aphyllophoroid fungi was published in 2009 (Kotiranta et al. 2009). Since that time, substantial new information on both aphyllophoroid and heterobasidioid species has accumulated, which includes a total of 102 species new to Finland (H. Kotiranta, unpublished), 406 records of rare species, and 525 species new to some region (e.g., Kunttu et al. 2016, 2018, 2019). The species that are new to Finland can be either factually new finds or results of better understanding of species identity due to taxonomic revisions. This accumulated knowledge is mainly explained by recent extensive field inventories and studies, while areas (e.g., Kainuu province, Oulu region) and substrata (e.g., fine woody debris) that had

\footnotetext{
${ }^{1}$ Hertsbölentie 8 H, FI-25900 Taalintehdas, Finland

2 Erätie 13 C 19, FI-87200 Kajaani, Finland

${ }^{3}$ Biodiversity Unit, P.O. Box 3000, FI-90014 University of Oulu, Finland

${ }^{4}$ Helsinki, Finland

${ }^{5}$ University of Eastern Finland, Faculty of Health Sciences, School of Medicine, Institute of Dentistry, P.O. Box 1627, FI-70211 Kuopio, Finland

${ }^{6}$ Finnish Museum of Natural History, P.O. Box 7, FI-00014 University of Helsinki, Finland

${ }^{7}$ Keskuskatu 68 A 9, FI-10300 Karjaa, Finland

${ }^{8}$ Sääskensuontie 227 A, FI-90310 Oulu, Finland

${ }^{9}$ Finnish Environment Institute, Latokartanonkaari 11, FI-00790 Helsinki, Finland

*Corresponding author, e-mail: panu.kunttu@iki.fi
}

previously received little attention have also been newly surveyed (e.g., Juutilainen 2016).

In the current paper, we present new records of aphyllophoroid and heterobasidioid fungi in Finland, where we consider three types of records: 1) species new to Finland, 2) rare or seldom collected species with a maximum of 10 previous records in Finland, and 3) species that are new to a specific subzone (section) of the boreal vegetation zone in Finland.

We considered both aphyllophoroid and heterobasidioid fungi in their broader sense, approximately following Hansen and Knudsen (1997), with the exception that the gastromycetoid genera were excluded. Although we concentrated mainly on corticioid fungi, the heterobasidioid genera (such as Helicogloea, Spiculogloea, and Tulasnella) were also included, since they were also included in the Finnish checklist of aphyllophoroid fungi (Kotiranta et al. 2009).

\section{Material and methods}

Most of the records in this paper were derived by mycologists from field trips and species inventories (e.g., Finnish Atlas of Fungi), but some old herbarium specimens were also studied. A common inventory method was an opportunistic search/sampling for species, which entailed a careful walk through a study site to collect visible sporocarps, 
and emphasis was placed on sampling multiple habitats and substrate qualities to collect a large number of species and to obtain a representative picture of the species composition of the study site (Stokland \& Sippola 2004). Field work by the author PV during 2020 in Lapland was conducted in the form of systematic inventory of sample plots. The work was part of a Natural Resources Institute Finland LUKE led project. The majority of the records date from 2019 and 2020, but some older records were also included.

At the beginning of each fungal record, we provide the biogeographical provinces according to Knudsen and Vesterholt (2008), and Latin province names are presented on the website of FinBIF (2021a) alongside this provincial division. For each record, we named the sites at two or three levels: a municipality and a village, or a topographical site or nature conservation area. If the biogeographical province was the same as in the previous record for the same fungal species, it was not repeated. The Finnish National Uniform Coordinate System (UCS, $27^{\circ} \mathrm{E}$; Heikinheimo \& Raatikainen 1981) was used to present the coordinates. The forest vegetation subzones (Fig. 1) follow Ahti et al. (1968), and are also available on the online FinBIF map (FinBIF 2021b). Following the abovementioned sources, the names of the subzones were presented in English (e.g., Ostrobothnia), in contrast to the Latin names that were used for the biogeographical provinces (e.g., Ostrobottnia).

Taxonomy and nomenclature mainly follow Kotiranta et al. (2009) and Hjortstam and Ryvarden (2009), but Bernicchia and Gorjón (2010) was also used for some species, as was Spirin et al. (2019a) for genus Protomerulius, and Svantesson et al. (2019) for the Pseudotomentella tristis group. The Finnish Red List assessment of species according to the IUCN-standard corresponds to Kotiranta et al. (2019). If a species status was determined to be of least concern, it was not mentioned.

The decay stage classification (1-5) of dead wood was carried out according to Renvall (1995), with stage 1 referring to hard dead wood and stage 5 referring to completely decayed wood. This is widely used method for all tree species in the boreal forests (e.g., Korhonen 2009; Siitonen et al. 2009). The diameter of dead wood was measured at chest height if the trunk was complete and from the middle if it was broken. Here, we use the Finnish term 'kelo' to refer to dead and old-growth trunks of Scots pine (Pinus sylvestris) with a silvery-grey, decorticated appearance. Kelo trees are special substrata for fungi due to their extremely slow decay rate (even hundreds of years), long-lasting hard surface and chemical compounds (Leikola 1969; Niemelä et al. 2002; Venugopal et al. 2016). Classification of habitat types follows, to a large extent, the Red List assessment of habitat types in Finland (Kontula \& Raunio 2018).

The material were collected, identified, and confirmed by several mycologists as described in the record details, using the following abbreviations: $\mathrm{TH}=$ Teppo Helo, $\mathrm{JJ}=$ Jari Julkunen, HK = Heikki Kotiranta, MK = Matti Kulju, $\mathrm{OM}=$ Otto Miettinen, $\mathrm{AM}=$ Aki Moilanen, $\mathrm{JP}=$ Jorma Pennanen, and PV $=$ Pyry Veteli. Unless otherwise

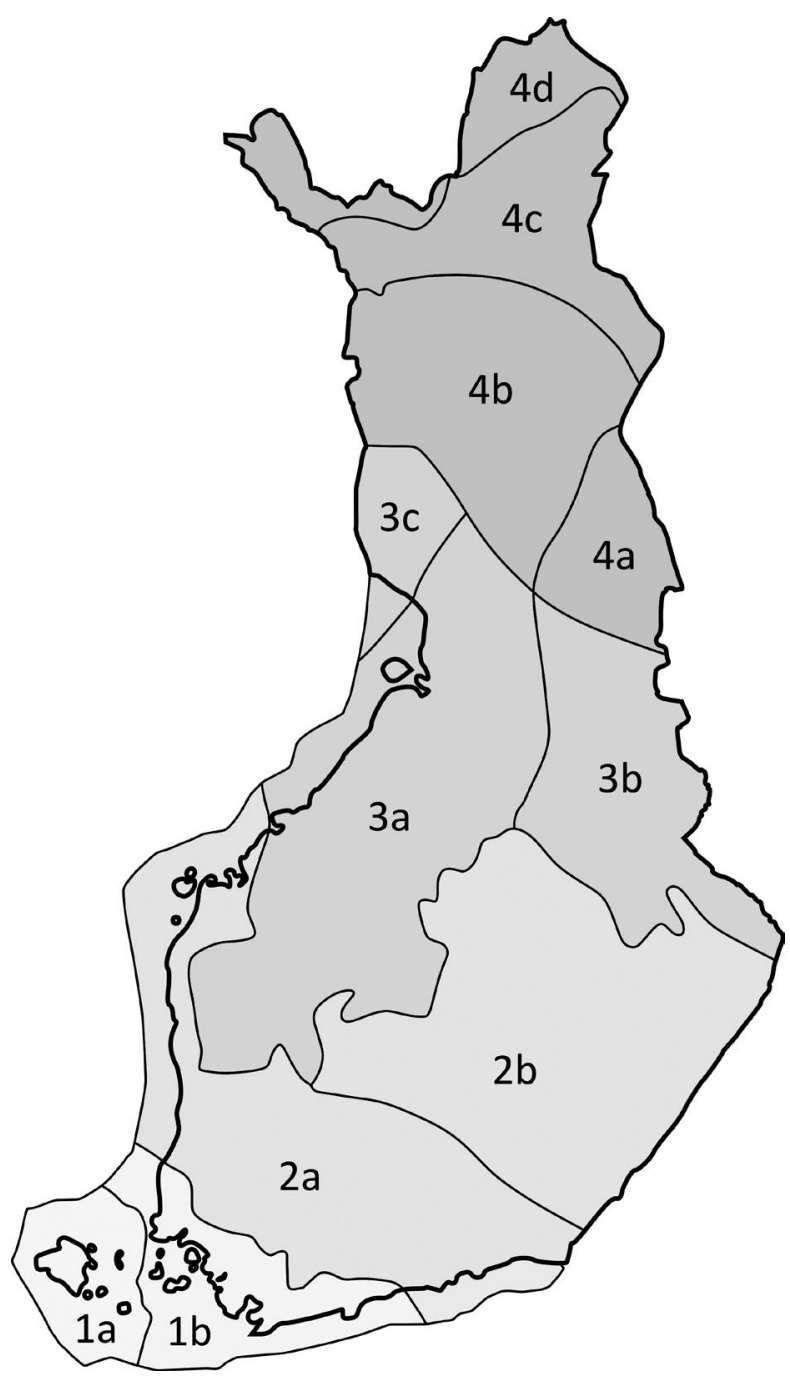

Figure 1. Boreal forest vegetation zones (1-4) and their subzones in Finland. 1a - Hemiboreal, Åland; 1b - Hemiboreal, Oak zone; 2a Southern boreal, Southwestern Finland, and Southern Ostrobothnia; 2b - Southern boreal, Lake district; 3a - Middle boreal, Ostrobothnia; 3b - Middle boreal, Northern Carelia - Kainuu; 3c - Middle boreal, Southwestern Lapland; $4 \mathrm{a}$ - Northern boreal, Kuusamo District; $4 \mathrm{~b}$ Northern boreal, North Ostrobothnia; $4 \mathrm{c}$ - Northern boreal, Forest Lapland; 4d - Northern boreal, Fjeld Lapland.

stated, the collector was also the identifier. The code after the name of the collector represents the personal sampling number of the specimen. Voucher specimens were deposited in the herbaria of the universities of Helsinki (H), Oulu (OULU), Turku (TUR), Jyväskylä (JYV) and Kuopio Natural History Museum (KUO).

\section{Results}

We discovered 13 fungal species new to Finland: Hyphoderma lapponicum, Mycostilla vermiformis, Proterochaete adusta, Pseudotomentella alobata, Pseudoxenasma verrucisporum, Sistotrema subtrigonospermum, Spiculogloea minuta, Tomentella botryoides, Tomentella neobourdotii, Tomentella subtestacea, Tomentella subpilosa, Tulasnella anguifera, and Tulasnella interrogans. We also present the record of Caudicicola gracilis for only the second time globally. Furthermore, 115 new records (locations) of 53 rare or seldom collected species (with a maximum 
Table 1. Number of the new species in subzones of the boreal forest vegetation zone.

\begin{tabular}{l|c}
\hline Subzone of boreal forest vegetation zone & $\begin{array}{c}\text { Number of new } \\
\text { species }\end{array}$ \\
\hline Hemiboreal, Oak zone (1b) & 5 \\
Southern boreal, Southwestern Finland and & 3 \\
$\quad$ Southern Ostrobothnia (2a) & 7 \\
Southern boreal, Lake district (2b) & 19 \\
Middle boreal, Ostrobothnia (3a) & 42 \\
Middle boreal, Northern Carelia - Kainuu (3b) & 11 \\
Middle boreal, Southwestern Lapland (3c) & 2 \\
Northern boreal, Kuusamo District (4a) & 4 \\
Northern boreal, North Ostrobothnia (4b) & 4 \\
Northern boreal, Fjeld Lapland (4d) & \\
\hline
\end{tabular}

of ten previous records in Finland) are presented. Altogether, 96 species are reported as new to a specific subzone (section) of the boreal forest vegetation zone in Finland (Table 1).

The species are listed below in alphabetical order.

Amaurodon cyaneus (Wakef.) Kõljalg \& K.H. Larsson (Fig. 2)

Distribution. New to 3 b (Fig. 1).

Notes. $3^{\text {rd }}$ record in Finland; previous records: Helsinki (1b) (Kotiranta et al 2009). Vulnerable.

Specimen examined. Ostrobottnia kajanensis, Sotkamo, Losonvaara, UCS 7107057:3546404, on a fallen trunk of Populus tremula (diam. $8 \mathrm{~cm}$, decay stage 2) in an old spruce-dominated herb-rich heath forest, 6 Oct. 2018, leg. \& det. TH 20180214 (OULU), conf. MK.

\section{Amylocorticium pedunculatum Hjortstam}

(Fig. 3)

Distribution. New to 3 b (Fig. 1).

Notes. $5^{\text {th }}-7^{\text {th }}$ records; previous records: Helsinki (1b), Lammi (2a), Luhanka (2b), and Äänekoski (2b) (Kotiranta et al. 2009; H. Kotiranta, unpublished).

Specimens examined. Ostrobottnia kajanensis, Kuhmo, Jämäsvaara SE, UCS 7099282:3624258, on a fallen small trunk of Populus tremula (diam. $12 \mathrm{~cm}$, decay stage 4) in a mixed mesic heath forest, 8 Sept. 2016, leg. \& det. JP3182 (H); Sotkamo, Naulavaara, UCS 7087212:3559108, on fallen trunk of Picea abies (diam. $5 \mathrm{~cm}$, decay stage 3), in a middle-aged mixed herbrich heath forests, 11 Sept. 2012, leg. P. Helo 2343 (OULU), det. TH; Sotkamo, Losonvaara, UCS 7106778:3545296, on a fallen trunk of Picea abies (diam. $10 \mathrm{~cm}$, decay stage 3), in an old spruce-dominated mesic heath forest, 30 Sept. 2020, leg. \& det. TH $20200015(\mathrm{H})$.

Amyloxenasma allantosporum (Oberw.) Hjortstam \& Ryvarden

Distribution. New to 3a (Fig. 1).

Specimen examined. Ostrobottnia ouluensis, Oulu, Karjasilta, UCS 7212887:3429705, on a stump of Larix sibirica (decay stage 2), in a small mesic heath forest with larch-trees planted next to the road, 26 Oct. 2019, leg. \& det. AM 244 (OULU), conf. MK.

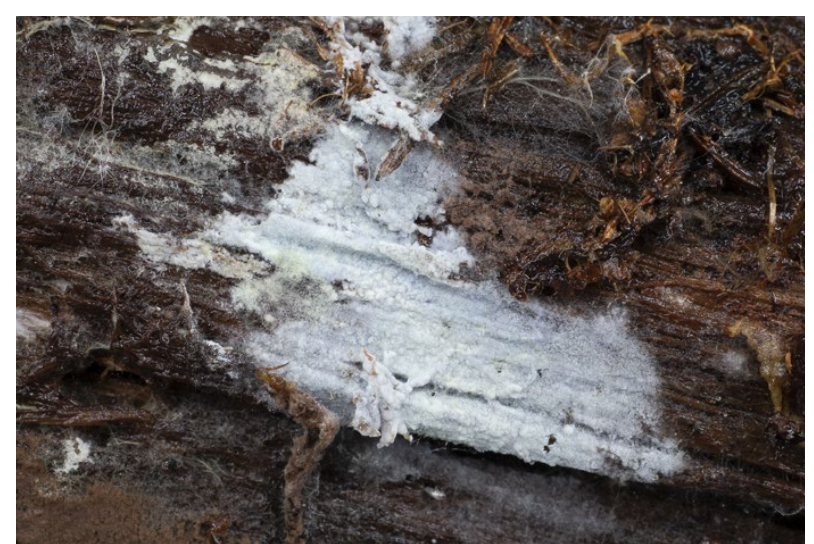

Figure 2. Amaurodon cyaneus in Sotkamo (TH 20180214). Photo: Teppo Helo.

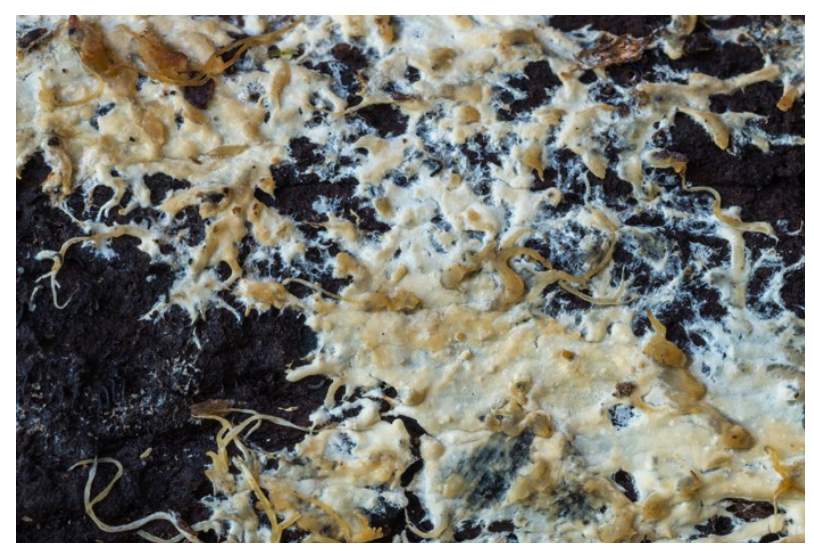

Figure 3. Amylocorticium pedunculatum in Kuhmo, ex situ (JP3182). Photo: Jorma Pennanen.

\section{Antrodia leucaena Y.C. Dai \& Niemelä}

(Fig. 4)

Notes. $10^{\text {th }}$ record in Finland; previous records: Raasepori (1b), Sipoo (2a), Porvoo (2a), Hyvinkää (2a), Padasjoki (2a), Hirvensalmi (2b), Ruovesi (2b), Savonranta (2b), and Hyrynsalmi (3b) (Spirin et al. 2013; Kunttu et al. 2016; FinBIF 2021c). Vulnerable.

Specimen examined. Nylandia, Helsinki, Kustaankartano, UCS 6681:3386, on fallen, decorticated trunk of Populus tremula (diam. $14 \mathrm{~cm}$, decay stage 3 ) in former agricultural land, now herb-rich forest with high amounts of dead wood, dominated by Salix caprea, Alnus incana and Ulmus glabra, 17 Oct. 2020, leg. \& det. PV 2570 (H), conf. OM.

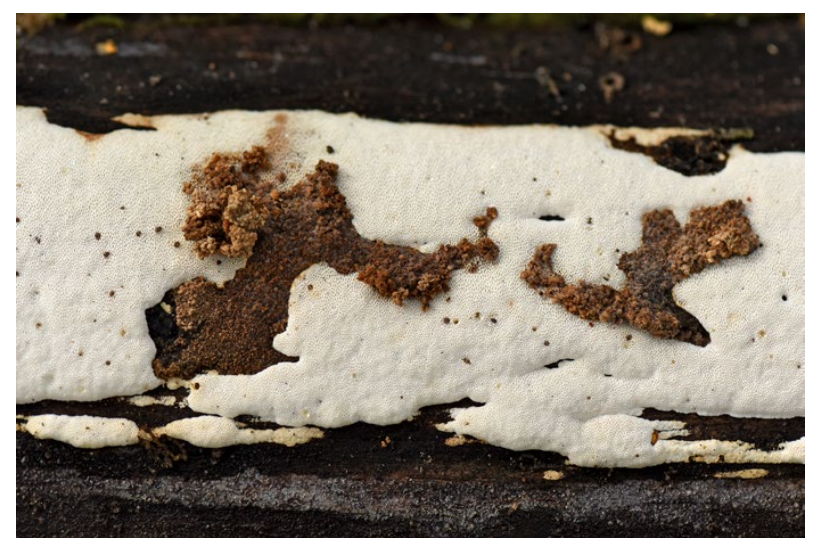

Figure 4. Antrodia leucaena in Helsinki (PV 2570). Photo: Pyry Veteli. 


\section{Antrodiella parasitica Vampola}

(Fig. 5)

Distribution. New to $2 b$ (Fig. 1).

Notes. $8^{\text {th }}$ and $9^{\text {th }}$ records in Finland; previous records: Karjalohja (1b), Lammi (2a), Padasjoki (2a), Sipoo (2a; two sites), Vantaa (2a), and Puolanka (3b) (Kotiranta et al. 2009; Kunttu et al. 2014). Vulnerable.

Specimens examined. Nylandia, Helsinki, Kustaankartano, UCS 6681:3386, on fallen Picea abies (diam. 35 cm, decay stage 2) in association with dead and live Trichaptum abietinum basidiomata, in Picea abies dominated old stand with high amount of coarse dead wood, 11 Oct. 2020, leg. \& det. PV $2557(\mathrm{H})$, conf. OM; Savonia borealis, Iisalmi, Poskimäki, UCS 7053991:3511039, on a fallen trunk of Picea abies (diam. $10 \mathrm{~cm}$, decay stage 2) in a pine-dominated rocky forest, 9 Sept. 2020, leg. O. Ryhänen 1/9920 (OULU), det. MK, conf. HK.

Aphanobasidium subnitens (Bourd. \& Galz.) Jülich

Distribution. New to $3 b$ (Fig. 1).

Notes. $3^{\text {rd }}$ record in Finland; previous records: Luhanka (2a) and Porvoo (2a). (Kotiranta et al. 2009; Kunttu et al. 2018).

Specimen examined. Ostrobottnia kajanensis, Kuhmo, Ulvinsalo, UCS 7102091:3663174, on a fallen trunk of Picea abies (diam. $35 \mathrm{~cm}$, decay stage 4), in a very old dwarf shrub spruce mire, 5 Oct. 2020, leg. \& det. TH 20200029 (OULU, H), conf. MK, HK \& V. Spirin.

\section{Athelia acrospora Jülich}

Distribution. New to 3c (Fig. 1).

Specimen examined. Ostrobottnia ultima, Tervola, Pisavaara Strict Nature Reserve, Vähäloma, UCS 7353720:3412120, on a kelo branch of Pinus sylvestris on the ground (diam. $5 \mathrm{~cm}$, decay stage 1) in an old stony mesic heath forest, 4 Oct. 2020, leg. \& det. MK 67/20 (OULU).

\section{Athelia bombacina (Pers.) Jülich}

Distribution. New to 3c (Fig. 1).

Specimens examined. Ostrobottnia ultima, Tervola, Pisavaara Strict Nature Reserve, Vähäloma, UCS 7352735:3413712, on a decorticated fallen trunk of Pinus sylvestris (diam. $25 \mathrm{~cm}$, decay stage 3 ) in an old pine-dominated mesic heath forest, 4 Oct. 2020, leg. \& det. MK 58/20 (OULU); Ostrobottnia ultima, Tervola, Pisavaara Strict Nature Reserve, UCS 7353868:3411948, on a partly decorticated fallen trunk of Picea abies (diam. $23 \mathrm{~cm}$, decay stage 4) in an old spruce-dominated mesic heath forest, 4 Oct. 2020, leg. \& det. MK 68/20 (OULU).

Athelopsis glaucina (Bourdot \& Galzin) Parmasto

Notes. $9^{\text {th }}$ record in Finland; previous records: Geta (1a), Finström (1a), Helsinki (1b), Tavastia australis (2a/2b), two records in 3a, and records in 3c and 4c (FinBIF 2021c, H. Kotiranta, unpublished).

Specimen examined. Nylandia, Karjaa, Lepinjärvi, UCS 6664384:3313649, on a fallen branch of Corylus avellana (diam.

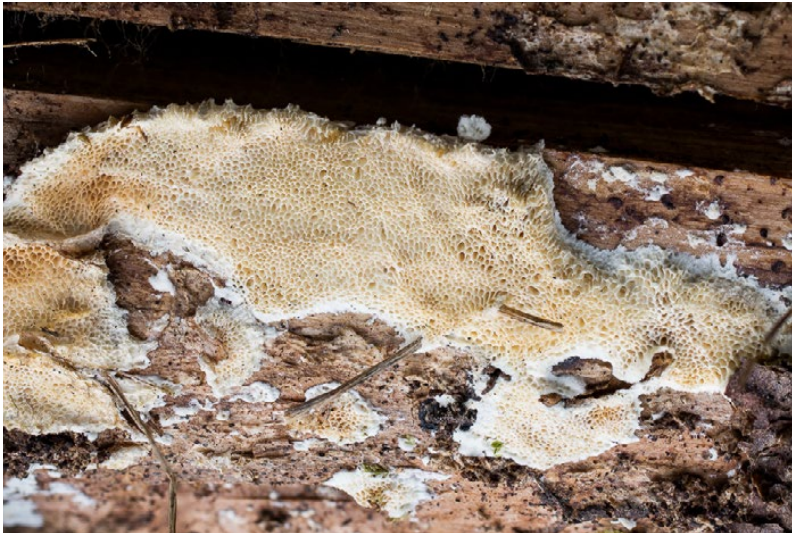

Figure 5. Antrodiella parasitica in Iisalmi (O. Ryhänen 1/9920). Photo: Ossi Ryhänen.

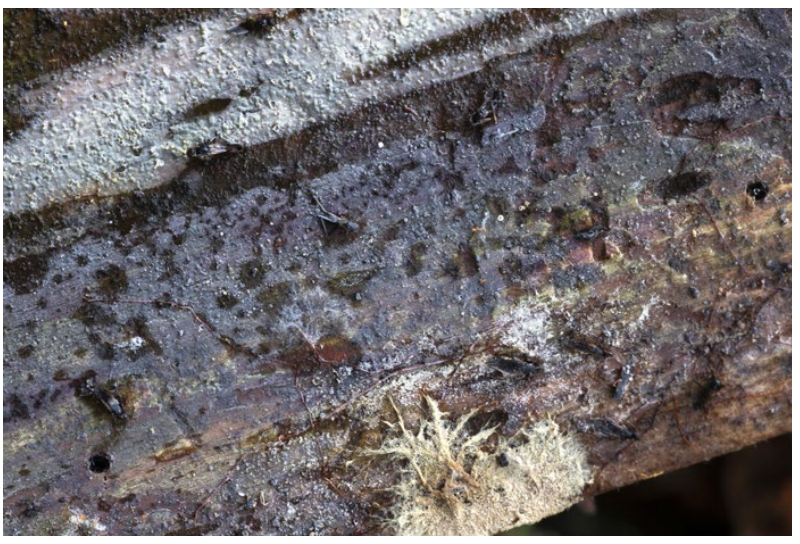

Figure 6. Aphanobasidium subnitens in Kuhmo (TH 20200029). Photo: Teppo Helo.

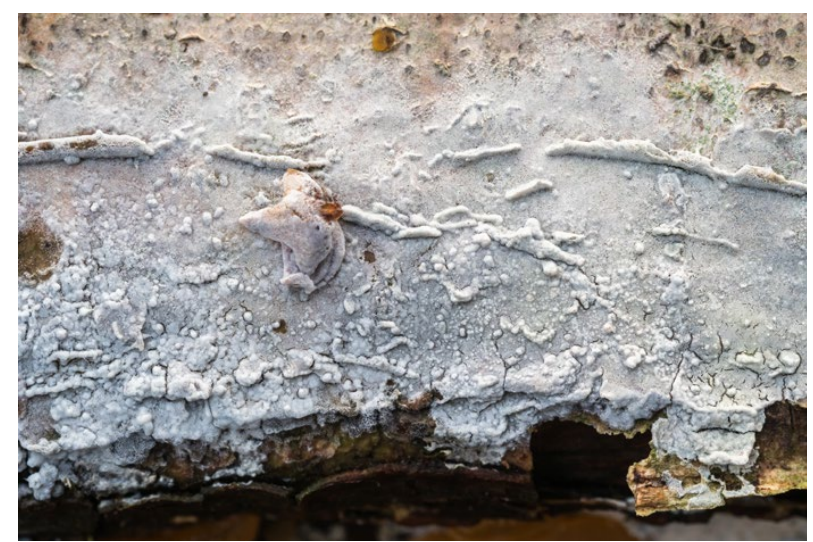

Figure 7. Athelopsis glaucina in Raasepori (JP4241). Photo: Jorma Pennanen.

$4 \mathrm{~cm}$, decay stage 4) in a Corylus avellana dominated herb-rich forest, 31 Dec. 2020, leg. \& det. JP 4241 (H), conf. HK.

\section{Biatoropsis usnearum Räsänen}

(Fig. 8)

Distribution. New to 3 b (Fig. 1).

Specimen examined. Ostrobottnia kajanensis, Kuhmo, Riihivaara, UCS 7127522:3654462, on Usnea glabrescens growing on a living Salix caprea (diam. $35 \mathrm{~cm}$ ) in a very old Picea abies dominated paludified herb-rich heath forest, 26 Aug. 2017, leg. $\&$ det JJ 1101 (OULU). We are aware that there is also an older unpublished museum specimen (KUO) which was collected on the border of zones $3 \mathrm{a}$ and $3 \mathrm{~b}$. Because that specimen lacks 
exact coordinates, we decided to publish our more accurate JJ 1101 record.

\section{Botryobasidium ellipsosporum Holubová-Jechová}

Notes. $4^{\text {th }}$ record in Finland; previous records: Lammi (2a), Puolanka (3b) and Sotkamo (3b) (Kotiranta et al. 2009; Kunttu et al. 2018, 2019). The specimen was an anamorph as were the three previous Finnish collections.

Specimen examined. Ostrobottnia kajanensis, Kuhmo, Elimyssalo, UCS 7129643:3664595, on a fallen trunk of Pinus sylvestris (diam. 25, decay stage 2, kelo tree) with Tulasnella deliquescens and Tylospora fibrillosa in an old pine-dominated sub-xeric heath forest, 9 Oct. 2020, leg. \& det. TH 20200039 (OULU).

Botryobasidium intertextum (Schwein.) Jülich \& Stalpers

Distribution. New to 3a (Fig. 1).

Specimen examined. Ostrobottnia ouluensis, Utajärvi, Hanganvaara, Hanganhete, UCS 7220917:3490636, on a fallen trunk of Alnus incana, in a spruce-dominated herb-rich forest next to a stream running from a spring, 14 July 2018, leg. \& det. AM 74b-18 (OULU), conf. MK.

Brevicellicium olivascens (Bres.) K.H. Larss. \& Hjortstam Distribution. New to 3a (Fig. 1).

Specimen examined. Ostrobottnia ouluensis, Oulu, Kontinkangas, UCS 7212832:3429839, on a fallen trunk of Populus trem$u l a$, in an aspen-dominated herb-rich heath forest adjacent to a road, 12 Oct. 2019, leg. \& det. AM 140 (OULU), conf. MK.

Byssocorticium efibulatum Hjortstam \& Ryvarden

(Fig. 9)

Notes. $2^{\text {nd }}$ record in Finland; previous record: Raasepori (1b) (Kunttu et al. 2012). Not Evaluated.

Specimen examined. Nylandia, Helsinki, Annala, UCS 6679:3387, on pieces of oak branches that were half-buried in the mulch of a mesic herb-rich forest with Quercus robur, Acer platanoides and Pinus sylvestris, 1 May 2020 and 10 Oct. 2020, leg. \& det. PV $1814(\mathrm{H})$ and PV 2554.

Caudicicola gracilis Kotir., Kulju \& Miettinen

(Fig. 10)

Notes. This is the second global record; the first record was reported in Finland, Pyhäjärvi (3a), where basidiomata were discovered growing on six Picea abies or coniferous stumps quite close to each other in a spruce-dominated swamp forest (Kotiranta et al. 2017). Data Deficient.

Specimen examined. Ostrobottnia ouluensis, Oulu, Holtinkylä, Sammakkokangas, UCS 7213406:3440431, on a sawn block of Betula sp. leaning against a tree in a moist spruce-dominated drained and transformed mire, 28 Sept. 2020, leg. \& det. P. Jokikokko (OULU, H), conf. MK. This finding is described in more detail in Jokikokko (2020).

Ceraceomyces borealis (Romell) J. Erikss. \& Ryvarden

Distribution. New to $2 b$ (Fig. 1).

Specimen examined. Savonia borealis, Lapinlahti, Koirniemi, 7033:3548, on a log of Alnus incana (diam. $10 \mathrm{~cm}$, decay stage 2), in a log pile, 12 Aug. 2020, leg. \& det. PV 2091 (H).

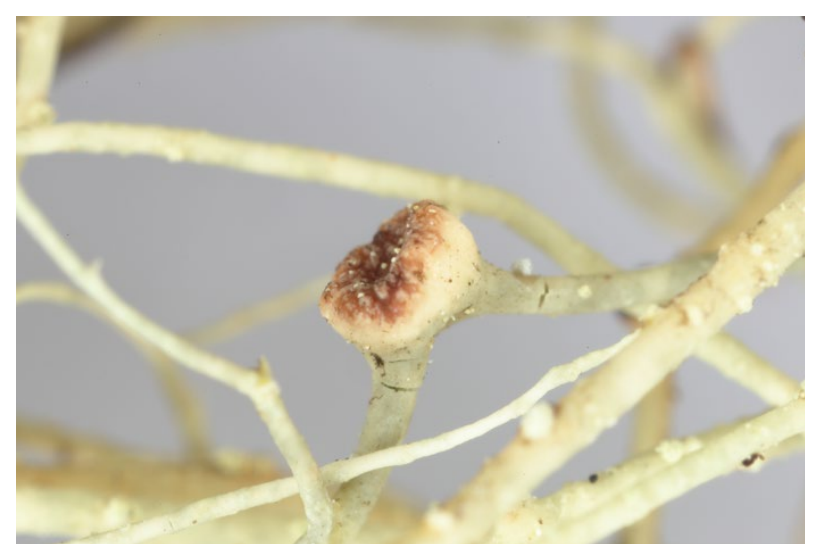

Figure 8. Biatoropsis usnearum in Kuhmo (JJ 1101). Photo: Jari Julkunen.

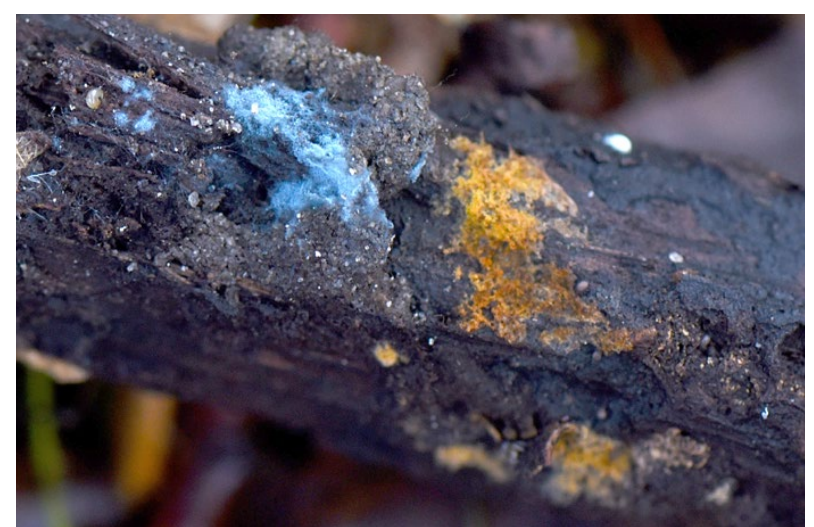

Figure 9. Byssocorticium efibulatum in Helsinki (PV 2554). Photo: Pyry Veteli.

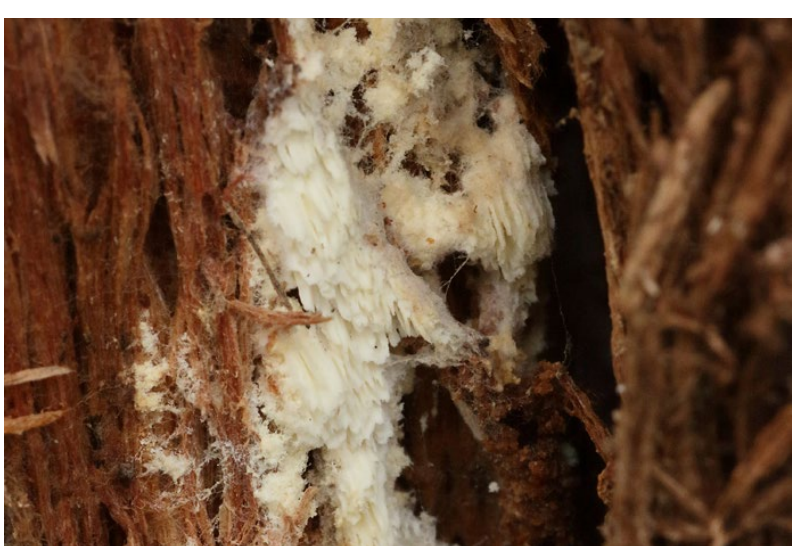

Figure 10. Caudicicola gracilis in Oulu. Photo: Pauli Jokikokko.

\section{Ceraceomyces microsporus K.H. Larsson}

Distribution. New to 3c (Fig. 1).

Specimen examined. Ostrobottnia ultima, Tervola, Pisavaara Strict Nature Reserve, Vähäloma, UCS 7352908:3413439, on a decorticated fallen trunk of Pinus sylvestris (diam. $15 \mathrm{~cm}$, decay stage 2) in an old stony mesic heath forest, 4 Oct. 2020, leg. \& det. MK 61/20 (OULU).

Ceriporia bresadolae (Bourdot \& Galzin) Bondartsev \& Singer

Distribution. New to 1 b (Fig. 1).

Notes. $4^{\text {th }}$ record in Finland; previous records: Muhos (3a), Oulu (3a) and Inari (4c) (Kunttu et al. 2016). 
Specimen examined. Nylandia, Helsinki, Kumpula, Kymintien metsä, UCS 6679:3387, on trunk of dead standing Pinus sylvestris (diam. $28 \mathrm{~cm}$, decay stage 2), rocky forest dominated by Pinus sylvestris, 14.11.2020, leg. \& det. PV $2630(\mathrm{H})$.

\section{Clavaria greletii Boud.}

(Fig. 11)

Notes. $9^{\text {th }}$ record in Finland; previous records: Ikaalinen (2a), Pälkäne (2a), Lappeenranta (2b), Pieksämäki (2b), Kajaani (3b; two sites), Sotkamo (3b), and Keminmaa (3c) (Kotiranta et al. 2009; Kunttu et al. 2016).

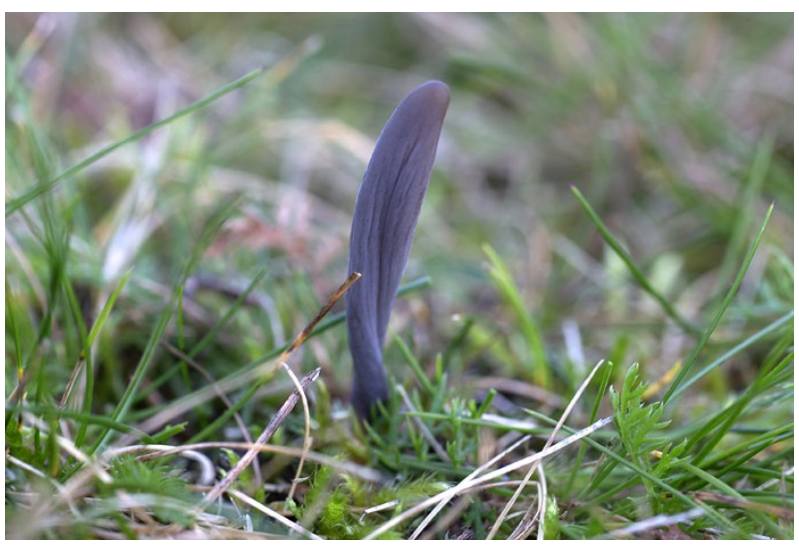

Figure 11. Clavaria greletii in Kuhmo (TH 20200043). Photo: Teppo Helo.

Specimen examined. Ostrobottnia kajanensis, Kuhmo, Tönölä, UCS 711606:362590, in grassland next to road, 7 Oct. 2020, leg. \& det. TH 20200043 (OULU).

Colacogloea peniophorae (Bourdot \& Galzin) Oberw., R. Bauer \& Bandoni

Distribution. New to $4 \mathrm{~b}$ (Fig. 1).

Specimen examined. Ostrobottnia ultima, Rovaniemi, Vanttauskoski, 7371:3488, on fallen Pinus sylvestris trunk (diam. $35 \mathrm{~cm}$, decay stage 5a), leg. PV 2479, det. V. Spirin.

Conferticium ravum (Burt) Ginns \& Freeman

Notes. $9^{\text {th }}$ record in Finland; previous records: Luhanka (2b), Korpilahti (2b), Sulkava (2b), Suonenjoki (2b), Äänekoski (2b), Konnevesi (2b), Viitasaari (2b), and Rovaniemi (3c) (Kotiranta unpubl., Kunttu et al. 2019, 2020). Vulnerable.

Specimen examined. Ostrobottnia ultima, Rovaniemi, Hirvas, UCS 7374:3427, crown branches of a fallen Populus tremula (diam. $30 \mathrm{~cm}$, decay stage 2), in association with Inonotus rheades, Phellinus tremulae, Trametes ochracea and Trichaptum abietinum, in a somewhat paludified depression inside a mesic heath forest dominated by Pinus sylvestris, 21 Sept. 2020, leg. \& det. PV $2456(\mathrm{H})$.

Corticium boreoroseum Boidin \& Lanq.

(Fig. 12)

Distribution. New to 4a (Fig. 1).

Specimen examined. Regio kuusamoënsis, Kuusamo, Repokallio, UCS 7285176:3623085, on a fallen Picea abies trunk (decay stage 1-2), in a very old pine- and spruce-dominated mesic heath forest - subxeric heath forest, 6 Oct. 2018, leg. \& det, JJ1274 (OULU), conf. MK.

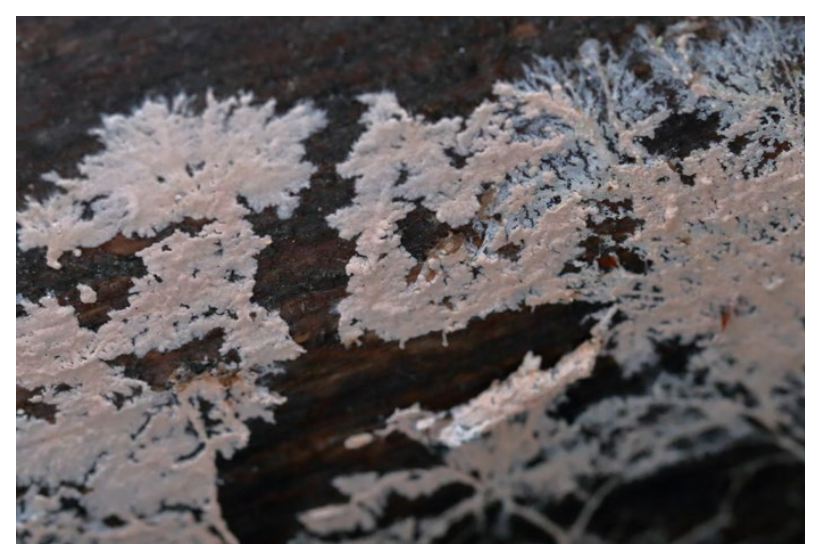

Figure 12. Corticium boreoroseum in Kuusamo (JJ 1274). Photo: Jari Julkunen.

\section{Crustoderma longicystidiatum (Litsch.) Nakasone}

Notes. $2^{\text {nd }}-3^{\text {rd }}$ records in Finland; previous records: Miehikkälä (2a) (Kotiranta et al. 2009). The specimens are conspecific according to ITS-sequences. Notably, both specimens are from coniferous substrate exposed to prolonged cool and humid or wet conditions which may have been the case with the Miehikkälä-collection as well (the habitat was timber sawmill located near rapids). Whether the same holds for the species type, warrants a more thorough taxonomic treatment in the future. Critically Endangered.

Specimens examined. Tavastia borealis, Vuoritsalo, UCS 6900:3438, tree-shaded yard with long grass, on a cut piece of wood (diam. $9 \mathrm{~cm}$, decay stage 3), in association with Hyphoderma praetermissum, Scopuloides hydnoides, 20 Jul. 2007, leg. \& det. OM 11790.1 (H, specimen sequenced); Savonia borealis, Lapinlahti, Koirniemi, UCS 7034:3547, on a log of a Pinus sylvestris kelo tree (diam. $30 \mathrm{~cm}$, decay 4), washed-up by a flooding river. The $\log$ was used in guiding structures in rafting operations until the 1956 waterway conversion to hydropower use. Found in association with Hyphoderma praetermissum and Botryobasidium sp., 24 Jul. 2019 leg. PV 1094 \& det. PV \& HK (H, specimen sequenced).

Crustoderma triste (Litsch. \& Lundell) Duhem

Notes. $6^{\text {th }}$ record in Finland; previous records: Jyväskylä (2b), Saarijärvi (3a), Lieksa (3b), Kuusamo (4a), and Inari (4c) (Kotiranta et al. 2009; FinBIF 2021c).

Specimen examined. Ostrobottnia kajanensis, Kuhmo, Ulvinsalo, UCS 7103310:3665104, on fallen trunk of Pinus sylvestris (diam. 20, decay stage 4, kelo tree) with Paullicorticium sp. in a very old pine-dominated sub-xeric heath forest, 3 Oct. 2020, leg. TH 20200022 (OULU, H). det. MK.

\section{Dendrothele amygdalispora Hjortstam}

(Fig. 14)

Distribution. New to 3b (Fig. 1).

Specimen examined. Ostrobottnia kajanensis, Kajaani, Old cemetery, UCS 71262:35347, on trunk and branch of Salix fragilis 'Bullata' in a park-like cemetery, 29 Oct. 2020, leg. \& det. TH 20200044 (OULU).

Exidia candida var. cartilaginea (S. Lundell \& Neuhoff) Spirin \& V. Malysheva

Distribution. New to 3c (Fig. 1). 


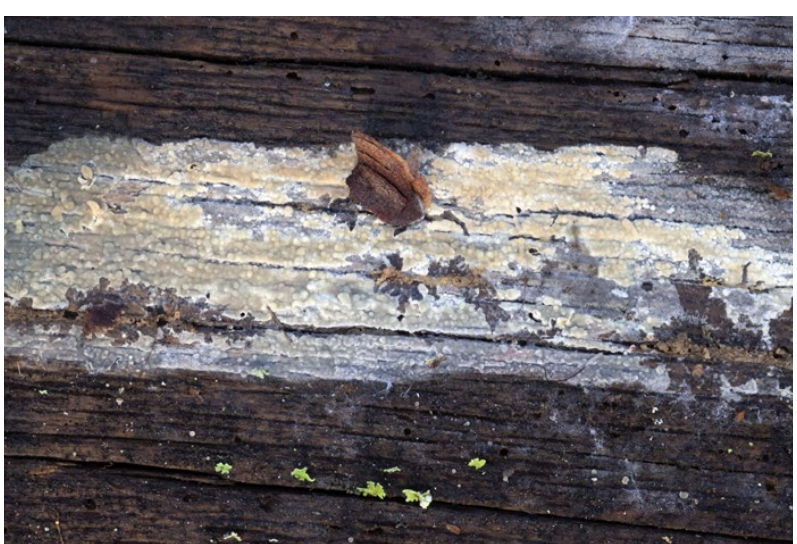

Figure 13. Crustoderma triste in Kuhmo (TH 20200022) with Paullicorticium sp. Photo: Teppo Helo.

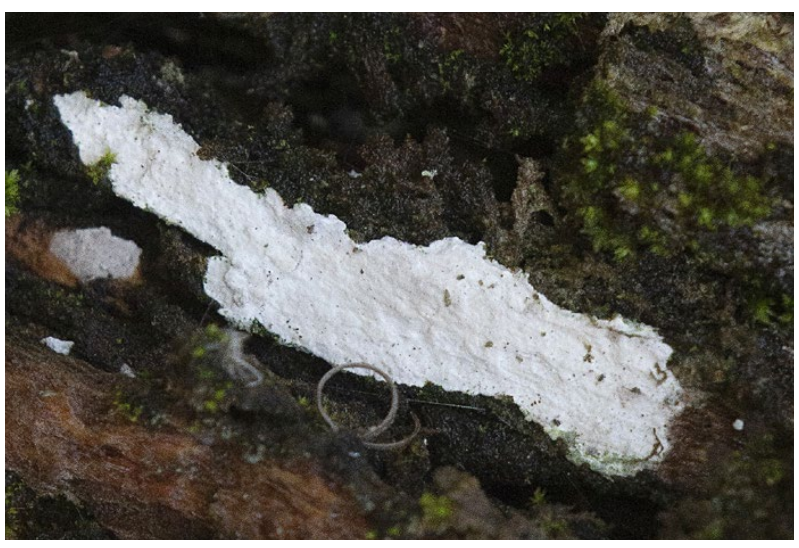

Figure 14. Dendrothele amygdalispora in Kajaani (TH 20200044). Photo: Teppo Helo.

Specimen examined. Ostrobottnia ultima, Tervola, Pisavaara Strict Nature Reserve, Vähäloma, UCS 7352766:3413304, on a corticated fallen trunk of Betula sp. (diam. $10 \mathrm{~cm}$, decay stage 1) in an old spruce-dominated mesic heath forest, 4 Oct. 2020, leg. \& det. MK 50/20 (OULU).

\section{Gloiothele lactescens (Berk.) Hjortstam}

Notes. $2^{\text {nd }}-3^{\text {rd }}$ records in Finland: previous record: a park in Helsinki (Kotiranta et al. 2009). Near Threatened.

Specimen examined. Nylandia, Helsinki, Sibelius Park, UCS 667611:3844, on a decorticated trunk and a stump of Tilia sp. (diam. $35 \mathrm{~cm}$, decay stage 4) plus on a board, 23 Sept. 2010 and yearly till 30 Oct. 2014, but not anymore on the stump 2016 when the trunk had been removed, leg. \& det. HK 22720, 25409, 26213, 26214, 26215, 26501 (H); Helsinki, Kustaankartano, UCS 6681:3386, on fallen Salix sp stem (diam. $10 \mathrm{~cm}$, decay stage 3), former agricultural land, now herb-rich forest with high amounts of coarse dead wood, stand dominated by Salix caprea and Ulmus glabra, 3.10.2019, leg. \& det. PV 1364 (H), conf. HK.

Hapalopilus ribicola (P. Karst.) Spirin \& Miettinen

Distribution. New to 3a (Fig. 1).

Specimen examined. Ostrobottnia ouluensis, Liminka, Kirkonkylä, UCS 7191178:3424547, on branches of Ribes nigrum in the yard, 17 May 2020, leg. \& det. P. Jokikokko (OULU), conf. MK.

Helicogloea dryina Spirin \& Miettinen

(Fig. 15)

Distribution. New to $3 \mathrm{a}-\mathrm{b}$ (Fig. 1).

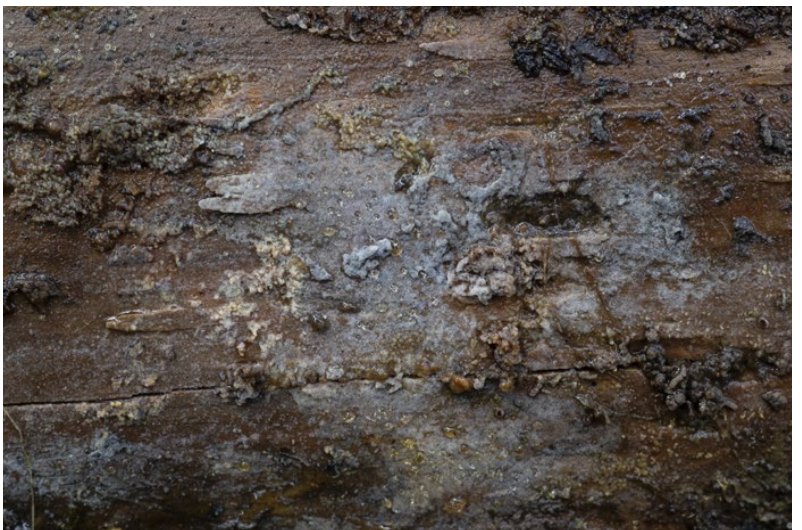

Figure 15. Helicogloea dryina in Sotkamo (TH 20200010). Photo: Teppo Helo.

Notes. $3^{\text {rd }}-7^{\text {th }}$ records in Finland; previous records: Helsinki (2a) and Vantaa (2a) (Malysheva et al. 2020; FinBIF 2021c).

Specimen examined. Ostrobottnia ouluensis, Oulu, Metsokangas, UCS 7207308:3432036, on a partly decorticated fallen trunk of Pinus sylvestris (diam. $15 \mathrm{~cm}$, decay stage 1) in a wet forested old field, 6 Nov. 2020, leg. \& det. MK 92/20 (OULU, H), conf. V. Spirin; Ostrobottnia kajanensis, Sotkamo, Losonvaara, UCS 7106773:3545205, on a fallen trunk of Picea abies (diam. $30 \mathrm{~cm}$, decay stage 3), in an old spruce-dominated mesic heath forest, 30 Sept. 2020, leg. \& det. TH 20200045 (OULU); Sotkamo, Talvivaara, UCS 7093231:3557660, on a fallen trunk of Picea abies (diam. $15 \mathrm{~cm}$, decay stage 3), in an old spruce-dominated mesic heath forest, 24 Sept. 2020, leg. \& det. TH 20200010 (OULU), conf. V. Spirin; Kuhmo, Elimyssalo, UCS 71265:36593, on a fallen trunk of Picea abies (diam. $10 \mathrm{~cm}$, decay stage 3), in an old spruce-dominated mesic heath forest, 6 Oct. 2020, leg. TH 20200047 (OULU), det. MK; Kuhmo, Ulvinsalo, UCS 7103386:3664731, on a fallen trunk of Picea abies (diam. $45 \mathrm{~cm}$, decay stage 4 ), in a very old thin-peated spruce mire, 4 Oct. 2020, leg. \& det. TH 20200046 (OULU)

Hyalodon piceicola (Kühner ex Bourdot) Malysheva \& Spirin

(Fig. 16)

Distribution. New to $1 \mathrm{~b}$ (Fig. 1).

Specimen examined. Nylandia, Raasepori, Fiskars, Risslaån, UCS 6672561:308094, on a piece of wood of Picea abies (diam. $10 \mathrm{~cm}$, decay stage 4) in a brookside herb-rich forest, 21 Jan. 2020, leg. \& det. JP 4264 (H).

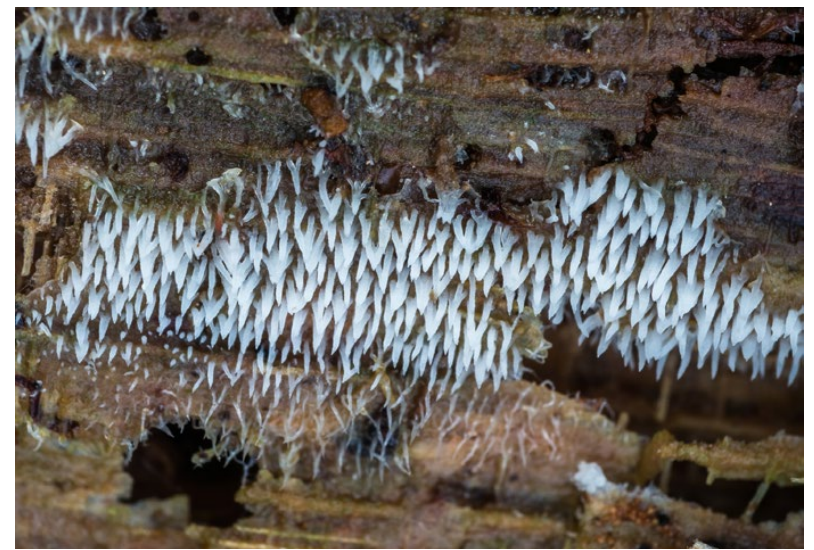

Figure 16. Hyalodon piceicola in Raasepori (JP 4264). Photo: Jorma Pennanen. 


\section{Hyphoderma lapponicum (Litsch.) Ryvarden}

Distribution. New to Finland and hence new to $4 \mathrm{~d}$ (Fig. 1).

Specimen examined. Lapponica enontekiensis, Enontekiö, Jogasjávri N, Doskaljohka W, UCS 768596:328070, on dead trunk or branch of Betula pubescens subsp. czerepanovii, in a moist to dry mountain birch forest, 27 Jul. 2020, leg. H. Väre $25318(\mathrm{H})$, det. MK, conf. HK.

\section{Hypochnicium subrigescens Boidin}

(Fig. 17)

Distribution. New to 3 b (Fig. 1).

Specimen examined. Ostrobottnia kajanensis, Kuhmo, Elimyssalo, UCS 7126464:3660017, on a fallen branch of Populus tremula (diam. $30 \mathrm{~cm}$, decay stage 2), in an old dwarf shrub spruce mire, 6 Oct. 2020, leg. TH 20200049 (OULU), det. MK; Kuhmo, Ulvinsalo, UCS 7103767:3665034, on a fallen trunk of Populus tremula (diam. $30 \mathrm{~cm}$, decay stage 4), in a very old spruce-dominated mesic heath forest, 2 Oct. 2020, leg. \& det. TH 20200048 (OULU).

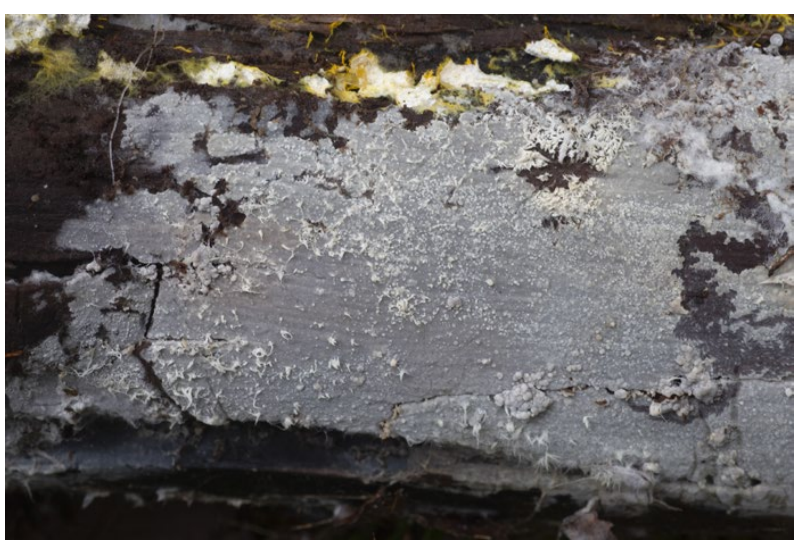

Figure 17. Hypochnicium subrigescens in Kuhmo (TH 20200049). Photo: Teppo Helo.

\section{Jaapia argillacea Bres.}

(Fig. 18)

Distribution. New to $3 b$ (Fig. 1).

Specimen examined. Ostrobottnia kajanensis, Kuhmo, Ulvinsalo, UCS 7103386:3664703, on a fallen trunk of Picea abies (diam. $40 \mathrm{~cm}$, decay stage 4) with Jaapia ochroleuca and Dichostereum boreale in a very old dwarf shrub spruce mire, 4 Oct. 2020, leg. \& det. TH 20200050 (OULU), conf. MK.

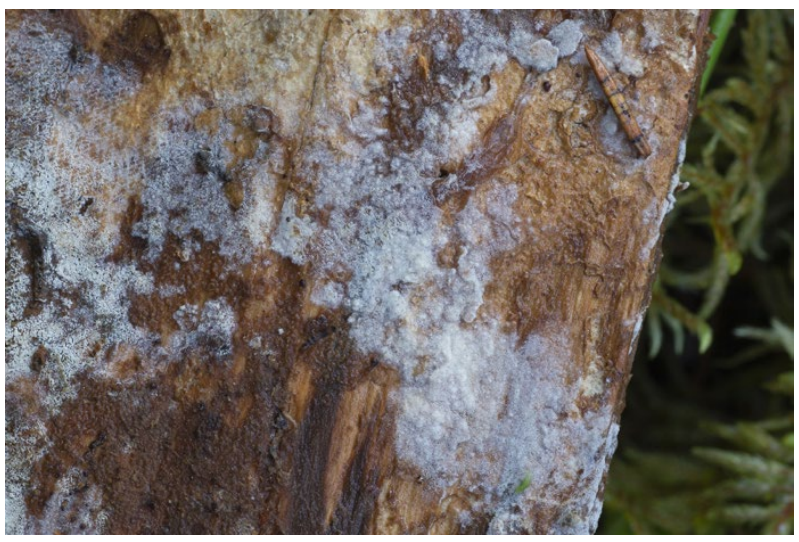

Figure 18. Jaapia argillacea in Kuhmo (TH 20200050) with Dichostereum boreale. Photo: Teppo Helo.

\section{Leptosporomyces fuscostratus (Burt) Hjortstam}

Distribution. New to 3a (Fig. 1).

Specimen examined. Ostrobottnia ouluensis, Oulu, Metsokangas, UCS 7207125:3432114, on a decorticated stump of Picea abies (diam. $23 \mathrm{~cm}$, decay stage 4) in a mesic heath forest, 4 Nov. 2020, leg. \& det. MK 89/20 (OULU), conf. HK.

\section{Lindtneria panphyliensis Bernicchia}

(Fig. 19)

Notes. $5^{\text {th }}-7^{\text {th }}$ records in Finland; previous records: four sites in Helsinki (Miettinen 2012).

Specimens examined. Nylandia, Helsinki, Kustaankartano, UCS 6681:3386, on a thin stem of a fallen dead Acer platanoides (diam. $8 \mathrm{~cm}$, decay stage 2), pressed to the organic/clay soil, herb-rich site, mixed stand of old conifers with Acer, Betula,

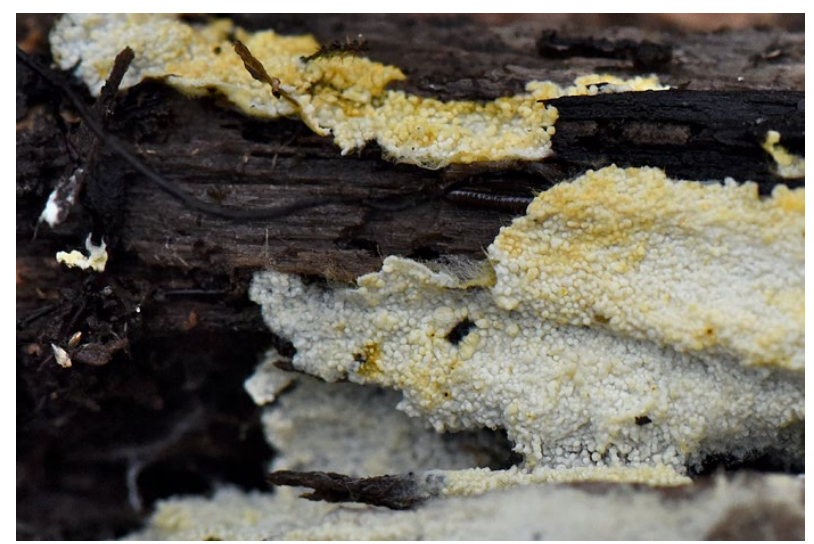

Figure 19. Lindtneria panphyliensis in Helsinki (PV 2589). Photo: Pyry Veteli.

Sorbus, Salix and Alnus middle- and understory, 23 Oct. 2019, leg. PV 1524 (H); Helsinki, Patomäenpuisto, UCS 6682:3387, far decayed piece of deciduous wood, pressed to organic/clay soil, herb-rich site dominated by Picea abies, Betula pendula and Acer platanoides, 07 Nov. 2020, leg. S. Pousi, P. Tolvanen, PV 2607 (H), det. PV; Helsinki, Taivaskallio, UCS 6680:3386, on trunk of fallen Sorbus aucuparia, pressed to rich organic soil, nearby Acer platanoides, Sorbus aucuparia and Pinus sylvestris, 30 Oct. 2020, leg. PV 2589 (H).

Litschauerella clematitis (Bourdot \& Galzin) J. Erikss. \& Ryvarden

(Fig. 20)

Distribution. New to 3 b (Fig. 1).

Notes. $5^{\text {th }}$ record in Finland; previous records: Tammisaari (1b), Helsinki (1b), Jyväskylä (2b), and Utsjoki (4d) (Kotiranta et al. 2009; Kotiranta \& Shiryaev 2013; Kunttu et al. 2018).

Specimen examined. Ostrobottnia kajanensis, Sotkamo, Losonvaara, UCS 7107287:3545992, on a fallen trunk of Juniperus communis (diam. $6 \mathrm{~cm}$, decay stage 3), in a middle-aged spruce-dominated mesic heath forest, 28 Sept. 2020, leg. \& det. TH 20200051 (OULU).

Luellia recondita (H.S. Jacks.) K.H. Larsson \& Hjortstam

(Fig. 21)

Distribution. New to 3b (Fig. 1).

Notes. $8^{\text {th }}$ record in Finland; previous records: Eckerö (1a), Helsinki (1b), Tammisaari (1b), Kemiönsaari (1b), 


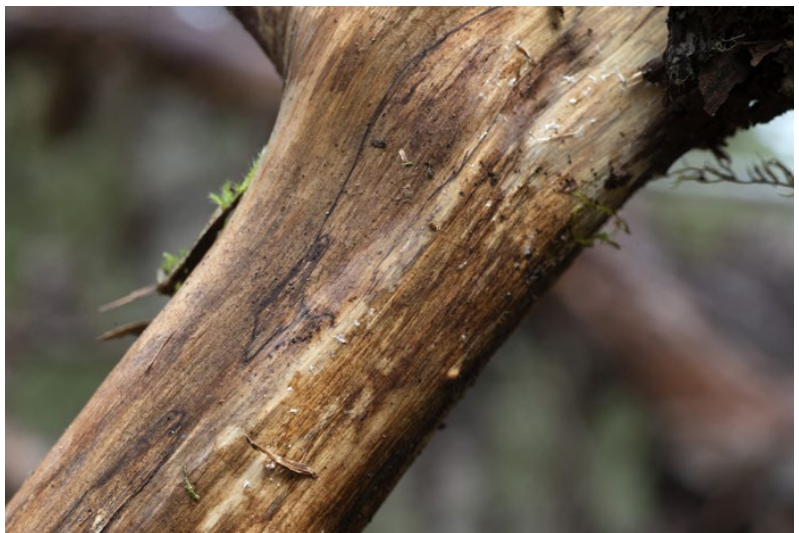

Figure 20. Litschauerella clematitis in Sotkamo (TH 20200051). Photo: Teppo Helo.

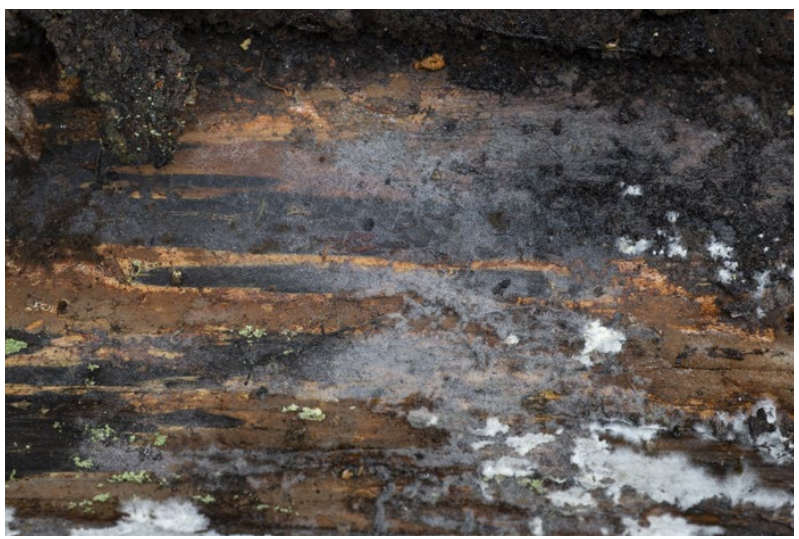

Figure 21. Luellia recondita in Kuhmo (TH 2020052) with Tulasnella pallida and Sistotrema autumnale. Photo: Teppo Helo.

Hamina (2a), Jyväskylä (2b), and Rovaniemi (3c) (Kotiranta et al. 2009; Kunttu et al. 2012, 2015, 2016, 2018).

Specimen examined. Ostrobottnia kajanensis, Kuhmo, Elimyssalo, UCS 71265:36593, on a fallen trunk of Picea abies (diam. $10 \mathrm{~cm}$, decay stage 1) with Tulasnella pallida and Sistotrema autumnale in an old spruce-dominated mesic heath forest, 6 Oct. 2020, leg. TH 20200052 (OULU), det. MK.

Mycostilla vermiformis (Berk. \& Broome) Spirin \& Malysheva

(Fig. 22)

Distribution. New to Finland, and hence new to $1 \mathrm{~b}, 3 \mathrm{~b}$ (Fig. 1).

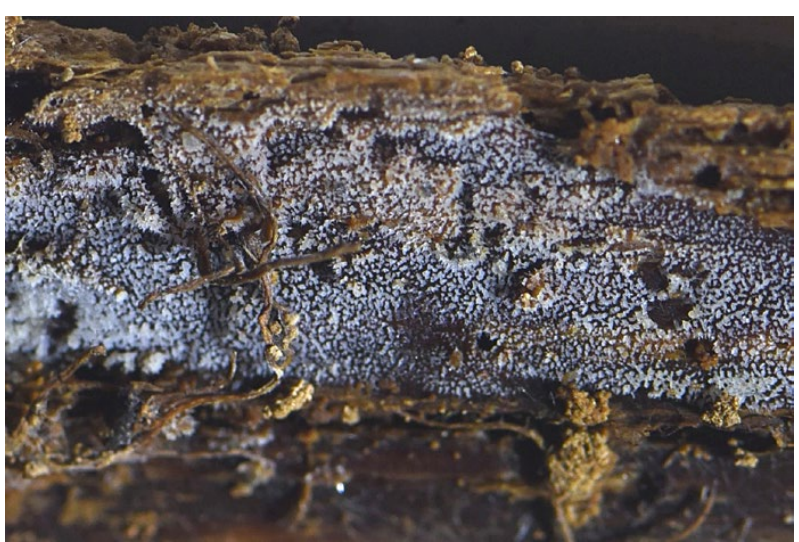

Figure 22. Mycostilla vermiformis in Helsinki (PV 1874). Photo: Pyry Veteli.
Specimens examined. Nylandia, Helsinki, Pirkkola, UCS 6681:3384, on a log of Picea abies (diam. $15 \mathrm{~cm}$, decay stage 3), mesic heath forest dominated by Pinus sylvestris, 20.5.2020, leg. \& det. PV 1874 (H), conf. V. Spirin; Ostrobottnia kajanensis, Sotkamo, Talvivaara, UCS 7092462:3558456, on fallen trunk of Picea abies (diam. $45 \mathrm{~cm}$, decay stage 3), in an old spruce-dominated mesic heath forest, 20 Aug. 2020, leg. TH 20200006 (OULU, H), det. V. Spirin.

\section{Oligoporus romellii (Pieri \& Rivoire)}

Distribution. New to 4 a (Fig. 1).

Specimen examined. Regio kuusamoënsis, Kuusamo, Penttilänvaara W, Kontiojoki S, UCS 7299549:3589596, on a fallen trunk of Picea abies (diam. $40 \mathrm{~cm}$, decay stage 2/3), in a sprucepine mire - dwarf shrub pine mire, 7 Oct. 2005, leg. T. Laitinen 732 (OULU), det. MK.

\section{Paullicorticium ansatum Liberta}

(Fig. 23)

Distribution. New to 3 b (Fig. 1).

Notes. $3^{\text {rd }}$ record in Finland; previous records: Lammi (2a) and Padasjoki (2a) (Kotiranta et al. 2009). Near Threatened.

Specimen examined. Ostrobottnia kajanensis, Kuhmo, Elimyssalo, UCS 7130666:3662462, on a fallen trunk of Pinus sylvestris (diam. $30 \mathrm{~cm}$, decay stage 3 , kelo tree), in an old pine-dominated sub-xeric heath forest, 12 Oct. 2020, leg. \& det. TH 20200053 (OULU), conf. MK.

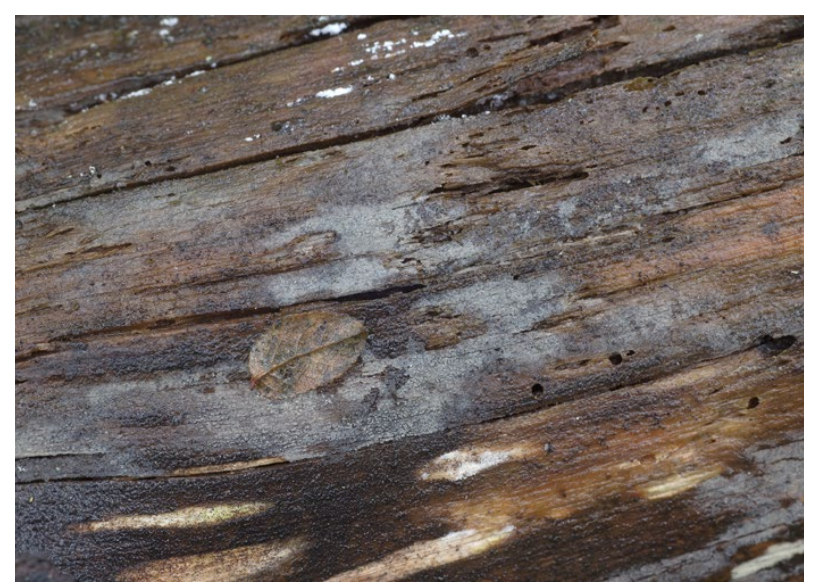

Figure 23. Paullicorticium ansatum in Kuhmo (TH 20200053). Photo: Teppo Helo.

\section{Peniophora cinerea (Pers.: Fr.) W.B Cooke}

Distribution. New to 3b (Fig. 1).

Specimen examined. Ostrobottnia kajanensis, Kuhmo, Elimyssalo, UCS 71285:36651, on a fallen trunk of Populus tremula (diam. $10 \mathrm{~cm}$, decay stage 3 ), in an old pine-dominated sub-xeric heath forest, 10 Oct. 2020, leg. \& det. TH 20200056 (OULU), conf. MK.

\section{Peniophora nuda (Fr.) Bres.}

(Fig. 24)

Distribution. New to 3 b (Fig. 1).

Specimen examined. Ostrobottnia kajanensis, Kuhmo, Elimyssalo, UCS 71295:36645, on a fallen trunk of Betula sp. (diam. $30 \mathrm{~cm}$, decay stage 2), in an old dwarf shrub spruce mire, 9 Oct. 2020, leg. \& det. TH 20200057 (OULU), conf. MK. 


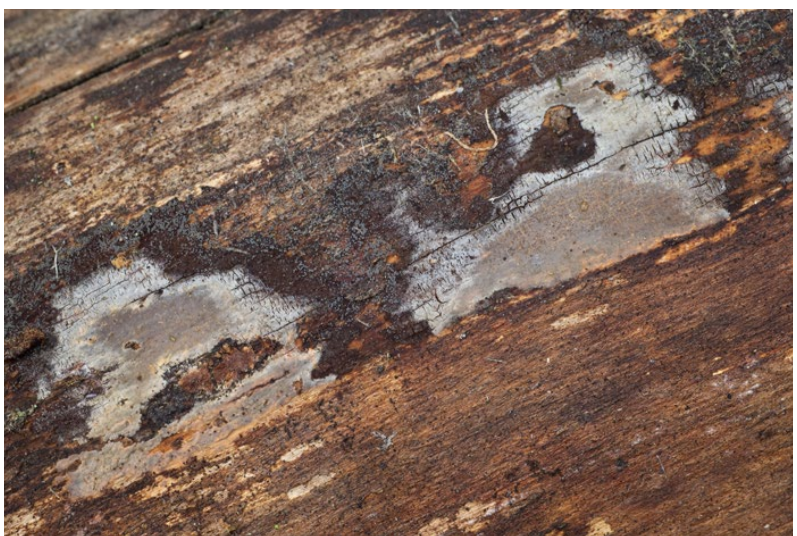

Figure 24. Peniophora nuda in Kuhmo (TH 20200057). Photo: Teppo Helo.

\section{Peniophora pini (Schleich.: Fr.) Boidin}

(Fig. 25)

Distribution. New to $3 \mathrm{~b}$ (Fig. 1).

Specimen examined. Ostrobottnia kajanensis, Sotkamo, Losonvaara, UCS 71072:35460, on a fallen branch of Pinus sylvestris (diam. $7 \mathrm{~cm}$, decay stage 1), in a middle-aged spruce-dominated mesic heath forest, 28 Sept. 2020, leg. \& det. TH 20200058 (OULU), conf. MK.

Peniophora quercina (Pers.: Fr.) W. B. Cooke

(Fig. 26)

Notes. $2^{\text {nd }}-5^{\text {th }}$ records in Finland; the previous: Karjaa (1b) (Kotiranta et al. 2009). Endangered.

Specimen examined. Regio aboënsis, Perniö, Arpalahti, Kaapinmäki, UCS 6692:3285, on a fallen branch of Quercus robur in an herb-rich forest dominated by old oak trees, 8 Nov. 2004, leg. P. Heinonen \& M.-L. Heinonen (TUR, H); Nylandia, Helsinki, Veräjälaakso, on a recently fallen branch of a large living Quercus robur, 20 Sept. 2014, leg. A. Käppi, det. V. Spirin (H); Helsinki, Koskela, UCS 6680:3387, on a corticated branch of Quercus robur (diam. $0.6 / 1.3 \mathrm{~cm}$, decay stage 2 ) in a cultivated oak park, with Peniophora incarnata and P. nuda, 30 Nov. 2014, leg. \& det. HK (H); Nylandia, Helsinki, Maunulanpuisto, UCS 6681:3384, on Quercus robur, colonized branches (diam. $4 \mathrm{~cm}$, decay 2-3) that had fallen from standing living trees on an oak plantation in agricultural field, 20 Mar. 2020, leg. \& det. PV 1722 (H).

\section{Phellodon secretus Niemelä \& Kinnunen}

Distribution. New to 3c (Fig. 1).

Specimen examined. Ostrobottnia ultima, Rovaniemi, Hirvas, UCS 7374:3427, under a burnt Pinus sylvestris stump (diam. $17 \mathrm{~cm}$, decay stage 2) together with Hydnellum gracilipes, in a managed mesic heath forest dominated by Pinus sylvestris, very small amount of coarse woody debris, with indication of past fires in the soil, 20 Sept. 2020, leg. \& det. PV 2445. Vulnerable.

Phlebia subcretacea (Litsch.) M.B. Christ.

Distribution. New to 3c, 4b (Fig. 1).

Specimens examined. Ostrobottnia ultima, Rovaniemi, Hirvas, UCS 7374:3427, fallen Pinus sylvestris trunk (diam. $37 \mathrm{~cm}$, decay stage 4), in a managed mesic heath forest, open stand with small amount of coarse dead wood 21 Sept. 2020, leg. \& det. PV 2449 (H); Rovaniemi, Vanttauskoski, UCS 7371:3488, on

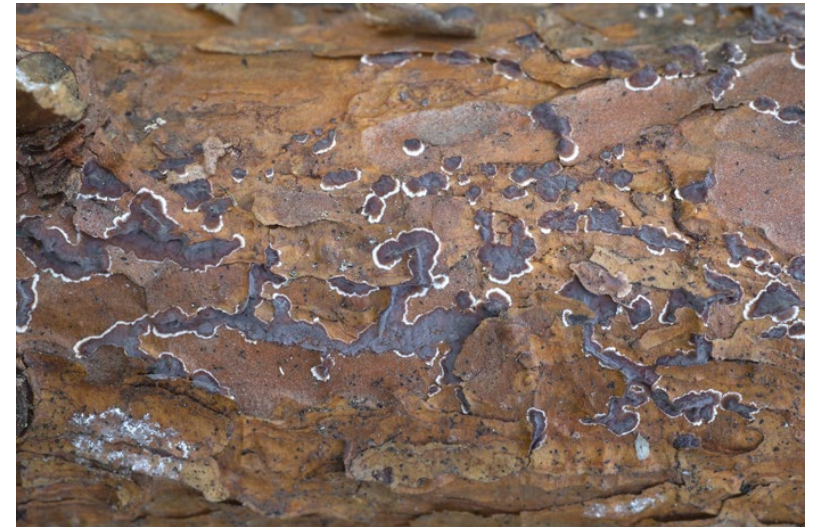

Figure 25. Peniophora pini in Sotkamo (TH 20200058). Photo: Teppo Helo.

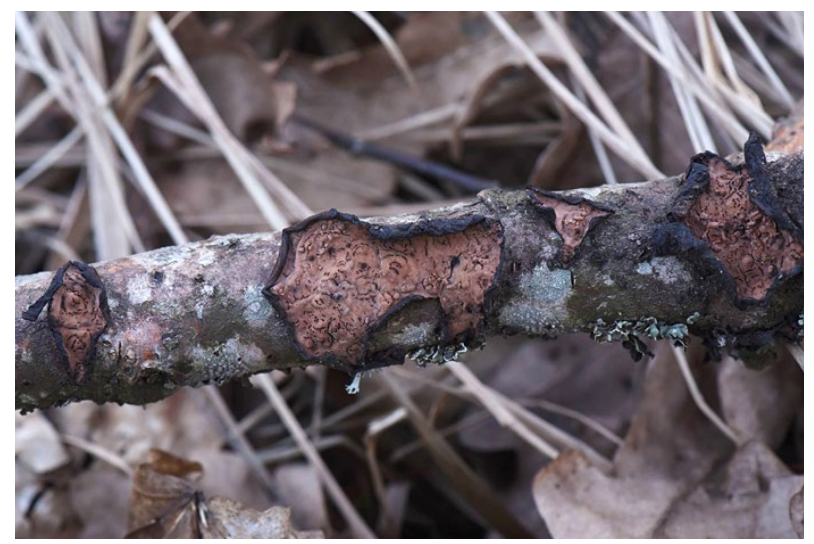

Figure 26. Peniophora quercina in Helsinki (PV 1722). Photo: Pyry Veteli.

a fallen trunk of Pinus sylvestris (diam. $30 \mathrm{~cm}$, decay stage 5b), with Odonticium romellii, mesic and partly paludified heath forest with thick moss cover. Dense stand dominated by Pinus sylvestris mixed with Picea abies and Betula spp., with moderately large amounts of coarse dead wood including kelo pines, 25 Sept. 2020, leg. \& det. PV 2494 (H).

\section{Postia persicina Niemelä \& Y.C. Dai}

(Fig. 27)

Notes. $5^{\text {th }}$ in Finland; previous records: Kuusamo (4a), Saarijärvi (3a), Hyrynsalmi (3b), and Kolari (4b) (Kotiranta et al 2009; Niemelä 2016; Kunttu et al. 2018; FinBIF 2021c). Critically Endangered.

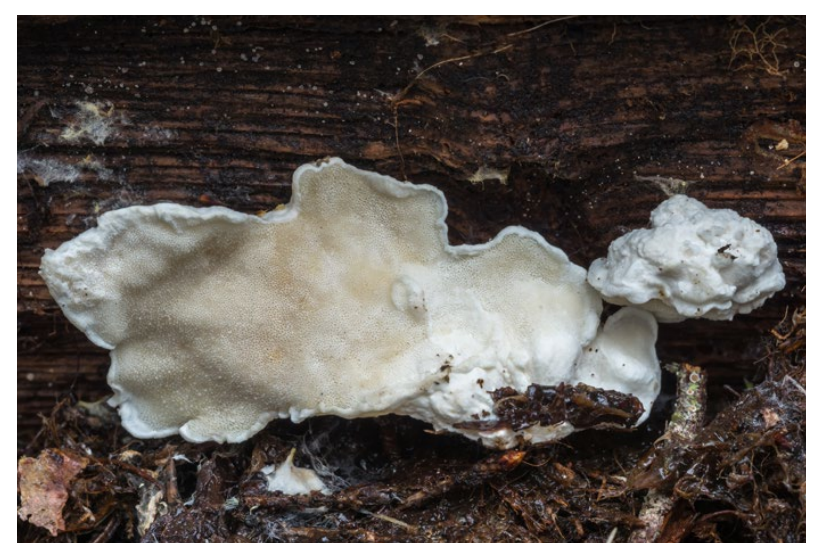

Figure 27. Postia persicina in Kuhmo (JP 3198). Photo: Jorma Pennanen. 
Specimen examined. Ostrobottnia kajanensis, Kuhmo, Ulvinsalo Strict Nature Reserve, UCS 7100248:3665269, on a fallen trunk of Pinus sylvestris (diam. $23 \mathrm{~cm}$, decay stage 3 ) in an old Vaccinum-Myrtillus type pine-dominated mesic heath forest, 12 Sept. 2016, leg. JP 3198 (H), det. OM (specimen sequenced).

'Postia rufescens Spirin \& Miettinen' ined.

Distribution. New to 3a (Fig. 1).

Specimen examined. Satakunta, Ikaalinen, Seitseminen National Park UCS 6876613:3309436, on a fallen trunk of Pinus sylvestris (diam. $20 \mathrm{~cm}$, decay stage 3 ) in a mixed mesic heath forest, 24 Sept. 2019, leg. \& det. JP 4073 (H).

Proterochaete adusta (Burt) Spirin \& V. Malysheva

(Fig. 28)

[Syn. Sebacina adusta Burt, Protodontia oligacantha G.W. Martin, Exidiopsis pallida K. Wells \& Raitv.]

Distribution. New genus and species to Finland and hence new to $2 \mathrm{a}, 3 \mathrm{~b}$ (Fig. 1).

Specimens examined. Ostrobottinia kajanensis, Kuhmo, Riihivaara, UCS 7127339:3654469, on a bark of a fallen and hollow Populus tremula trunk (diam. $40 \mathrm{~cm}$, decay stage 2) in a very old Picea abies dominated thin-peated Vaccinium myrtillus Vaccinium vitis-idaea spruce mire, 26 Aug. 2017, leg. JJ 1062 (OULU, H), det. V. Spirin; Tavastia australis, Hämeenlinna, Härkämäki UCS 6792834:3394898 (probably inaccurate), 23 Sept. 1964, leg V. Hintikka, L. Laine, V. Kujala, L. K. Weresub (H), det. V. Spirin.

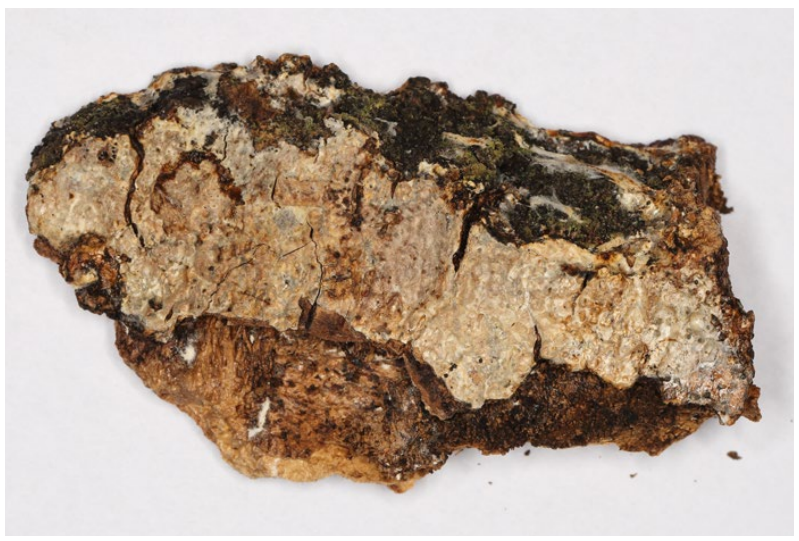

Figure 28. Proterochaete adusta in Kuhmo (JJ 1062). Photo: Jari Julkunen.

\section{Protodontia subgelatinosa (P. Karst.) Pilát （Fig. 29)}

[Syn. Stypella subgelatinosa (P. Karst.) P. Roberts]

Distribution. New to $3 \mathrm{~b}, 4 \mathrm{~d}$ (Fig. 1).

Notes. $7^{\text {th }}-8^{\text {th }}$ records in Finland; previous records: Helsinki (1b; two sites) Tammela (2a), Kangasala (2a), Petäjävesi (2b), Luhanka (2b), and Oulu (3a). (Kotiranta et al. 2009; Kunttu et al. 2012, 2018; Miettinen 2012).

Specimens examined. Savonia borealis, Sonkajärvi, Sonkalahti, UCS 7063:3525, on fallen trunk of Betula pubescens (diam. $25 \mathrm{~cm}$, decay stage 4), on dead Inonotus obliquus basidioma, leg. PV 2108 (H), det.V. Spirin; locus as above, on fallen trunk of Betula pubescens (diam. $15 \mathrm{~cm}$, decay stage 3), with Trechispora cohaerens, leg. PV 2105, det. V. Spirin; Lapponica enontekiensis, Enontekiö, Jogasjávri, Doskaljohka W, UCS

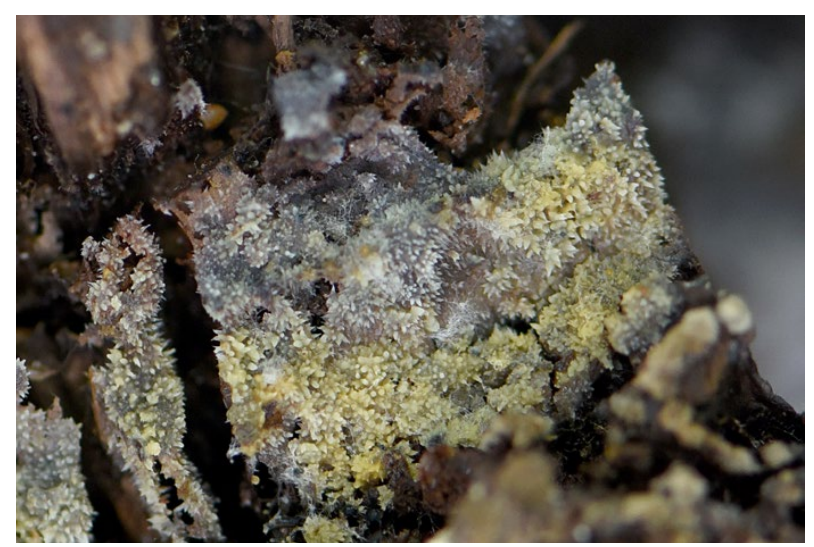

Figure 29. Protodontia subgelatinosa in Sonkajärvi (PV 2108). Photo: Pyry Veteli.

768596:328070, on a dead trunk or branch of Betula nana, in a moist to dry mountain birch forest, 27 Jul. 2020 H. Väre 25321 $(\mathrm{H})$, det. MK, conf. HK.

Protomerulius brachysporus (Luck-Allen) Spirin \& V. Malysheva

(Fig. 30)

Distribution. New to 3 b (Fig. 1).

Notes. $4^{\text {th }}$ record in Finland; previous records: Espoo (1b), Lohja (1b), and Vehmaa (1b) (Spirin et al. 2019a).

Specimen examined. Ostrobottnia kajanensis, Kuhmo, Elimyssalo, UCS 7128815:3665112, on a fallen trunk of Picea abies (diam. $25 \mathrm{~cm}$, decay stage 2), in an old spruce-dominated mesic heath forest, 10 Oct. 2020, leg. \& det. TH 20200036 (OULU, H), conf. MK \& V. Spirin.

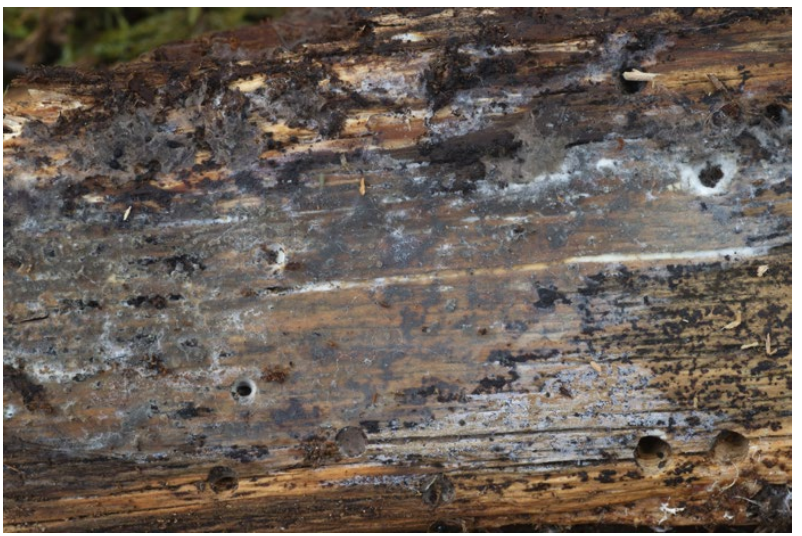

Figure 30. Protomerulius brachysporus in Kuhmo (TH 20200036) with Tulasnella sp. Photo: Teppo Helo.

\section{Pseudotomentella alobata Svantesson}

(Fig. 31)

Distribution. New to Finland and hence new to $3 \mathrm{~b}$ (Fig. 1).

Specimen examined. Ostrobottnia kajanensis, Kajaani, Pöllyvaara, UCS 7126:3535, on fallen trunk of Pinus sylvestris (diam. $12 \mathrm{~cm}$, decay stage 4) in a spruce-dominated mesic heath forest, 11 Sept. 2017, leg. TH 20170035 (OULU), det. MK.

Pseudotomentella nigra (Höhn. \& Litsch.) Svrček

Distribution. New to 2b, 3 b (Fig. 1). 


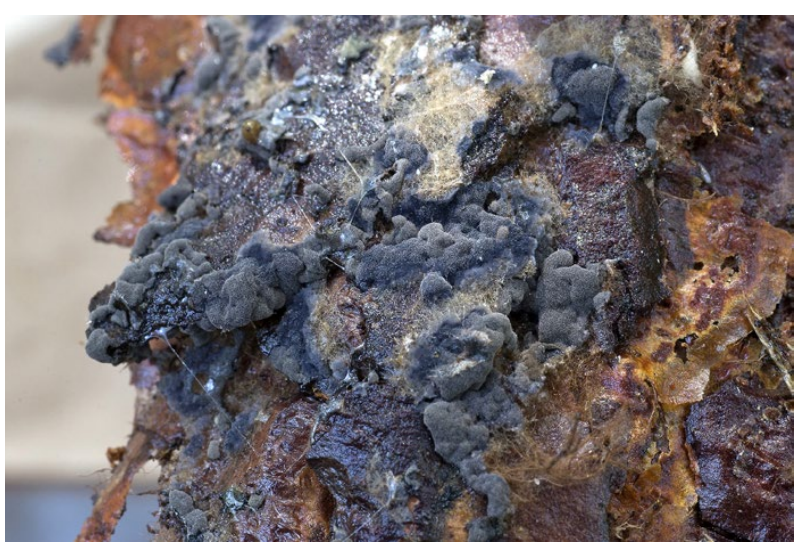

Figure 31. Pseudotomentella alobata in Kajaani (TH 20170035). Photo: Teppo Helo.

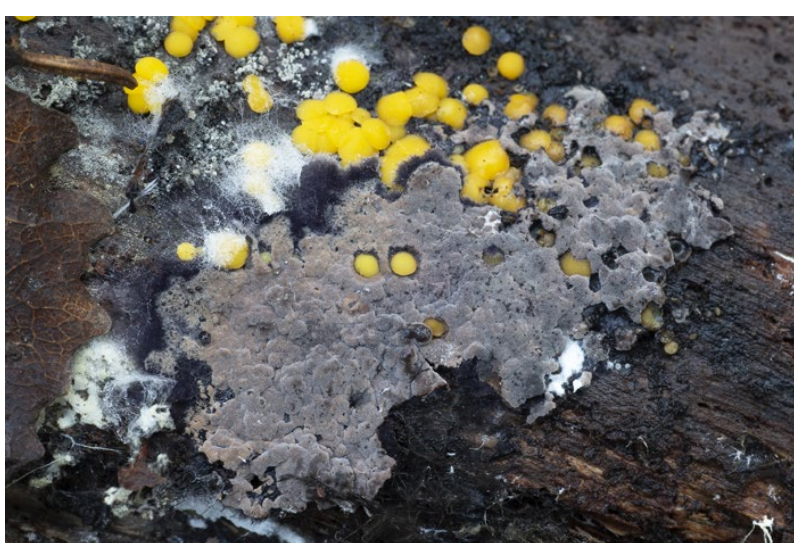

Figure 32. Pseudotomentella nigra in Kuhmo (TH 20200060). Photo: Teppo Helo.

Specimens examined. Savonia australis, Punkaharju, Kokonharju Nature Reserve UCS 6857539:3621745, on a fallen branch of Pinus sylvestris (diam. $7 \mathrm{~cm}$, decay stage 3 ) in a pine dominated mesic heath forest, 21 Sept. 2009, leg. \& det. JP 1019 (H); Ostrobottnia kajanensis, Kuhmo, Ulvinsalo, UCS 7103764:3665002, on a fallen trunk of Populus tremula (diam. $15 \mathrm{~cm}$, decay stage 3), in a very old spruce-dominated mesic heath forest, 2 Oct. 2020, leg. TH 20200060 (OULU), det. MK; Sotkamo, Saukkoperä, UCS 7086573:3584216, on charred fallen trunk of coniferous tree (diam. $45 \mathrm{~cm}$, decay stage 4 ), on the border between an old spruce-dominated mesic heath forest and a clear-cut area, 1 Sept. 2008, leg. P. Helo 1712 (OULU), det. TH \& MK; Sotkamo, Talvivaara UCS 7093224:3557672, on a fallen trunk of Picea abies (diam. $20 \mathrm{~cm}$, decay stage 3), in an old spruce-dominated mesic heath forest, 24 Sept. 2020, leg. TH 20200059 (OULU), det. MK.

\section{Pseudotomentella sciastra Svantesson \& Kõljalg}

Distribution. New to 3a (Fig. 1).

Notes. $3^{\text {rd }}$ record in Finland; previous records: Lammi (2a) and Jyväskylä (2b) (Svantesson et al. 2019). Note: The species is described from the Pseudotomentella tristis group. Most of the P. tristis specimens in the collections are determined incorrectly, so the distribution of the species is not reliably known. Probably quite rare.

Specimen examined. Ostrobottnia ouluensis, Oulu, Karjasilta, UCS 72128:34298, on a fallen trunk of Populus tremula, in an aspen-dominated herb-rich heath forest adjacent to a road, 18 Oct. 2019, leg. AM 229 (OULU), det. MK.

\section{Pseudotomentella umbrina (Fr.) M.J.Larsen (Fig. 33)}

Distribution. New to $3 \mathrm{a}-\mathrm{c}$ (Fig. 1).

Notes. $5^{\text {th }}-12^{\text {th }}$ records in Finland; previous records: Parainen (1b), Lammi (2a), Ikaalinen (2a), and Ruovesi (2b) (Svantesson et al. 2019). Note: The species was separated from the Pseudotomentella tristis group. The most common species in this group.

Specimens examined. Karelia australis, Virolahti, Haukijärvi, UCS 6715010:3527296, on a fallen Betula sp. trunk (diam. $30 \mathrm{~cm}$, decay stage 3) in a middle-aged - old Picea abies dominated herb-rich heath forest - mesic heath forest, 13 Sept. 2016, leg. JJ840 (OULU), det. MK; Ostrobottnia ouluensis, Oulu, Hietasaari, UCS 7213936:3426170, on a partly decorticated fallen trunk of Salix sp. (diam. $2 \mathrm{~cm}$, decay stage 2) in a lush deciduous-dominated coastal mixed forest, 12 Sept. 2011, leg. MK 40/11 \& P. \& M. Helo (OULU), det. MK; Ostrobottnia ouluensis, Oulu, Hietasaari, UCS 7213902:3426075, on a partly decorticated fallen trunk of Salix sp. (diam. $4 \mathrm{~cm}$, decay stage 2) in a dense and lush deciduous-dominated coastal mixed forest, 28 Sept. 2014, leg. \& det. MK 37/14 (OULU); Ostrobottnia ultima, Rovaniemi, Pisavaara Strict Nature Reserve, Sorvanulkki, UCS 7358625:3416764, on a partly decorticated fallen trunk of Picea abies (diam. $35 \mathrm{~cm}$, decay stage 4) in an old coniferous-dominated heath forest, 3 Oct. 2013, leg. \& det. MK 56/13 (OULU); Ostrobottnia ultima, Rovaniemi, Pisavaara Strict Nature Reserve, Pitkäjänkä, UCS 7359110:3415712, on a partly decorticated stump of Picea abies (diam. $12 \mathrm{~cm}$, decay stage 3) in an old coniferous-dominated mesic heath forest, 19 Aug. 2016, leg. MK 56/13 \& A. Ruotsalainen (OULU), det. MK; Ostrobottnia kajanensis, Sotkamo, Losonvaara UCS 7107123:3544783, on a fallen trunk of Sorbus aucuparia (diam. $5 \mathrm{~cm}$, decay stage 2), in an old spruce-dominated mesic heath forest, 15 Sept. 2020, leg. TH 20200063 (OULU), det. MK; Sotkamo, Talvivaara UCS 7092990:3557856, on a fallen trunk of Pinus sylvestris (diam. $12 \mathrm{~cm}$, decay stage 2), in an old spruce-dominated mesic heath forest, 22 Sept. 2020, leg. TH 20200064 (OULU), det. MK; Sotkamo, Korkeakoskenpuro UCS 7089332:3558290, on a fallen trunk of Populus tremula (diam. $10 \mathrm{~cm}$, decay stage 3 ), in an old spruce-dominated herb-rich heath forests, 24 Sept. 2018, leg. TH 20200223 (OULU), det. MK.

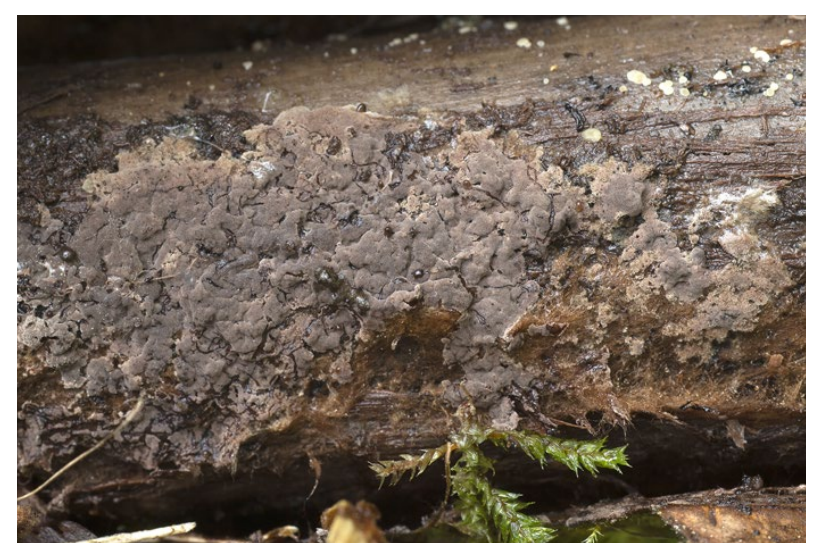

Figure 33. Pseudotomentella umbrina in Sotkamo (TH 20200063). Photo: Teppo Helo.

\section{Pseudoxenasma verrucisporum K.H. Larss.} \& Hjortstam

(Fig. 34)

Distribution. New to Finland, and hence new to $1 \mathrm{~b}$ (Fig. 1). 


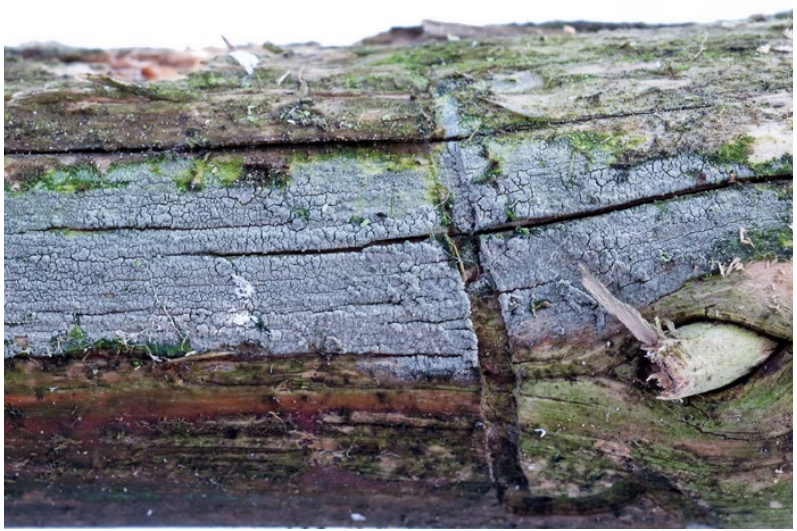

Figure 34. Pseudoxenasma verrucisporum in Helsinki (OM 24542). Photo: Otto Miettinen.

Specimen examined. Uusimaa, Helsinki, Haltiala, UCS 6684747:3385328, dead, attached branch (diam. $2 \mathrm{~cm}$, decay stage 2) of a recently died, standing Picea abies (diam. $60 \mathrm{~cm}$ ) with Globulicium hiemale in an old, dense spruce forest, $28 \mathrm{Feb}$. 2021 OM 24542 (H 6200144).

\section{Punctularia strigosozonata (Schw.) Talbot}

(Fig. 35)

Notes. $7^{\text {th }}-8^{\text {th }}$ records in Finland; previous records: Vehkalahti (2a), Savonranta (2b; two sites), Ilomantsi (2b and 3b; two sites), and Lieksa (3b) (Kotiranta et al. 2009). Vulnerable.

Specimens examined. Nylandia, Porvoo, Humla, UCS 6696:3430, on crown branches of a fallen dead Populus tremula, 19 Feb. 2020, leg. P. von Bagh, det. PV (H6083193), for site details see von Bagh (2020); Tavastia australis, Kouvola, Raajärvi UCS 6777967:3468376, on a fallen trunk of Populus tremula (diam. $35 \mathrm{~cm}$, decay stage 2) in a clearcut area, 13 May 2020, leg. \& det. JP4274 (H).

Repetobasidium vile (Bourd. \& Galz.) J. Erikss.

(Fig. 36)

Notes. $5^{\text {th }}$ record in Finland; previous records: Tammisaari (1b), Parainen (1b), Jyväskylä (2b), and Sotkamo (3b) (Kotiranta et al. 2009; Kunttu et al. 2014, 2019).

Specimen examined. Ostrobottnia kajanensis, Kuhmo, Ulvinsalo, UCS 7103332:3664690, on a fallen trunk of Picea abies (diam. $40 \mathrm{~cm}$, decay stage 3), in a very old spruce-dominated mesic heath forest, 4 Oct. 2020, leg. \& det. TH 20200065 (OULU).

\section{Saccosoma farinacea (Höhn.) Spirin \& K. Põldmaa}

[syn. Helicogloea farinacea (Höhn.) D. P. Rogers]

Distribution. New to $2 b$ (Fig. 1).

Notes. $8^{\text {th }}$ record in Finland; previous records: Karjalohja (1b), Helsinki (1b), Lempäälä (2a), Tampere (2a), Padasjoki (2a), Kajaani (3b), and Paltamo (3b) (Kotiranta et al. 2009; Kunttu et al. 2013; Kunttu et al. 2018; Kunttu et al. 2020).

Specimen examined. Savonia borealis, Lapinlahti, Koirniemi, 7033:3548, in crown branches of a fallen old Populus tremula retention tree, in a young herb-rich heath forest dominated by Picea abies and Betula pendula, 16 Jun. 2020, leg. \& det. PV $1935(\mathrm{H})$ as Saccosoma cf farinaceum, conf. V. Spirin.

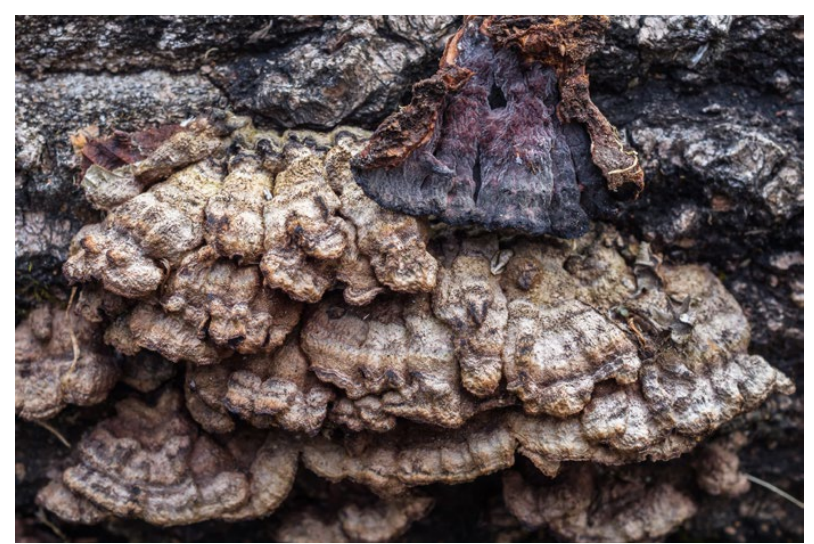

Figure 35. Punctularia strigosozonata in Kouvola (JP4274). Photo: Jorma Pennanen.

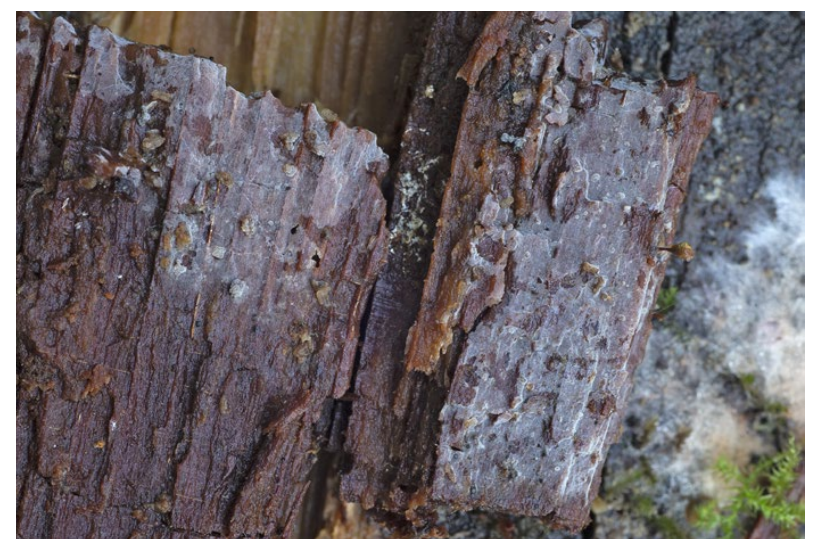

Figure 36. Repetobasidium vile in Kuhmo (TH 20200065). Photo: Teppo Helo.

\section{Scytinostroma praestans (H.S. Jacks.) Donk}

Distribution. New to $4 d$ (Fig. 1).

Specimen examined. Lapponica enontekiensis, Enontekiö, Enontekiö, Jogasjávri, Doskaljohka W, UCS 768596:328070, on a dead trunk or branch of Betula nana, in a moist to dry mountain birch forest, 27 Jul. 2020 H. Väre 25322 (H) det. HK.

Sidera vulgaris (Fr.) Miettinen

(Fig. 37)

Notes. $9^{\text {th }}$ record in Finland; previous records: Nauvo (1b), Kemiönsaari (1b) Kirkkonummi (1b), Helsinki (1b \& 2a; four sites), and Sipoo (2a) (Vauras 2000; Kunttu

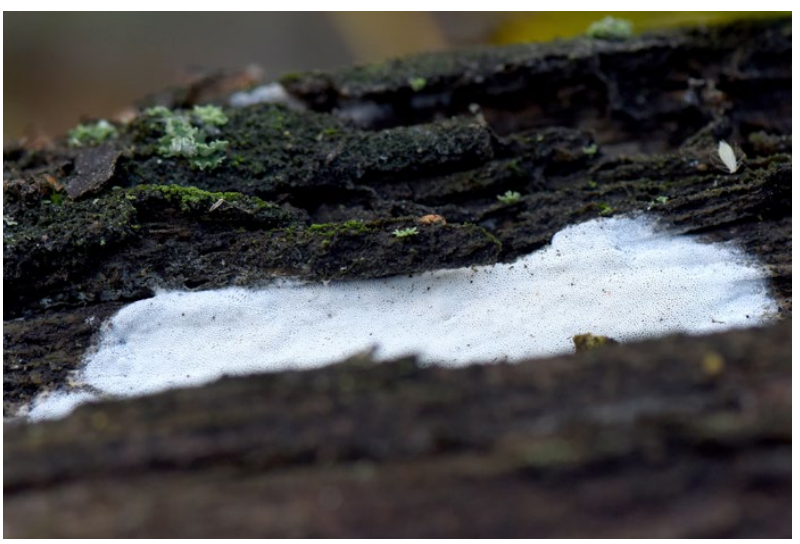

Figure 37. Sidera vulgaris in Helsinki (PV 2572). Photo: Pyry Veteli. 
et al. 2014, 2016; Savola 2015; Savola \& Kolehmainen 2015). Near Threatened.

Specimen examined. Nylandia, Helsinki, Kustaankartano, UCS 6681:3386, on Sorbus aucuparia (diam. 12 cm, decay stage 3), herb-rich site with mixed stand of old conifers and Salix caprea, Betula pendula, Sorbus aucuparia, Populus tremula, Alnus incana, Ulmus glabra and Acer platanoides etc, with high amounts of coarse woody debris, 17 Oct. 2020, leg. \& det. PV $2572(\mathrm{H})$.

\section{Sistotrema oblongisporum M.P. Christ. \& Hauersl.}

Distribution. New to 3a (Fig. 1).

Specimen examined. Ostrobottnia ouluensis, Oulu, Kontinkangas, UCS 7212832:3429839, on a fallen trunk of Populus trem$u l a$, in an aspen-dominated herb-rich heath forest adjacent to a road, 12 Oct. 2019, leg. \& det. AM 143 (OULU), conf. MK.

\section{Sistotrema subtrigonospermum D. P. Rogers (Fig. 38)}

Distribution. New to Finland, and hence new to $3 \mathrm{~b}$ (Fig. 1).

Specimen examined. Ostrobottnia kajanensis, Sotkamo, Losonvaara, UCS 7107251:3544808, on a fallen branch of deciduous tree (diam. $1.5 \mathrm{~cm}$, decay stage 3 ) in an old spruce-dominated mesic heath forest, 15 Sept. 2020, leg. \& det. TH 20200127 (OULU), conf. MK; at the same site UCS 7106777:3545232, on a fallen branch of Picea abies (diam. $1 \mathrm{~cm}$, decay stage 4) with Botryobasidium laeve in an old spruce-dominated mesic heath forest, 30 Sept. 2020, leg. \& det. TH 20200128 (OULU), conf. MK.

\section{Spiculogloea minuta P. Roberts}

Distribution. New to Finland, and hence new to $3 \mathrm{~b}$ (Fig. 1).

Specimen examined. Ostrobottnia kajanensis, Sotkamo, Talvivaara, UCS 7093211:3557817, on a fallen trunk of Picea abies (diam. $15 \mathrm{~cm}$, decay stage 3) with Tulasnella allantospora in an old spruce-dominated mesic heath forest, 23 Sept. 2020, leg. TH 20200008 (H), det. MK \& TH.

\section{Spiculogloea subminuta Hauerslev}

Distribution. New to 2b, 3a, c (Fig. 1).

Notes. $4^{\text {th }}-10^{\text {th }}$ records in Finland; previous records: Kuhmo (3b), Kajaani (3b), and Inari (4c) (Kunttu et al. 2019, 2020).

Specimen examined. Karelia borealis, Värtsilä, Patsola, Savikko, Rauhanmaja W, UCS 69077:36888, on a fallen trunk of Picea abies in a spruce-dominated brookside forest, 24 Jun. 1995, leg. H. Väre (OULU), det. MK; Ostrobottnia media, Pyhäjärvi, Mäkikylä, Iso Kärsämäenjärvi, Kokkopuro, UCS 70904:34616, on a decorticated fallen trunk of Pinus sylvestris (diam. $15 \mathrm{~cm}$ ) with Botryobasidium subcoronatum (Höhn. \& Litsch.) Donk, in a moist coniferous-dominated brookside mixed forest, 27 Oct. 1998, leg. \& det. MK 116/98 (OULU); Ostrobottnia media, Pyhäjärvi, Mäkikylä, Kärsämäenjärvet, Isolehto, UCS 70918:34632, on a fallen trunk of Picea abies (diam. $19 \mathrm{~cm}$ ) with Botryobasidium subcoronatum (Höhn. \& Litsch.) Donk, in an old spruce-dominated mesic heath forest, 26 Sept. 1999, leg. \& det. MK 136/99 (OULU); Ostrobottnia ouluensis, Pudasjärvi, Sarvisuo, UCS 7223013:3493616, on a fallen trunk of Alnus incana with Botryobasidium subcoronatum (Höhn.

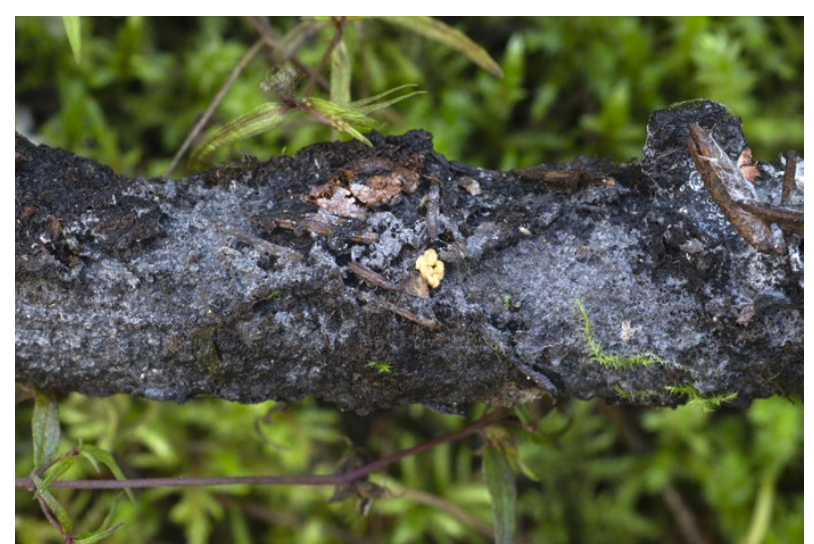

Figure 38. Sistotrema subtrigonospermum in Sotkamo (TH 20200127). Photo: Teppo Helo.

\& Litsch.) Donk, in a spruce-dominated herb-rich forest next to a stream running from a spring, 10 July 2018 , leg. AM 46-18 (OULU), det. MK; Ostrobottnia kajanensis, Puolanka, Paljakka Strict Nature Reserve, Kaijansuo W, UCS 7183458:3550223, on a fallen trunk of Picea abies (diam. $50 \mathrm{~cm}$, decay stage 3) with Botryobasidium subcoronatum (Höhn. \& Litsch.) Donk, in an old spruce-dominated mesic heath forest, 26 Sept. 2003, leg. MK 56/03b \& P. Halonen (OULU), det. MK; Ostrobottnia ultima, Rovaniemi, Pisavaara Strict Nature Reserve, UCS 7359420:3416576, on a decorticated fallen trunk of Picea abies (diam. $20 \mathrm{~cm}$, decay stage 4) with Botryobasidium subcoronatum (Höhn. \& Litsch.) Donk, in an old mesic heath forest, 17 Sept. 2015. leg. MK 66/15 \& P. Helo (OULU), det. MK; Ostrobottnia ultima, Rovaniemi, Pisavaara Strict Nature Reserve, Alalaki E, UCS 7350666:3413522, on a decorticated partly charred fallen trunk of Pinus sylvestris (diam. $30 \mathrm{~cm}$, decay stage 1) with Botryobasidium subcoronatum (Höhn. \& Litsch.) Donk, in an old mesic heath forest, 11 Sept. 2020, leg. \& det. MK 27/20 (OULU).

Tomentella botryoides (Schwein.) Bourd. \& Galz.

(Fig. 39)

Distribution. New to Finland and hence new to $1 \mathrm{~b}$ (Fig. 1).

Specimen examined. Regio aboënsis, Turku, Ruissalo, Choraeus Bridge, UCS approx. 6712:3234, on a fallen trunk of Quercus robur, 9 Sept. 1937, leg. Matti Laurila, (TUR), det. MK; UCS 6711:3233, on a fallen branch of Quercus robur (diam. 10, decay stage 2) in a small herb-rich forest with oak trees, 30 Sept. 2019, leg. \& det. TH 2019023 (OULU), conf. MK \& E. Martini.

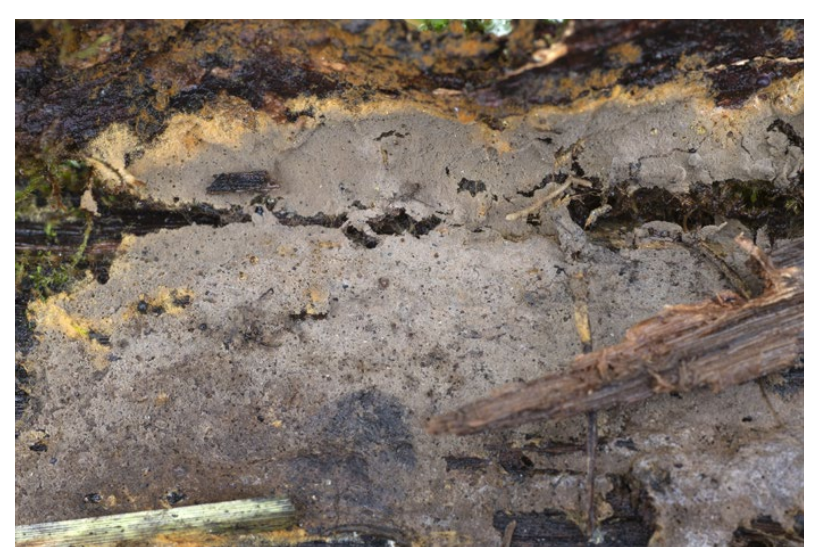

Figure 39. Tomentella botryoides in Turku (TH 2019023). Photo: Teppo Helo. 
Tomentella ellisii (Sacc.) Jülich \& Stalpers

(Fig. 40)

Distribution. New to 3 b (Fig. 1).

Note. This is probably a group of species. Therefore, the distribution of the species is not reliable.

Specimen examined. Ostrobottnia kajanensis, Sotkamo, Penikkapuro UCS 7087271:3559145, on a fallen trunk of Populus tremula (diam. $7 \mathrm{~cm}$, decay stage 3 ) in an old spruce-dominated mesic heath forest around a stream, 25 Sept. 2018, leg. \& det. TH 20180220 (OULU), conf. MK \& E. Martini.

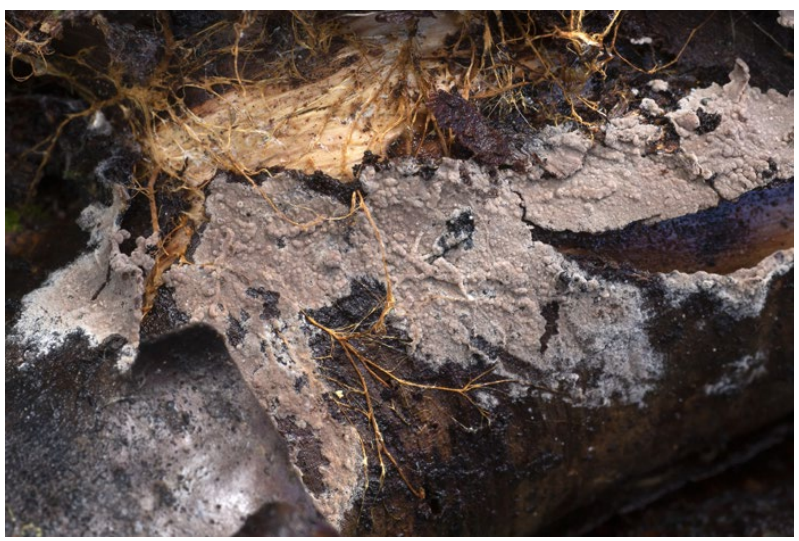

Figure 40. Tomentella ellisii in Sotkamo (TH 20180220). Photo: Teppo Helo.

Tomentella fuscocinerea (Pers.: Fr.) Donk

(Fig. 41)

Distribution. New to 3 b (Fig. 1).

Notes. $2^{\text {nd }}$ record in Finland; previous record: Kemiönsaari (1b) (Kunttu et al. 2015).

Specimen examined. Ostrobottnia kajanensis, Kuhmo, Ulvinsalo, UCS 7103751:3665047, on a fallen trunk of Populus tremula (diam. $5 \mathrm{~cm}$, decay stage 2), in a very old spruce-dominated mesic heath forest, 2 Oct. 2020, leg. \& det. TH 20200004 (OULU, H), conf. MK.

\section{Tomentella galzinii Bourdot}

(Fig. 42)

Distribution. New to 3a-b (Fig. 1).

Specimens examined. Ostrobottnia ouluensis, Oulu, Kontinkangas, UCS 7212832:3429839, on a fallen trunk of Populus tremula, in an aspen-dominated herb-rich heath forest next to a road, 12 Oct. 2019, leg. \& det. AM 130 (OULU), conf. E. Martini; Ostrobottnia kajanensis, Paltamo, Melalahti UCS 71471:35327, on a fallen trunk of Salix caprea (diam. 2 cm, decay stage 2) in a calcareous mesic eutrophic herb-rich forest, 24 Sept. 2019, leg. \& det. TH 20190052 (OULU), conf. MK

\section{Tomentella lapida (Pers.) Stalpers}

(Fig. 43)

Distribution. New to 2b, $3 b$ (Fig. 1).

Notes. $2^{\text {nd }}-8^{\text {th }}$ records in Finland; previous record: Noormarkku (2a) (Kotiranta et al. 2009). There are more than 40 collections from the Kainuu region recorded between 2018-2020 (T. Helo, unpublished). Note: Tomentella specimens are often incorrectly identified. There are probably more of this species in Finnish collections.

Specimens examined. Tavastia borealis, Toivakka, Vuorilampi Nature Reserve, UCS 68852:34558, on Populus tremula, leg.

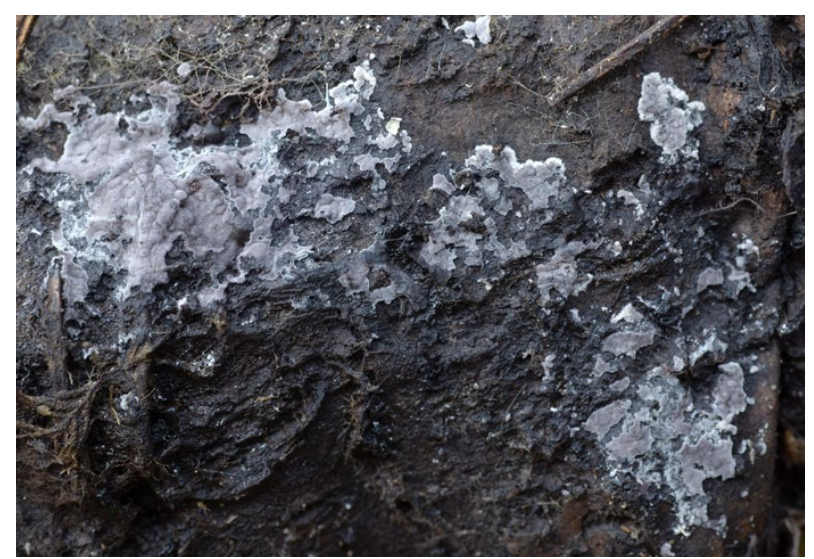

Figure 41. Tomentella fuscocinerea in Kuhmo (TH 20200004). Photo: Teppo Helo.

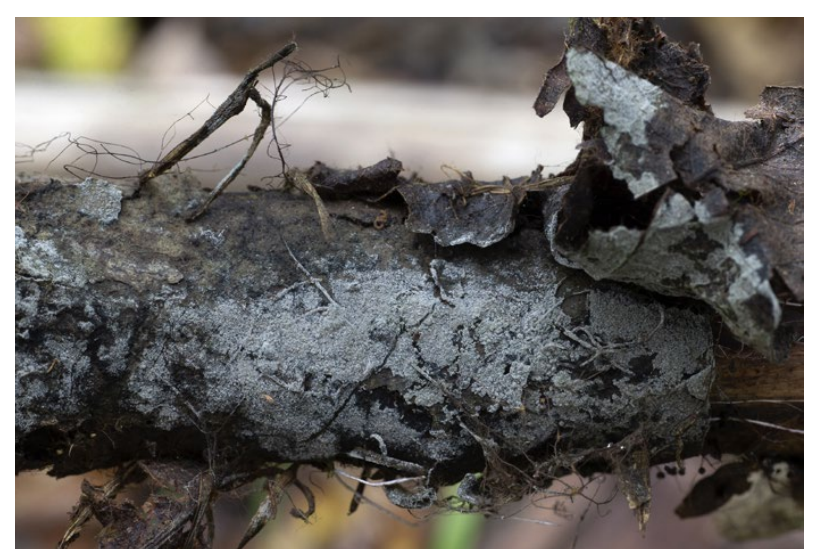

Figure 42. Tomentella galzinii in Paltamo (TH 20190052). Photo: Teppo Helo.

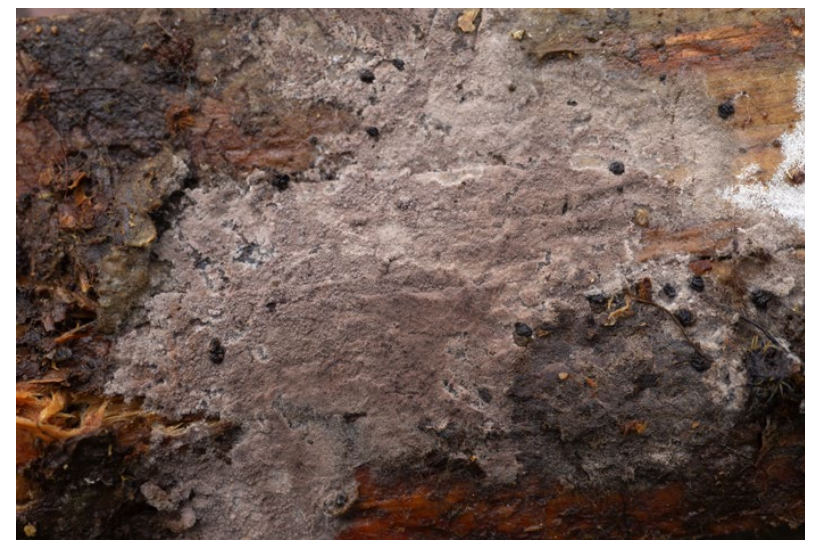

Figure 43. Tomentella lapida in Sotkamo (TH 20200066). Photo: Teppo Helo.

\& det. J. Purhonen (JYV); Ostrobottnia kajanensis, Hyrynsalmi, Karhisenvaara, UCS 7157343:3587655, on a fallen trunk of Populus tremula (diam. $35 \mathrm{~cm}$, decay stage 2 ) in an old spruce-dominated mesic heath forest, 28 Sept. 2018, leg. \& det. TH 20180221 (OULU), conf. MK; Paltamo, Melalahti, UCS 71471:35327, on a fallen trunk of Salix caprea (diam. $3 \mathrm{~cm}$, decay stage 4) in a calcareous mesic eutrophic herb-rich forest, 24 Sept. 2019, leg. \& det. TH 20190053 (OULU), conf. MK; Sotkamo, Losonvaara, UCS 3545317:7106808, on a fallen trunk of Picea abies (diam. $28 \mathrm{~cm}$, decay stage 3 ) in an old spruce-dominated mesic heath forest, 14 Aug. 2020, leg. \& det. TH 20200066 (OULU, H), conf. MK \& E. Martini; Puolanka, Taapuri, UCS 7106789:3545178, on a fallen trunk of Populus tremula (diam. $35 \mathrm{~cm}$, decay stage 3 ) in 
an old spruce-dominated mesic heath forest, 17 Aug. 2020, leg. \& det. TH 20200067 (OULU), conf. MK; Sotkamo, Talvivaara, UCS 7092390:3558388, on a fallen trunk of Populus tremula (diam. $8 \mathrm{~cm}$, decay stage 3 ) in an old spruce-dominated mesic heath forest, 21 Aug. 2020, leg. \& det. TH 20200122 (OULU), conf. MK; Kuhmo, Elimyssalo UCS 71265:36593, on a fallen trunk of Picea abies (diam. $6 \mathrm{~cm}$, decay stage 3) with Piloderma byssinum in an old spruce-dominated mesic heath forest, 6 Oct. 2020, leg. TH 20200068 (OULU), det. MK.

\section{Tomentella lateritia Pat.}

(Fig. 44)

Distribution. New to 3 b (Fig. 1).

Specimen examined. Ostrobottnia kajanensis, Sotkamo, Losonvaara, UCS 7105320:3545502, on a fallen trunk of Picea abies (diam. $14 \mathrm{~cm}$, decay stage 3 ) in an old spruce-dominated mesic heath forest, 31 Aug. 2005, leg. P. Helo 1455 (OULU), det. E. Martini; Sotkamo, Losonvaara, UCS 7107300:3545992, on a fallen trunk of Juniperus communis (diam. $4 \mathrm{~cm}$, decay stage 4) with Piloderma sphaerosporum in a middle-aged spruce-dominated mesic heath forest, 28 Sept. 2020, leg. \& det. TH 20200005 (OULU, H), conf. MK.

\section{Tomentella neobourdotii M.J. Larsen}

(Fig. 45)

Distribution. New to Finland and hence new to $3 \mathrm{a}-\mathrm{b}$ (Fig. 1).

Specimens examined. Ostrobottnia ouluensis, Oulu, Nuottasaari, UCS 7212460:3426775, on a corticated fallen trunk of Betula sp. (diam. $13 \mathrm{~cm}$, decay stage 4) in a lush deciduous-dominated mixed forest, 10 Sept. 2014 leg. MK 20/14 (OULU), det. E. Martini; Ostrobottnia kajanensis, Paltamo, Melalahti UCS 71471:35327, on a fallen trunk of Betula sp. (diam. $18 \mathrm{~cm}$, decay stage 4) in a calcareous mesic eutrophic herb-rich forest, 24 Sept. 2019, leg. \& det. TH 20190054 (OULU), conf. MK; Paltamo, Antinmäki UCS 71389:35486, on a fallen trunk of Salix caprea (diam. $15 \mathrm{~cm}$, decay stage 2) in a mesic mesotrophic herbrich forest, 20 Aug. 2019, leg. \& det. TH 20190055 (OULU), conf. MK; Ristijärvi, Saukkovaara UCS 7151866:3559621, on a fallen trunk of Populus tremula (diam. $4 \mathrm{~cm}$, decay stage 2) in a middle-aged deciduous-dominated herb-rich heath forest, 12 Oct. 2018, leg. \& det. TH 20180222 (OULU), conf. MK

\section{Tomentella subpilosa Litsch.}

(Fig. 46)

Distribution. New to Finland, and hence new to $2 \mathrm{a}, 3 \mathrm{~b}$ (Fig. 1).

Specimen examined. Satakunta, Pori, Metallinkylä, UCS 6827887:3225781, on a fallen trunk of Salix caprea (diam. $10 \mathrm{~cm}$, decay stage 3) in a forested old field, 2 Oct. 2019, leg. H. Lehtonen 2.10.19/16 (OULU), det. TH \& MK, conf. E. Martini. Ostrobottnia kajanensis, Sotkamo, Losonvaara, UCS 7106805:3545188, on a fallen trunk of Populus tremula (diam. $35 \mathrm{~cm}$, decay stage 4) with Trechispora kavinioides in an old spruce-dominated mesic heath forest, 14 Aug. 2020, leg. TH 20200069 (OULU, H), det. TH \& MK, conf. E. Martini.

\section{Tomentella subtestacea Bourdot \& Galzin}

(Fig. 47)

Distribution. New to Finland and hence new to $3 \mathrm{a}-\mathrm{b}$ (Fig. 1).

Specimens examined. Ostrobottnia ouluensis, Oulu, Hietasaari, UCS 7213925:3426179, on a partly decorticated piece of Alnus incana on the ground (diam. $12 \mathrm{~cm}$, decay stage 3 ) in a dense and lush deciduous-dominated coastal mixed forest, 28 Sept. 2014 ,

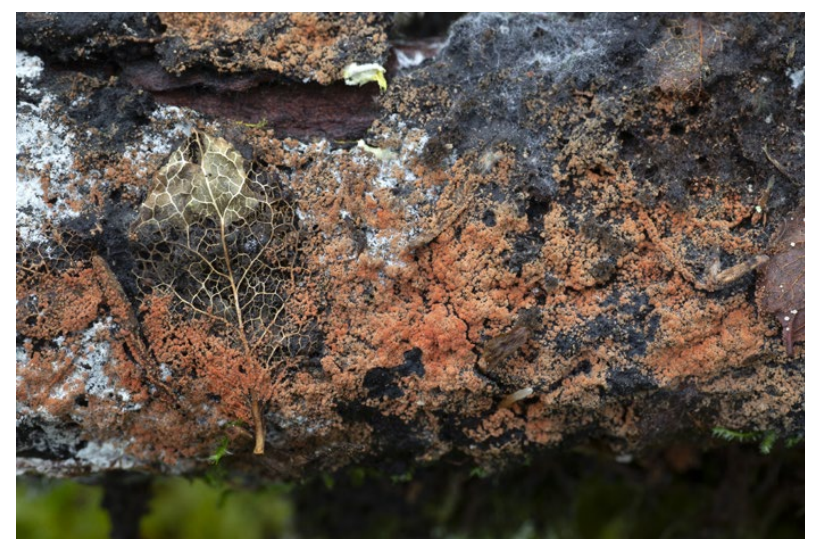

Figure 44. Tomentella lateritia in Sotkamo (TH 20200005) with Piloderma sphaerosporum. Photo: Teppo Helo.

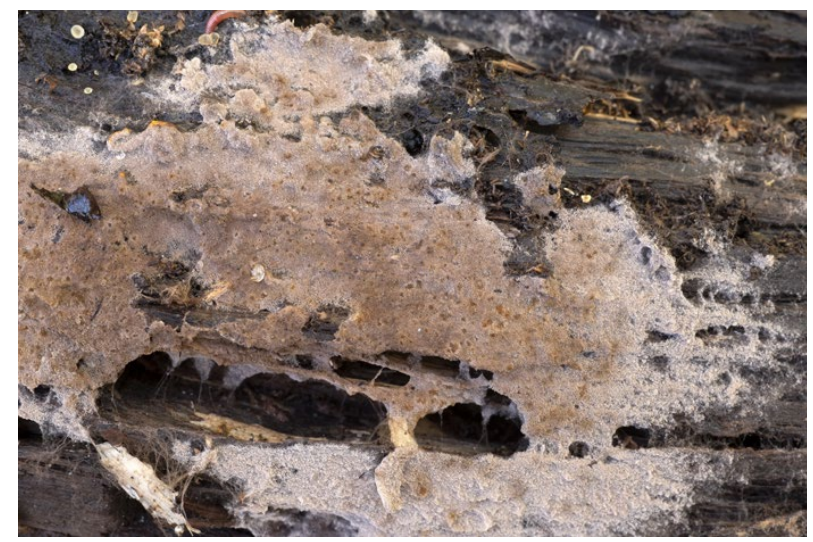

Figure 45. Tomentella neobourdotii in Paltamo (TH 20190054). Photo: Teppo Helo.

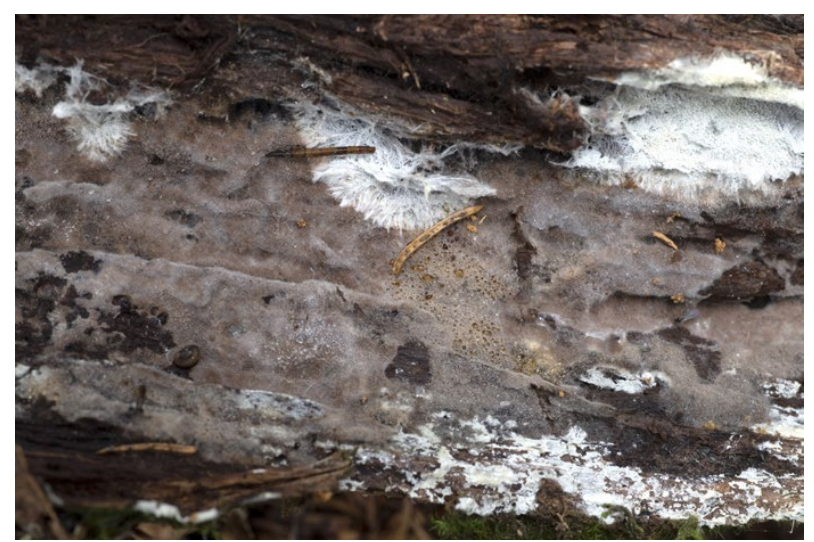

Figure 46. Tomentella subpilosa in Sotkamo (TH 20200069). Photo: Teppo Helo.

leg. MK 45/14 (OULU), det. E. Martini; Ostrobottnia kajanensis, Paltamo, Tololanmäki, UCS 7137:3551, on a fallen trunk of deciduous tree (diam. $6 \mathrm{~cm}$, decay stage 3 ) in a spruce-dominated mesic mesotrophic herb-rich forest, 18 Aug. 2017, leg. TH 20170036 (OULU), det. MK.

Tomentella terrestris (Berk. \& Broome) M.J. Larsen (Fig. 48)

Distribution. New to 3c (Fig. 1).

Notes. $4^{\text {th }}-12^{\text {th }}$ records in Finland; previous records: Kemiönsaari (1b), Vehkalahti (2b), and Kuhmo (3b) (Kotiranta et al. 2009; Kunttu et al. 2012). Altogether there are 


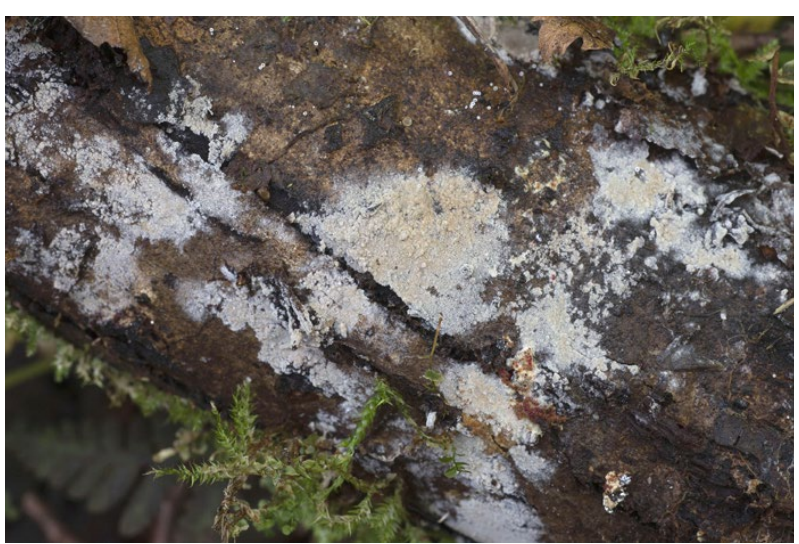

Figure 47. Tomentella subtestacea in Paltamo (TH 20170036). Photo: Teppo Helo.

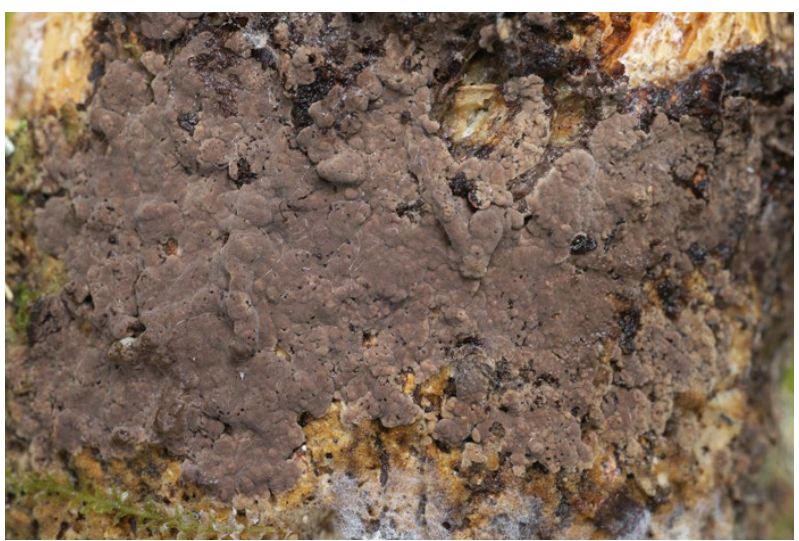

Figure 48. Tomentella terrestris in Sotkamo (TH 20200126). Photo: Teppo Helo.

approximately 20 collections from the Kainuu province, only a selection is presented here. Note: Tomentella specimens are often incorrectly identified. There are probably more of this species in Finnish collections.

Specimens examined. Tavastia borealis, Rautalampi, Iso Niinivuori, UCS 6934420:3487177, on a decorticated fallen trunk of Juniperus communis (diam. $6 \mathrm{~cm}$, decay stage 1) in a mesic heath forest, 16 Sept. 2017, leg. MK 39/17 \& et al., OULU, det. MK; Ostrobottnia kajanensis, Sotkamo, Losonvaara, UCS 7106795:3545403, on a fallen trunk of deciduous tree (diam. $4 \mathrm{~cm}$, decay stage 2) in an old spruce-dominated mesic heath forest, 14 Aug. 2020, leg. \& det. TH 20200125 (OULU); and at the same site UCS 7107180:3544769, on a fallen trunk of Picea abies (diam. $17 \mathrm{~cm}$, decay stage 3) in an old spruce-dominated mesic heath forest, 15 Sept. 2020, leg. \& det. TH 20200126 (OULU); Puolanka, Taapuri, UCS 7164697:3541850, on a fallen trunk of Betula sp. (diam. $20 \mathrm{~cm}$, decay stage 3 ) in an old spruce-dominated mesic heath forest, 17 Aug. 2020, leg. \& det. TH 20200123 (OULU); Sotkamo, Talvivaara, UCS 7092452:3558387, on a fallen trunk of Picea abies (diam. $20 \mathrm{~cm}$, decay stage 3 ) in an old spruce-dominated mesic heath forest, 21 Aug. 2020, leg. \& det. TH 20200124 (OULU); Sotkamo, Viltonvaara UCS 7084168:3561684, on a fallen trunk of Betula sp. (diam. $18 \mathrm{~cm}$, decay stage 3) in an old spruce-dominated mesic heath forest, 1 Oct. 2018, leg. \& det. TH 20200223 (OULU); Hyrynsalmi, Karhisenvaara UCS 7157342:3587734, on a fallen trunk of Populus tremula (diam. $25 \mathrm{~cm}$, decay stage 3) in an old spruce-dominated mesic heath forest, 28 Sept. 2018, leg. \& det. TH 20200224 (OULU): Ostrobottnia ultima, Tervola, Pisavaara Strict Nature
Reserve, Alalaki, UCS 7350633:3413306, on a decorticated partly charred stump of Pinus sylvestris (decay stage 2) in an old dry heath forest, 2 Sept. 2020, leg. \& det. MK 20/20 (OULU) and on the same site UCS 7350612:3413510, on a decorticated fallen trunk of Pinus sylvestris (diam. $10 \mathrm{~cm}$, decay stage 3) in an old mesic heath forest, 11 Sept. 2020, leg. \& det. MK 26/20 (OULU).

\section{Tomentellopsis echinospora (Ellis) Hjortstam}

Distribution. New to $4 \mathrm{~b}$ (Fig. 1).

Specimen examined. Ostrobottnia ultima, Rovaniemi, Vanttauskoski, UCS 7371:3488, fallen trunk of Pinus sylvestris (diam. $26 \mathrm{~cm}$, decay stage 2), 24 Sept. 2020, leg. \& det. PV 2483 (H).

\section{Tomentellopsis submollis (Svrček) Hjortstam}

Distribution. New to 4 b (Fig. 1).

Specimen examined. Ostrobottnia ultima, Rovaniemi, Rättiselkä, UCS 7402:3464, fallen trunk of Pinus sylvestris (diam. $50 \mathrm{~cm}$, decay stage 5) in an Empetrum-Myrtillus heath forest with a high amount of dead wood, 17 Sept. 2020, leg. \& det. PV $2397(\mathrm{H})$.

\section{Tomentellopsis zygodesmoides Ellis \& Hjortstam}

Distribution. New to $3 b$ (Fig. 1).

Notes. $3^{\text {rd }}$ record in Finland; previous records: Kemiönsaari (1b) and Tuusula (2a) (Kotiranta et al. 2009; Kunttu et al. 2012).

Specimen examined. Ostrobottnia kajanensis, Sotkamo, Losonvaara UCS 7106771:3545279, on a fallen trunk of Populus tremula (diam. $8 \mathrm{~cm}$, decay stage 3 ) in an old spruce-dominated mesic heath forest, 30 Sept. 2020, leg. TH 20200070 (OULU), det. MK.

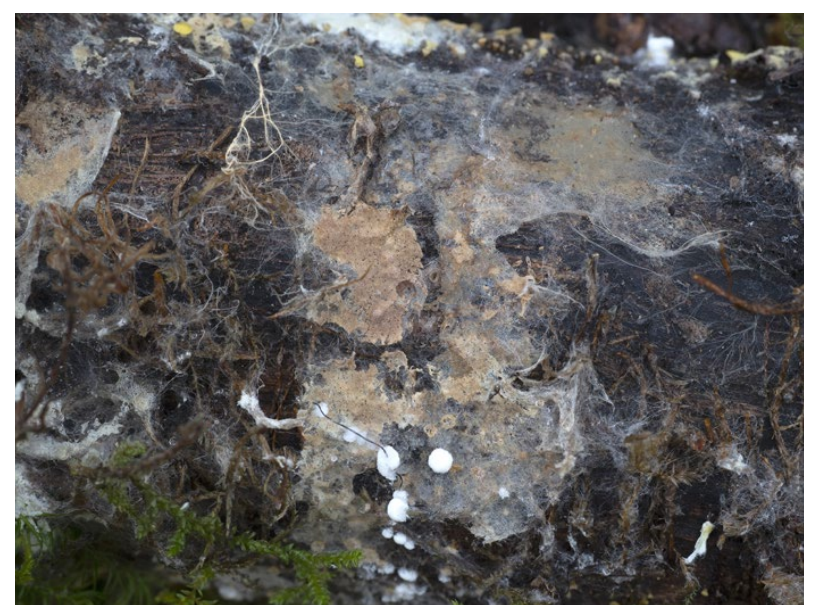

Figure 49. Tomentellopsis zygodesmoides in Sotkamo (TH 20200070). Photo: Teppo Helo.

\section{Trechispora incisa K.H. Larsson}

(Fig. 50)

Notes. $2^{\text {nd }}$ record in Finland; previous record: Lohja (1a) (Kotiranta et al. 2009).

Specimen examined. Nylandia, Raasepori, Karjaa, Lepinjärvi, UCS 6664277:3313401, on a broken trunk of Betula pendula (diam. $30 \mathrm{~cm}$, decay stage 4) in a mixed herb-rich heath forest, 28 Oct. 2020, leg. \& det. JP 4355 (H), conf. HK. 


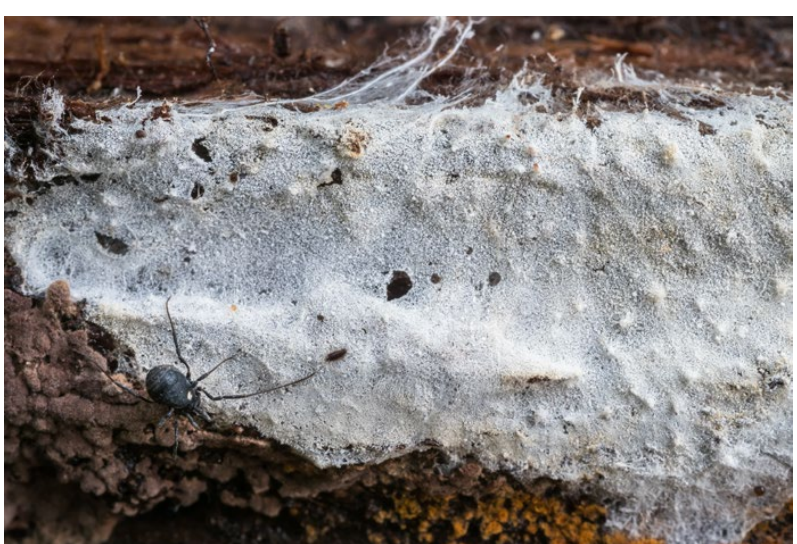

Figure 50. Trechispora incisa in Raasepori (JP 4355). Photo: Jorma Pennanen.

\section{Trechispora laevis K.H. Larsson}

Distribution. New to 3a (Fig. 1).

Specimen examined. Ostrobottnia ouluensis, Oulu, Knuutilankangas, UCS 72111:34313, on a stump of Populus tremula, in a moist brookside forest, 12 Oct. 2019, leg. AM 164 (OULU), det. MK; Ostrobottnia ouluensis, Oulu, Iinatti, UCS 7209158:3434103, on a branch of Pinus sylvestris (decay stage 3), in a small pine-dominated mesic heath forest, 6 May 2020, leg AM 284 (OULU), det. MK.

\section{Trechispora microspora (P. Karst.) Liberta}

(Fig. 51)

Distribution. New to 3a-b (Fig. 1).

Specimens examined. Ostrobottnia ouluensis, Utajärvi, Hanganvaara, Hanganhete, UCS 7220912:3490586, on a fallen trunk of Picea abies, in a spruce-dominated herb-rich forest next to a stream running from a spring, $14 \mathrm{Jul} .2018$, leg. \& det. AM 79-18 (OULU), conf. MK; Ostrobottnia kajanensis, Sotkamo, Losonvaara UCS 7107109:3544787, on a fallen trunk of Juniperus communis (diam. $1.5 \mathrm{~cm}$, decay stage 2) in an old spruce-dominated mesic heath forest, 15 Sept. 2020, leg. \& det. TH 20200071 (OULU), conf. MK.

\section{Trechispora minuta K.H. Larss.}

Distribution. New to 3a (Fig. 1).

Specimen examined. Ostrobottnia ouluensis, Oulu, Karjasilta, UCS 7212919:3429700, on a fallen trunk of Larix sibirica, in a small mesic heath forest with larch-trees planted next to the road, 26 Oct. 2019, leg. AM 274 (OULU), det. MK.

\section{Trechispora stellulata (Bourdot \& Galzin) Liberta}

Distribution. New to 4d (Fig. 1).

Specimen examined. Lapponica enontekiensis, Enontekiö, Jogasjávri N, Doskaljohka W, UCS 768596:328070, on a dead trunk or branch of Betula pubescens subsp. czerepanovii, in a moist to dry mountain birch forest, 27 Jul. 2020, H. Väre $25319(\mathrm{H})$, det. MK, conf. HK.

\section{Tremella polyporina D. A. Reid.}

Notes. $6^{\text {th }}$ record in Finland; previous records: Helsinki (1b), Lammi (2a), Tampere (2a), Hyrynsalmi (3b), and Inari (4c) (Pippola \& Kotiranta 2008; Kotiranta et al. 2009; Miettinen 2012; Kunttu et al. 2016).

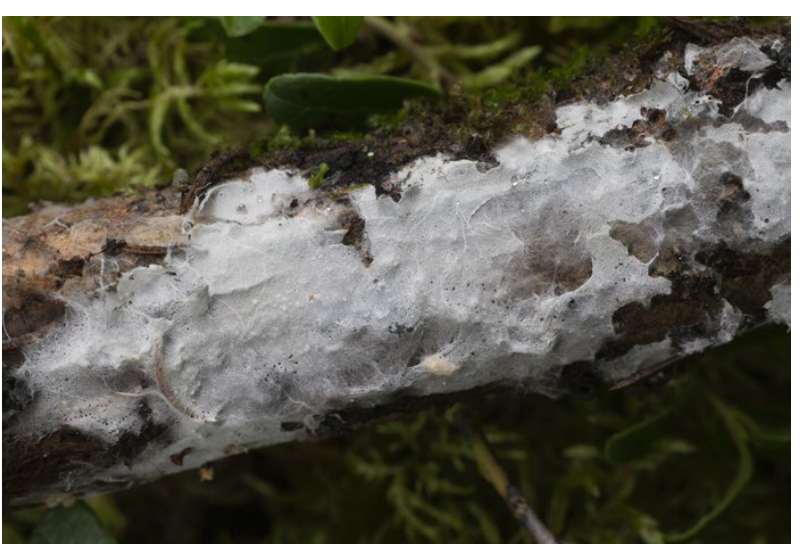

Figure 51. Trechispora microspora in Sotkamo (TH 20200071). Photo: Teppo Helo.

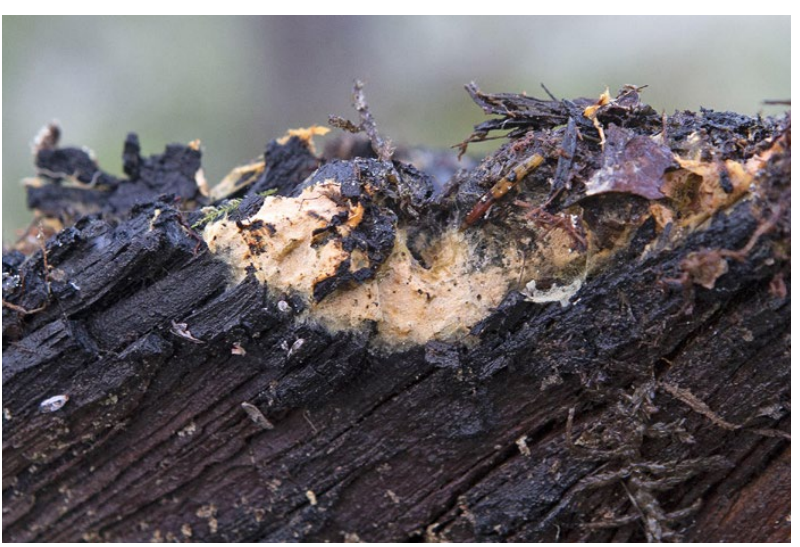

Figure 52. Tretomyces microsporus in Sotkamo (TH 20200072). Photo: Teppo Helo.

Specimen examined. Nylandia, Helsinki, Pirttipolunpuisto, UCS 6680:3385, fallen trunk of Picea abies (diam. $25 \mathrm{~cm}$, decay stage 3), inside a Postia sp. basidioma, 8 Nov. 2020, leg. \& det. PV $2616(\mathrm{H})$

Tretomyces microsporus Kotir., Saaren. \& K.H. Larss.

(Fig. 52)

Notes. $7^{\text {th }}$ record in Finland; previous records: Paltamo (3a), Oulu (3a), Lieksa (3b; two sites), Suomussalmi (4a), and Inari (4c) (Kotiranta et al. 2009; Kunttu et al. 2015, 2018, 2019).

Specimen examined. Ostrobottnia kajanensis, Sotkamo, Katajavaara UCS 71056:35956, on a charred stump of Pinus sylvestris (diam. $35 \mathrm{~cm}$, decay stage 4) in an old pine-dominated sub-xeric heath forest, 6 Nov. 2020, leg. \& det. TH 20200072 (OULU).

Tubulicrinis angustus (D.P. Rogers \& Weresub) Donk

(Fig. 53)

Distribution. New to 3b (Fig. 1).

Specimens examined. Ostrobottnia kajanensis, Sotkamo, Losonvaara UCS 710718:354477, on a fallen branch of Picea abies (diam. $1.5 \mathrm{~cm}$, decay stage 2) in an old spruce-dominated mesic heath forest, 15 Sept. 2020, leg. \& det. TH 20200073 (OULU), conf. MK; at the same site UCS 710718:354477, on a fallen branch of Populus tremula (diam. $2 \mathrm{~cm}$, decay stage 3 ) in an old spruce-dominated mesic heath forest, 15 Sept. 2020, leg. \& det. TH 20200074 (OULU), conf. MK. 


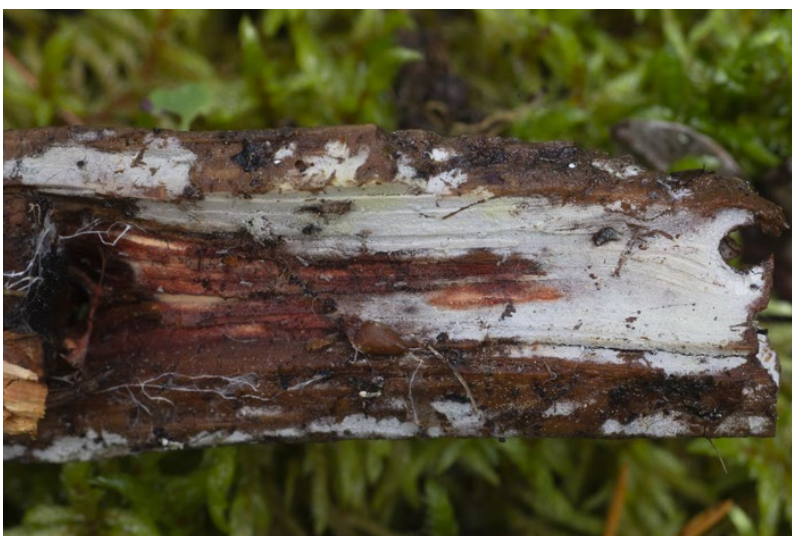

Figure 53. Tubulicrinis angustus in Sotkamo (TH 20200074). Photo: Teppo Helo.

Tubulicrinis effugiens (Bourdot \& Galzin)

(Fig. 54)

Distribution. New to 3c (Fig. 1).

Notes. $7^{\text {th }}-8^{\text {th }}$ records in Finland; previous records: Pihtipudas (3a), Lieksa (3b), Kuusamo (4a), Muonio (4b), Salla (4c), Savukoski (4c) (Kotiranta et al. 2009; H. Kotiranta, unpublished).

Specimens examined. Ostrobottnia kajanensis, Kuhmo, Ulvinsalo, UCS 7102550:3662494, on a fallen trunk of Pinus sylvestris (diam. $5 \mathrm{~cm}$, decay stage 3, kelo tree) in a middle-aged pine-dominated sub-xeric heath forest with a high amount of dead wood, 5 Oct. 2020, leg. \& det. TH 20200024 (OULU, H), conf. MK; Ostrobottnia ultima, Tervola, Pisavaara Strict Nature Reserve, Alalaki E, UCS 7351155:3413354, on a decorticated piece of Pinus sylvestris on the ground (diam. $10 \mathrm{~cm}$, decay stage 2), in a mountain boulder field, 11 Sept. 2020, leg. \& det. MK 45/20 (OULU)

Tubulicrinis globisporus K.H. Larss. \& Hjortstam

(Fig. 55)

Distribution. New to 3 b (Fig. 1).

Notes. $4^{\text {th }}-6^{\text {th }}$ records in Finland; previous records: Padasjoki (2a), Inari (4c; two sites) (Kotiranta et al. 2009; Kunttu et al. 2019).

Specimens examined. Ostrobottnia kajanensis, Puolanka, Murtiovaara, UCS 7182:3530, on a fallen trunk of Pinus sylvestris (decay stage 4 ), in a spruce-dominated mesic heath forest, 16 Aug. 2020, leg. \& det. AM 289 (OULU), conf. MK; Kuhmo, Ulvinsalo, UCS 7102496:3662822, on a fallen trunk of Pinus sylvestris (diam. $40 \mathrm{~cm}$, decay stage 4 , kelo tree) in an old pine-dominated sub-xeric heath forest with a high amount of dead wood, 5 Oct. 2020, leg. \& det. TH 20200025 (OULU, H); Kuhmo, Elimyssalo, UCS 7129656:3664650, on a fallen trunk of Pinus sylvestris (diam. $20 \mathrm{~cm}$, decay stage 4, kelo tree) in an old pine-dominated sub-xeric heath forest, 9 Oct. 2020, leg. \& det. TH $20200034(\mathrm{H})$, and at the same site UCS 7129639:3664585, on a fallen trunk of Pinus sylvestris (diam. $40 \mathrm{~cm}$, decay stage 4, kelo tree) in an old old pine-dominated sub-xeric heath forest, 9 Oct. 2020, leg. \& det. TH 20200075 (OULU).

Tubulicrinis inornatus (H.S. Jacks. \& D.P. Rogers) Donk

(Fig. 56)

Notes. $7^{\text {th }}$ record in Finland; previous records: Lammi (2a), Padasjoki (2a), Pieksämäki (2b), Saarijärvi (3a),

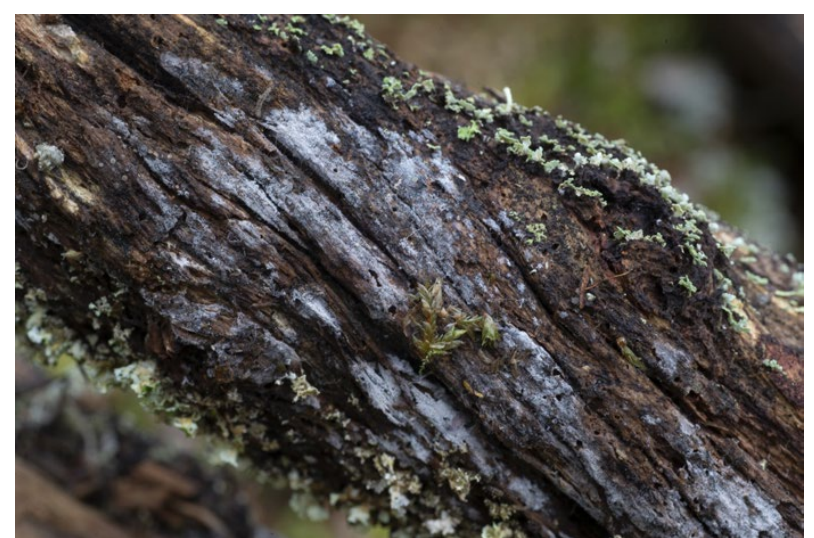

Figure 54. Tubulicrinis effugiens in Kuhmo (TH 20200024). Photo: Teppo Helo.

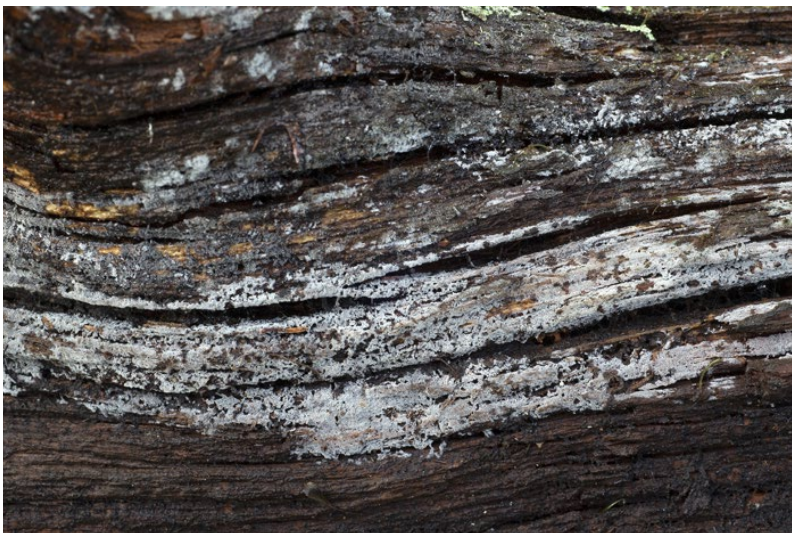

Figure 55. Tubulicrinis globisporus in Kuhmo (TH 20200034). Photo: Teppo Helo.

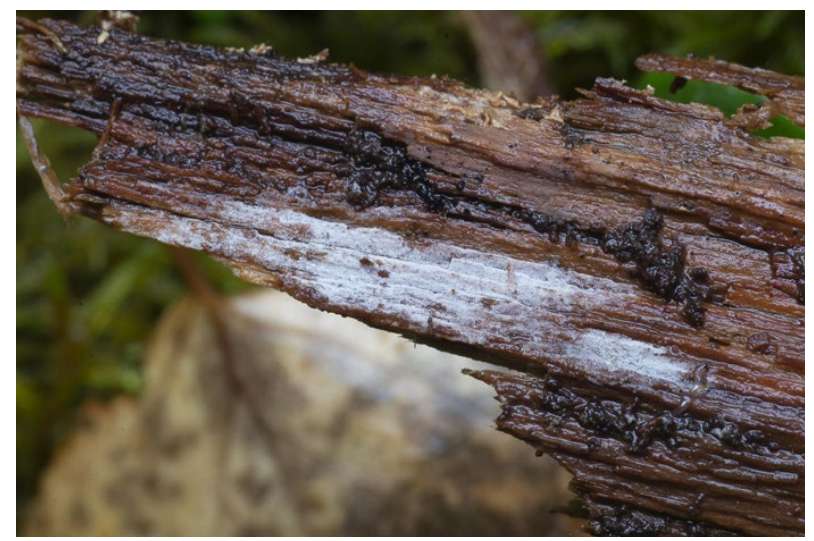

Figure 56. Tubulicrinis inornatus in Kuhmo (TH 20200076). Photo: Teppo Helo.

Kuhmo (3b), and Rovaniemi (3c) (Kotiranta et al. 2009; FinBIF 2021c).

Specimen examined. Ostrobottnia kajanensis, Kuhmo, Elimyssalo, UCS 7128689:3664898, on a fallen trunk of Pinus sylvestris (diam. $40 \mathrm{~cm}$, decay stage 3 , kelo tree) in an old spruce-dominated mesic heath forest, 10 Oct. 2020, leg. \& det. TH 20200076 (OULU), conf. MK.

Tubulicrinis propinquus (Bourdot \& Galzin) Donk (Fig. 57)

Distribution. New to 3b (Fig. 1).

Specimens examined. Ostrobottnia kajanensis, Kuhmo, 


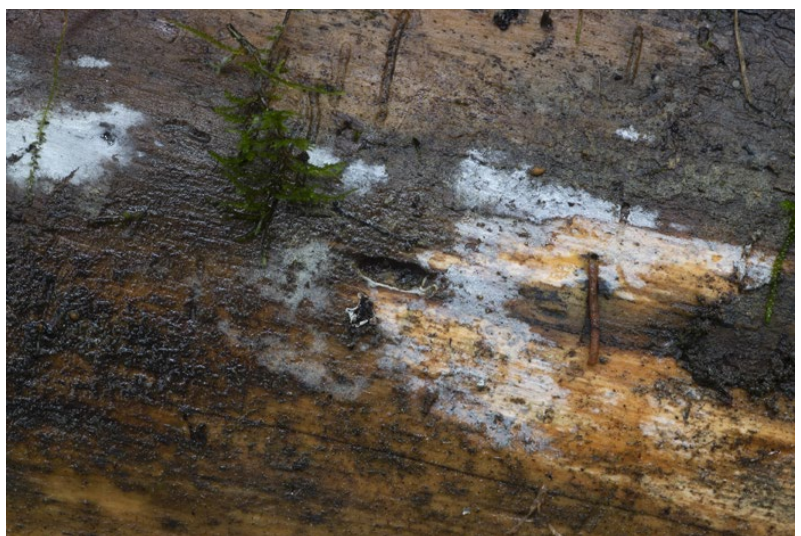

Figure 57. Tubulicrinis propinquus in Kuhmo (TH 20200032) with Botryobasidium botryosum and Sistotrema brinkmannii. Photo: Teppo Helo.

Elimyssalo, UCS 71265:36593, on a fallen trunk of Picea abies (diam. $20 \mathrm{~cm}$, decay stage 2) in an old spruce-dominated mesic heath forest, 6 Oct. 2020, leg. TH 20200032 (OULU), det. MK, and at the same site UCS 7126433:3660163, on a fallen branch of Picea abies (diam. $5 \mathrm{~cm}$, decay stage 3 ) in an old dwarf shrub spruce mire, 7 Oct. 2020, leg. TH 20200077 (OULU), det. MK.

Tulasnella allantospora Wakef. \& A. Pearson (Fig. 58)

Notes. $7^{\text {th }}-8^{\text {th }}$ records in Finland; previous records: Lohja (1b), Kirkkonummi (1b), Porvoo (2a), Muurame (2b), Sotkamo (3b), and Suomussalmi (3b) (Kotiranta et al. 2009; Kunttu et al. 2018, 2019).

Specimens examined. Ostrobottnia kajanensis, Sotkamo, Talvivaara, UCS 7093211:3557817, on a fallen trunk of Picea abies (diam. $15 \mathrm{~cm}$, decay stage 3) with Spiculogloea minuta in an old spruce-dominated mesic heath forest, 23 Sept. 2020, leg. \& det. TH 20200008 (H), conf. MK; UCS 70934:35570, on a fallen trunk of Picea abies (diam. $40 \mathrm{~cm}$, decay stage 4) with Botryobasidium botryosum in an old spruce-dominated mesic heath forest, 29 Sept. 2020, leg. \& det. TH 20200078 (OULU); Kuhmo, Elimyssalo, UCS 7130599:3662312, on a fallen trunk of Pinus sylvestris (diam. $45 \mathrm{~cm}$, decay stage 3, kelo tree) with Athelia sp. in an old pine-dominated sub-xeric heath forest, 12 Oct. 2020, leg. \& det. TH 20200079 (OULU).

Tulasnella anguifera $\mathrm{P}$. Roberts

(Fig. 59)

Distribution. New to Finland, and hence new to $3 \mathrm{~b}$ (Fig. 1).

Specimens examined. Ostrobottnia kajanensis, Kuhmo, Ulvinsalo, UCS 7102656:3662948, on a fallen trunk of Pinus sylvestris (diam. $25 \mathrm{~cm}$, decay stage 4 , kelo tree) with Athelia bombacina in a very old pine-dominated mesic heath forest, 8 Oct. 2020, leg. \& det. TH 20200080 (OULU), conf. HK, and at the same site UCS 71026:36629, on a fallen trunk of Pinus sylvestris (diam. $25 \mathrm{~cm}$, decay stage 4 , kelo tree) with Athelia bombacina, Sistotremastrum suecicum, Tulasnella permacra and Tulasnella albida in an old pine-dominated mesic heath forest, 8 Oct. 2020, leg. \& det. TH 20200081 (OULU).

\section{Tulasnella bourdotii Jülich}

(Fig. 60)

Notes. $2^{\text {nd }}-5^{\text {th }}$ records in Finland; previous record: Lieksa (3b) (Kotiranta et al. 2009).

Specimens examined. Ostrobottnia kajanensis, Sotkamo, Losonvaara UCS 7107282:3546032, on a fallen branch of Pinus

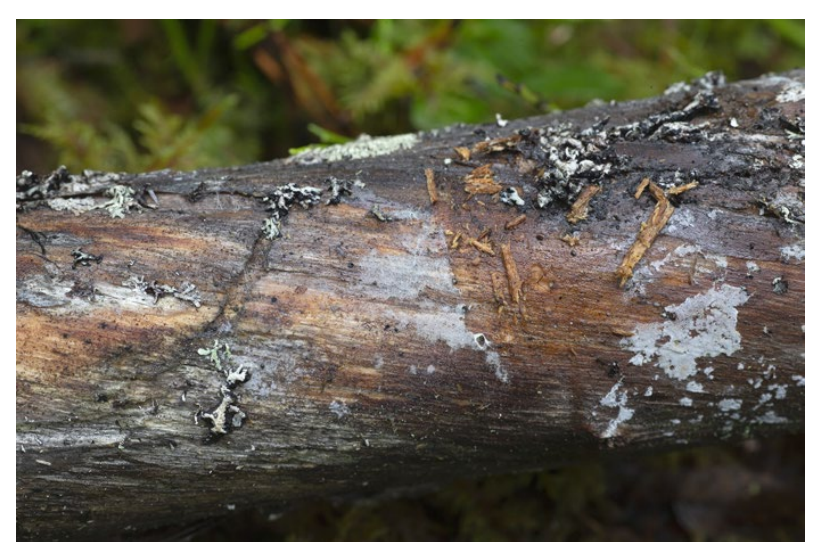

Figure 58. Tulasnella allantospora in Kuhmo (TH 20200079). Photo: Teppo Helo.

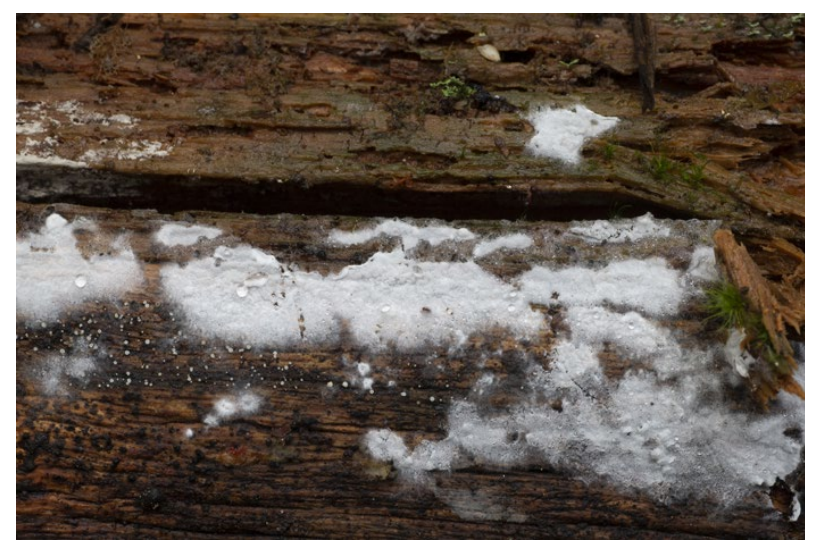

Figure 59. Tulasnella anguifera in Kuhmo (TH 20200081) with Athelia bombacina. Photo: Teppo Helo.

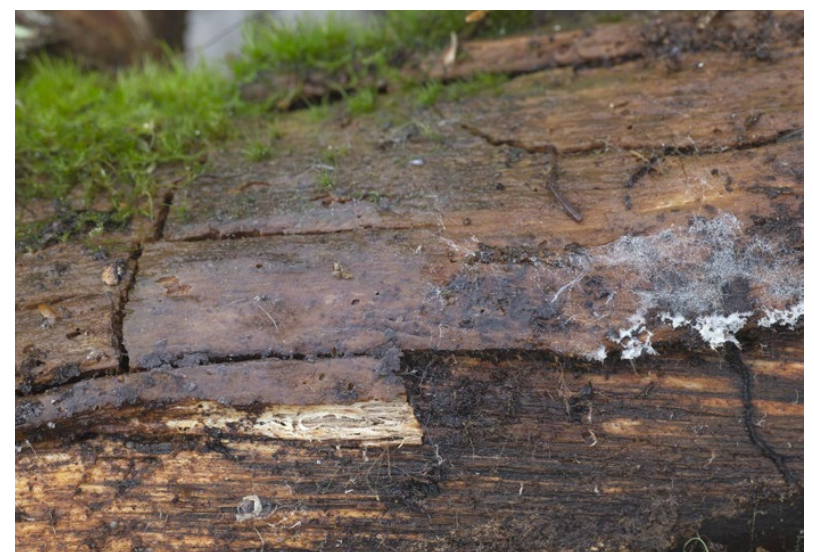

Figure 60. Tulasnella bourdotii in Kuhmo (TH 20200086). Photo: Teppo Helo.

sylvestris (diam. $5 \mathrm{~cm}$, decay stage 4) in an old spruce-dominated mesic heath forest, 28 Sept. 2020, leg. \& det. TH 20200082 (OULU), conf. MK; Sotkamo, Talvivaara UCS 70934:35570, on a fallen trunk of Picea abies (diam. $35 \mathrm{~cm}$, decay stage 4) in an old spruce-dominated mesic heath forest, 29 Sept. 2020, leg. \& det. TH 20200083 (OULU); UCS 7093442:3557179, on a fallen trunk of Picea abies (diam. $15 \mathrm{~cm}$, decay stage 3) in an old spruce-dominated mesic heath forest, 29 Sept. 2020, leg. \& det. TH 20200084 (OULU); Kuhmo, Ulvinsalo, UCS 7101930:3662971, on a fallen trunk of Pinus sylvestris (diam. $50 \mathrm{~cm}$, decay stage 4, kelo tree) with Tubulicrinis accedens in a very old spruce-dominated mesic heath forest, 5 Oct. 2020, leg. \& det. TH 20200085 (OULU), conf. MK; UCS 
7102630:3663068, on a fallen trunk of Pinus sylvestris (diam. $10 \mathrm{~cm}$, decay stage 4 , kelo tree) in a very old pine-dominated mesic heath forest, 8 Oct. 2020, leg. \& det. TH 20200086 (OULU); Kuhmo, Elimyssalo, UCS 7129321:3665284, on a fallen trunk of Picea abies (diam. $20 \mathrm{~cm}$, decay stage 3 ) in an old pine-dominated sub-xeric heath forest, 10 Oct. 2020, leg. \& det. TH 20200087 (OULU).

\section{Tulasnella brinkmannii s.1. Bres.}

(Fig. 61)

Notes. $5^{\text {th }}$ record in Finland; previous records: Suonenjoki (2b), Ristijärvi (3b), Sotkamo (3b), and Suomussalmi (3b) (Kunttu et al. 2018, 2019).

Specimen examined. Ostrobottnia kajanensis, Kuhmo, Elimyssalo, UCS 71265:36593, on a fallen trunk of Picea abies (diam. $12 \mathrm{~cm}$, decay stage 3) in an old spruce-dominated mesic heath forest, 6 Oct. 2020, leg. TH 20200089 (OULU), det. MK.

Tulasnella cystidiophora Höhn. \& Litsch.

(Fig. 62)

Distribution. New to 2 b, $3 b$ (Fig. 1).

Notes. $2^{\text {nd }}-3^{\text {rd }}$ records in Finland; previous record: Tammela (2a) (Kotiranta et al. 2009).

Specimens examined. Tavastia borealis, Rautalampi, Etelä-Konnevesi National Park, UCS 6941550:3484986, on a fallen decorticated trunk of Betula sp. (diam. $18 \mathrm{~cm}$, decay stage 2), in a mixed old-growth forest, 20 Sept. 2015 leg. \& det. J. Purhonen 6603 (JYV), det. MK conf. HK; Ostrobottnia kajanensis, Kuhmo, Elimyssalo, UCS 7126591:3660135, on a fallen trunk of Populus tremula (diam. $27 \mathrm{~cm}$, decay stage 2) in an old pine-dominated sub-xeric heath forest, 7 Oct. 2020, leg. TH 20200090 (OULU), det. MK.

\section{Tulasnella deliquescens (Juel) Juel}

(Fig. 63)

Notes. $7^{\text {th }}-10^{\text {th }}$ records in Finland; previous records: Helsinki (1b), Jyväskylä (2b), Puolanka (3b), Hyrynsalmi (3b), Suomussalmi (3b), and Sodankylä (4c) (Kotiranta et al. 2009; Kunttu et al. 2018, 2019).

Specimens examined. Ostrobottnia kajanensis, Sotkamo, Talvivaara, UCS 7092997:3557674, on a fallen branch of Populus tremula (diam. $4 \mathrm{~cm}$, decay stage 2) in an old spruce-dominated mesic heath forest, 22 Sept. 2020, leg. TH 20200091 (OULU), det. MK; Sotkamo, Losonvaara, UCS 7107262:3545992, on a fallen trunk of Juniperus communis (diam. $2 \mathrm{~cm}$, decay stage 3 ) in an old spruce-dominated mesic heath forest, 28 Sept. 2020, leg. \& det. TH 20200092 (OULU); Kuhmo, Ulvinsalo, UCS 7103531:3664853, on a fallen branch of Pinus sylvestris (diam. $1 \mathrm{~cm}$, decay stage 2 , kelo tree) in a very old spruce-dominated mesic heath forest, 4 Oct. 2020, leg. \& det. TH 20200093 (OULU); UCS 7102673:3662961, on a fallen trunk of Pinus sylvestris (diam. $25 \mathrm{~cm}$, decay stage 4, kelo tree) in an old pine-dominated mesic heath forest, 8 Oct. 2020, leg. \& det. TH 20200095 (OULU); Kuhmo, Elimyssalo, UCS 7126326:3660131, on a fallen branch of Populus tremula (diam. $4 \mathrm{~cm}$, decay stage 2) in an old spruce-dominated mesic heath forest, 7 Oct. 2020, leg. TH 20200094 (OULU), det. MK; UCS 7129643:3664595, on a fallen trunk of Pinus sylvestris (diam. $25 \mathrm{~cm}$, decay stage 2, kelo tree) in an old pine-dominated sub-xeric heath forest, 9 Oct. 2020, leg. \& det. TH 20200039 (OULU); UCS 7129726:3664908, on a fallen trunk of Betula sp. (diam. $20 \mathrm{~cm}$, decay stage 1) in an old pine-dominated sub-xeric heath forest, 10 Oct. 2020, leg. \& det. TH 20200096 (OULU); UCS 7130472:3662572, on a fallen branch of Picea

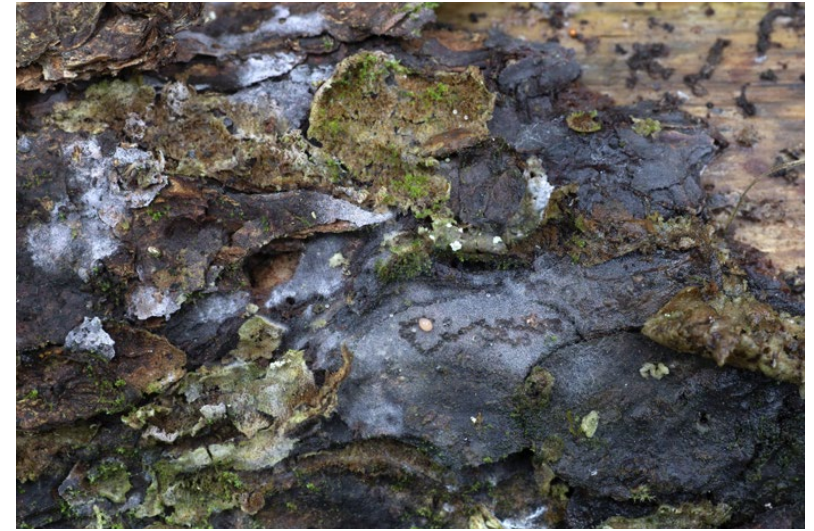

Figure 61. Tulasnella brinkmannii in Kuhmo (TH 20200089). Photo: Teppo Helo.

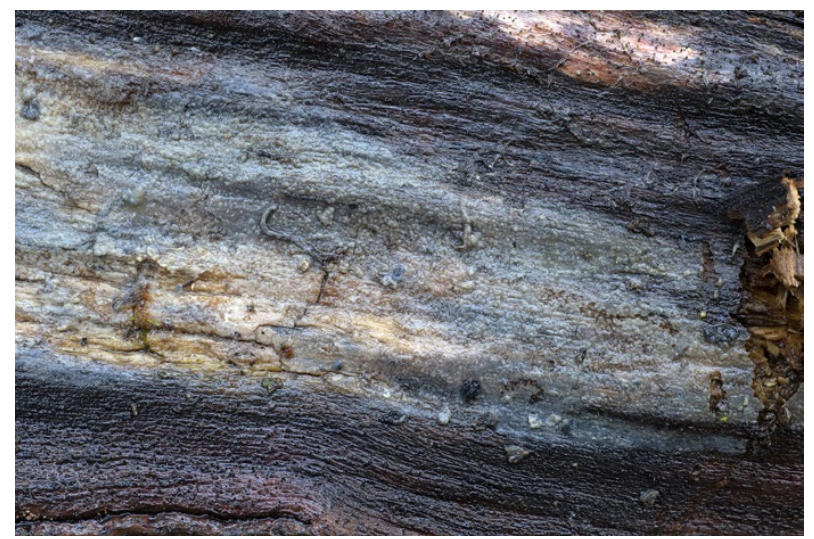

Figure 62. Tulasnella cystidiophora in Kuhmo (TH 20200090). Photo: Teppo Helo.

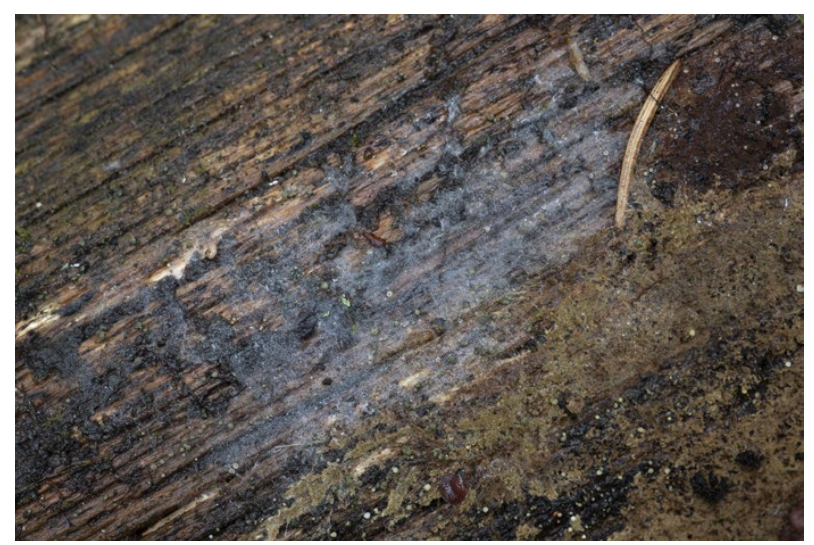

Figure 63. Tulasnella deliquescens in Kuhmo (TH 20200095) with Coniophora olivacea. Photo: Teppo Helo.

abies (diam. $1 \mathrm{~cm}$, decay stage 3 ) in an old spruce-dominated mesic heath forest, 12 Oct. 2020, leg. \& det. TH 20200097 (OULU).

\section{Tulasnella fuscoviolacea Bres.}

(Fig. 64)

Notes. $4^{\text {th }}-5^{\text {th }}$ records in Finland; previous records: Tammela (2a), Jyväskylä (2b), and Sotkamo (3b) (Kotiranta et al. 2009; Kunttu et al. 2018, 2019).

Specimens examined. Ostrobottnia kajanensis, Sotkamo, Talvivaara, UCS 71093368:3556912, on a fallen branch of Picea abies (diam. $1 \mathrm{~cm}$, decay stage 2) in an old spruce-dominated mesic heath forest, 29 Sept. 2020, leg. \& det. TH 20200098 


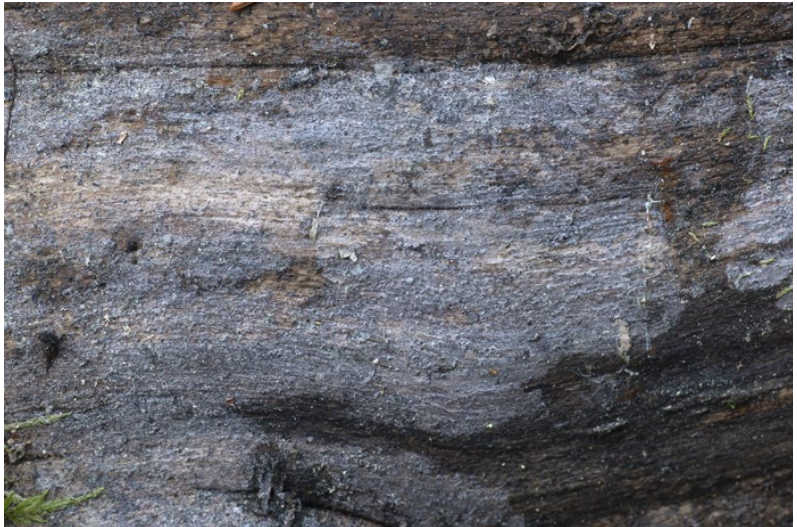

Figure 64. Tulasnella fuscoviolacea in Kuhmo (TH 20200100). Photo: Teppo Helo.

(OULU), and at the same site UCS 71093368:3556912, on a fallen branch of Picea abies (diam. $1.5 \mathrm{~cm}$, decay stage 2) in an old spruce-dominated mesic heath forest, 29 Sept. 2020, leg. \& det. TH 20200099 (OULU); Kuhmo, Ulvinsalo, UCS 7103781:3665067, on a fallen trunk of Populus tremula (diam. $35 \mathrm{~cm}$, decay stage 3 ) in a very old spruce-dominated mesic heath forest, 2 Oct. 2020, leg. \& det. TH 20200100 (OULU), and at the same site UCS 7103759:3665054, on a fallen trunk of Populus tremula (diam. $25 \mathrm{~cm}$, decay stage 2) in a very old spruce-dominated mesic heath forest, 2 Oct. 2020, leg. \& det. TH 20200101 (OULU).

\section{Tulasnella interrogans $\mathrm{P}$. Roberts}

Distribution. New to Finland, and hence new to $3 \mathrm{~b}$ (Fig. 1).

Specimen examined. Ostrobottnia kajanensis, Kuhmo, Ulvinsalo, UCS 7102582:3662587, on a fallen branch of Pinus sylvestris (diam. $1 \mathrm{~cm}$, decay stage 3, kelo tree) with Athelia acrospora in a middle-aged pine-dominated mesic heath forest with a high amount of dead wood of the previous tree generation, 8 Oct. 2020, leg. \& det. TH 20200102 (OULU), conf. MK \& HK.

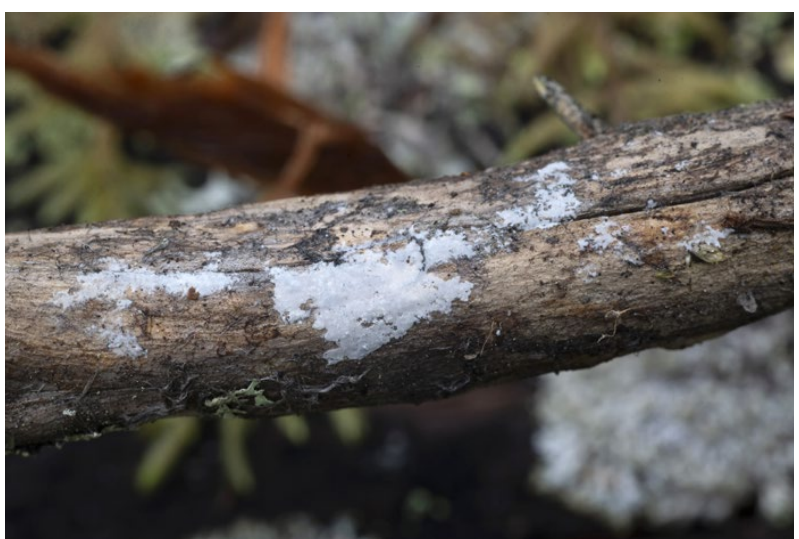

Figure 65. Tulasnella interrogans in Kuhmo (TH 20200102) with Athelia acrospora. Photo: Teppo Helo.

\section{Tulasnella pallida Bres.}

(Fig. 66)

Distribution. New to 3a (Fig. 1).

Notes. $5^{\text {th }}-7^{\text {th }}$ records in Finland; previous records: Marttila (1b), Ylöjärvi (2b), Sotkamo (3b), and Puolanka (3b) (Kotiranta et al. 2009; Kunttu et al. 2020).
Specimens examined. Ostrobottnia ouluensis, Oulu, Iinatti, UCS 7209158:3434103, on a stump of Salix caprea (decay stage 3), in a small pine-dominated mesic heath forest, 6 May 2020, leg. \& det. AM 276 (OULU), conf. MK; Ostrobottnia kajanensis, Kuhmo, Elimyssalo, UCS 71265:36593, on a fallen trunk of Picea abies (diam. $10 \mathrm{~cm}$, decay stage 1) with Luellia recondita and Sistotrema autumnale in an old spruce-dominated mesic heath forest, 6 Oct. 2020, leg. TH 20200103 (OULU), det. MK and at the same site UCS 7128711:3665035, on a fallen trunk of Picea abies (diam. $10 \mathrm{~cm}$, decay stage 3 ) in an old

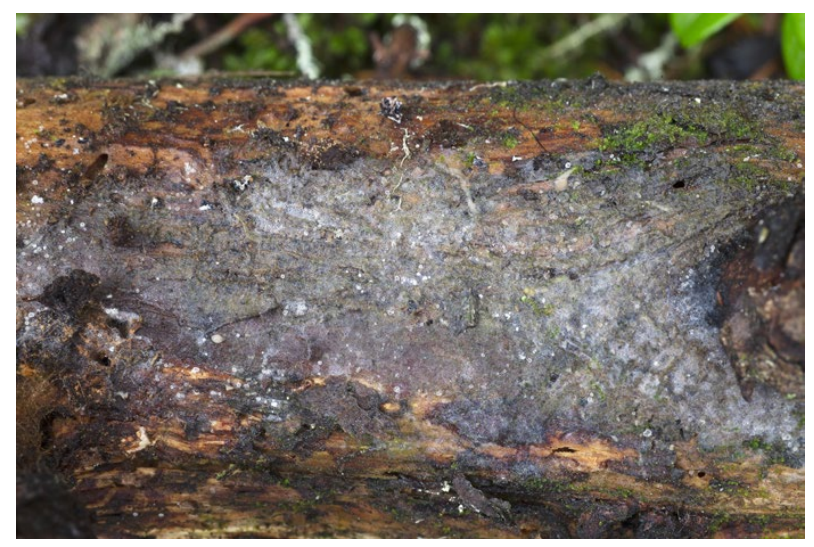

Figure 66. Tulasnella pallida in Kuhmo (TH 20200105). Photo: Teppo Helo.

spruce-dominated mesic heath forest, 10 Oct. 2020, leg. \& det. TH 20200105 (OULU), conf. MK; Kuhmo, Ulvinsalo, UCS 7102677:3662983, on a fallen trunk of Pinus sylvestris (diam. $8 \mathrm{~cm}$, decay stage 2) with Tubulicrinis subulatus and Xylodon brevisetus in a very old pine-dominated mesic heath forest, 8 Oct. 2020, leg. \& det. TH 20200104 (OULU).

\section{Tulasnella permacra P. Roberts}

Notes. $6^{\text {th }}-8^{\text {th }}$ records in Finland; previous records: Helsinki (1b), Tammisaari (1b), Sotkamo (3b; two sites), and Puolanka (3b) (Kotiranta et al. 2009; Kunttu et al. 2018, 2019, 2020).

Specimens examined. Ostrobottnia kajanensis, Sotkamo, Losonvaara, UCS 7107510:3545128, on a fallen trunk of Picea abies (diam. $25 \mathrm{~cm}$, decay stage 2) with Coniophora olivacea, Tubulicrinis strangulatus and Tylospora fibrillosa in an old spruce-dominated mesic heath forest, 7 Sept. 2020, leg. \& det. TH 20200107 (OULU), and at the same site UCS 7107559:3545188, on a fallen trunk of Populus tremula (diam. $10 \mathrm{~cm}$, decay stage 3) with Athelia epiphylla in an old spruce-dominated mesic heath forest, 8 Sept. 2020, leg. \& det. TH 20200108 (OULU); Kuhmo, Elimyssalo, UCS 7129534:3664519, on a fallen trunk of Betula sp. (diam. $15 \mathrm{~cm}$, decay stage 4) with Piloderma byssinum and Amphinema byssoides in an old spruce-dominated mesic heath forest, 9 Oct. 2020, leg. \& det. TH 20200111 (OULU); Kuhmo, Ulvinsalo, UCS 71026:36629, on a fallen trunk of Pinus sylvestris (diam. $25 \mathrm{~cm}$, decay stage 4, kelo tree) with Athelia bombacina, Sistotremastrum suecicum, Tulasnella anguifera and Tulasnella albida in an old pine-dominated mesic heath forest, 8 Oct. 2020, leg. \& det. TH 20200081 (OULU); UCS 71026:36629, on a fallen trunk of Pinus sylvestris (diam. $23 \mathrm{~cm}$, decay stage 3, kelo tree) with Tylospora fibrillosa in a very old pine-dominated mesic heath forest, 8 Oct. 2020, leg. \& det. TH 20200110 (OULU). 


\section{Tulasnella thelephorea (Juel) Juel}

Notes. $7^{\text {th }}$ record in Finland; previous records: Lohja (1b), Inkoo (1b), Kajaani (3b; two sites), Lieksa (3b), and Utsjoki (4d) (Kotiranta et al. 2009; Kunttu et al. 2018, 2019).

Specimen examined. Ostrobottnia kajanensis, Kuhmo, Ulvinsalo, UCS 7103786:3665057, on a fallen trunk of Betula sp. (diam. $20 \mathrm{~cm}$, decay stage 3) with Athelia fibulata in a very old spruce-dominated mesic heath forest, 2 Oct. 2020, leg. \& det. TH 20200112 (OULU), conf. MK.

Vararia racemosa (Burt) Rog. \& Jacks. ssp. lapponica Hallenberg

(Fig. 67)

Distribution. New to $3 \mathrm{~b}$ (Fig. 1).

Notes. $2^{\text {nd }}$ record in Finland; previous record: Salla (4c) (Kotiranta et al. 2009). Data Deficient.

Specimen examined. Ostrobottnia kajanensis, Kuhmo, Elimyssalo, UCS 71265:36593, on a fallen trunk of Picea abies (diam. $45 \mathrm{~cm}$, decay stage 3) with Tomentella stuposa, Xylodon brevisetus s.str. and Botryobasidium subcoronatum in an old spruce-dominated mesic heath forest, 6 Oct. 2020, leg. TH 20200031 (OULU), det. MK.

\section{Xenasma pruinosum (Pat.) Donk}

(Fig. 68)

Distribution. New to 2a (Fig. 1).

Notes. $4^{\text {th }}$ record in Finland; previous records: Luhanka (2b), Jyväskylä (2b), and Kajaani (3b) (Kotiranta et al. 2009; Kunttu et al. 2018).

Specimen examined. Tavastia australis, Kuhmoinen, Kissakulma, Vierula UCS 6823647:3405308, on a fallen branch of Salix caprea (diam. $10 \mathrm{~cm}$, decay stage 2) in a summer house garden, 22 Sept. 2019, leg. \& det. JP 4170 (H), conf. HK.

\section{Xenasmatella borealis (K.H. Larss. \& Hjortstam)} Duhem

Notes. $9^{\text {th }}-10^{\text {th }}$ records in Finland; previous records: Helsinki (1b), Kuhmoinen (2b), Petäjävesi (2b), Kajaani (3b), Lieksa (3b), Puolanka (3b), Sodankylä (4c), and Salla (4c) (Kotiranta et al. 2009; Kunttu et al. 2018, 2020).

Specimens examined. Ostrobottnia kajanensis, Kuhmo, Ulvinsalo, UCS 7103599:3665407, on a fallen trunk of Pinus sylvestris (diam. $4 \mathrm{~cm}$, decay stage 3 , kelo tree) in a very old spruce-dominated mesic heath forest, 13 Oct. 2020, leg. \& det. TH 20200121 (OULU), and at the same site UCS 7102648:3662954, on a fallen trunk of Pinus sylvestris (diam. $6 \mathrm{~cm}$, decay stage 3, kelo tree) in a very old pine-dominated mesic heath forest, 8 Oct. 2020, leg. \& det. TH 20200117 (OULU); Kuhmo, Elimyssalo, UCS 7130440:3662325, on a fallen branch of Pinus sylvestris (diam. $6 \mathrm{~cm}$, decay stage 2, kelo tree) in an old pine-dominated sub-xeric heath forest, 12 Oct. 2020, leg. \& det. TH 20200119 (OULU), and at the same site UCS 71265:36593, on a fallen trunk of Juniperus communis (diam. $3 \mathrm{~cm}$, decay stage 3 ) in an old spruce-dominated mesic heath forest, 6 Oct. 2020, leg. TH 20200116 (OULU), det. MK.

Xylodon borealis (Kotir. \& Saaren.) Hjortstam \& Ryvarden Distribution. New to 3a (Fig. 1).

Specimen examined. Ostrobottnia ouluensis, Pudasjärvi, Sarvisuo, UCS 7209158:3434103, on a fallen trunk of Populus tremula,

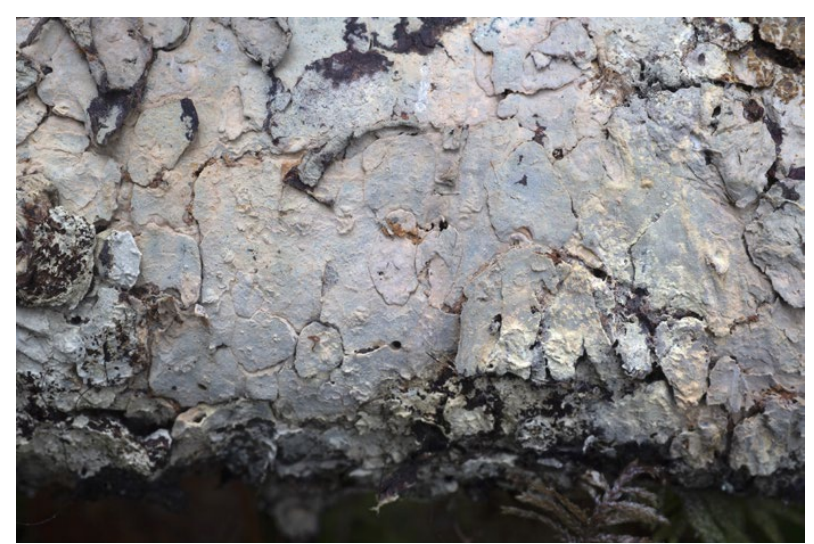

Figure 67. Vararia racemosa in Kuhmo (TH 20200031). Photo: Teppo Helo.

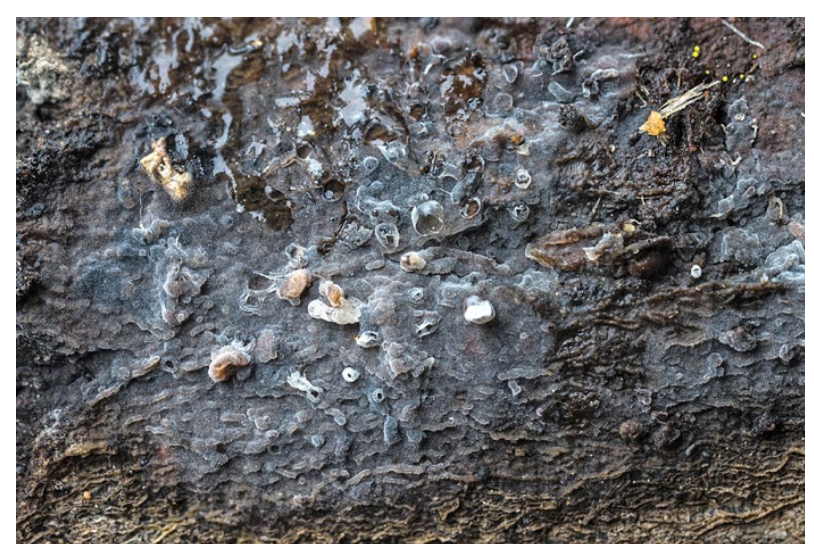

Figure 68. Xenasma pruinosum in Kuhmoinen (JP 4170). Photo: Jorma Pennanen.

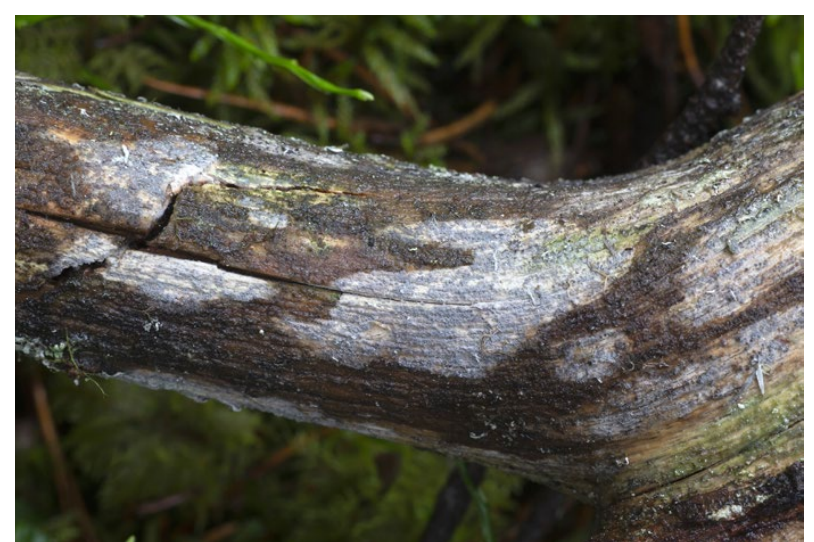

Figure 69. Xenasmatella borealis in Kuhmo (TH 20200119). Photo: Teppo Helo.

in a spruce-dominated herb-rich forest next to a stream running from a spring, 11 July 2018, leg. AM 66-18 (OULU), det. MK.

\section{Discussion}

Species new to Finland

Hyphoderma lapponicum has been reported from Sweden, Norway, Spain, and one in unpublished (H. Kotiranta) record from Russian Far East (Eriksson \& Ryvarden 1975; GBIF Secretariat 2019). The ecology of the species seems to be poorly known, and its delimitation against $H$. obtusiforme deserves further study. 
Mycostilla vermiformis is distributed in temperate and boreal forests of Europe, and it has been found in Sweden, Norway, Denmark, Russia, Poland, Czech Republic, United Kingdom, and France (see Spirin et al. 2019b). The hosts described by Spirin et al. (2019b) were Picea abies and Pinus sylvestris. In Finland, basidiomata grew on coarse trunks of intermediately decayed Picea abies. The site in Helsinki is now partly destroyed. Previous reports of Stypella vermiformis in Finland represent another species. For the taxonomy of the complex see Spirin et al. (2019b).

Proterochaete adusta (Alvarenga et al. 2019) has been collected from USA (Idaho), Canada (Alberta, British Columbia), China (Jilin), Russia (Primorie, Khabarovsk), Norway (Møre and Romsdal) (Alvarenga et al. 2019), Germany (Thuringenia, Saxony, North Rhine-Westphalia) (Rödel et al. 2020) and now Finland (Kainuu, South Häme).

In the USA, P. adusta grew on a decorticated Populus trichocarpa trunk (Burt 1915), in Canada on Populus trichocarpa and Acer negundo (Martin 1953; Alvarenga et al. 2019), in Russia on an undescribed deciduous tree and a Betula pseudosieboldianum (Wells \& Raitviir 1966; Alvarenga et al. 2019), in Norway on an undescribed hardwood (Alvarenga et al. 2019), in Germany on fallen Populus trunks (five records), inside a rotten Populus stump (one record), and on a fallen Quercus trunk (one record) (Rödel et al. 2020), and in Finland on the bark of a fallen and hollow Populus tremula trunk (2017 record). There is no description of the substrate in the 1964 Finnish record. In summary, P. adusta seems to demand, or at least to prefer, deciduous trees, especially Populus as its substrate (Rödel et al. 2020).

With the exception of substrate, the ecology of P. adusta remains unrecorded. Rödel et al. (2020) report that one of their specimens grew on an eastern bank of a river. The 2017 Finnish record grew on a very old Picea abies dominated thin-peated Vaccinium myrtillus - Vaccinium vitis-idaea spruce mire with a substantial amount of dead wood. Populus tremula and Salix caprea were secondary species in the forest. There is no description of the ecology in the 1964 Finnish record.

The fresh basidioma from the 2017 Finnish record was smooth - finely tuberculate, very thin and almost transparent. A cross section of the dry basidioma was a minimum of $20-40 \mu \mathrm{m}$ compared to the cross sections 100-300 $\mu \mathrm{m}$ (Alvarenga et al. 2019) or 110-220 $\mu \mathrm{m}$ (Rödel et al. 2020). The dry basidioma of the 1964 Finnish record is rather finely grandinoid - finely tuberculate.

Pseudotomentella alobata has been reported in Sweden, Norway and Slovenia (Svantesson et al. 2019). To date, data on habitat are scarce, but recent Scandinavian collections have been made in old-growth coniferous or mixed forests on soil with high $\mathrm{pH}$ (Svantesson et al. 2019). The species is described from the Pseudotomentella tristis group. Most of the $P$. tristis specimens in the collections are incorrectly identified, so the distribution of the species is not reliably known. It is probably quite rare.

According to GBIF Secretariat (2019), Pseudoxenasma verrucisporum is known in Sweden, Norway and
Austria. Eriksson et al. (1981) report that this species typically inhabits conifer branches in closed, moist forests, often growing together with Globulicium hiemale, as in the case of the Finnish collection. In a Nordic context, the species appears to be southern (most finds are from the hemiboreal zone), although it may extend further north along the seacoast. The species has not been found in Russia, which would indicate that it may have an oceanic distribution (V. Spirin, pers. comm.). Most collections have been made close to the coast, where it can be locally common. Overall, the species appears to be rather rare. The collection from Helsinki fits well with this general pattern, as it was found in an old, closed spruce forest on the southern coast of Finland.

Sistotrema subtrigonospermum is a globally distributed species: records have been reported in several countries in Europe, Africa, North and South America, India, and Polynesia (see Martini 2021a). Typical substrata include advanced decayed lying trunks and fallen branches of deciduous trees (e.g., Fagus, Quercus, Populus, Salix), but there are collections from coniferous tree too (see Martini 2021a). The majority of substrata have been advanced or strongly decayed. The basidiomata in Finland inhabited fallen thin and decayed branches of deciduous trees and Picea abies.

Spiculogloea minuta has been reported in France, Germany, Norway, Russia, and United Kingdom (GBIF Secretariat 2019; Roberts 1997; Trichies 2002; Rödel 2014; Spirin 2016; Spirin 2019b). The known substrata of this parasite species are Helicogloea lagerheimii, Hyphoderma argillaceum, Mycostilla vermiformis, Phanerochaete sordida, Tubulicrinis accedens, Tulasnella allantospora, T. eichleriana, T. saveloides, T. tomaculum and T. violea (Roberts 1997; Trichies 2002; Rödel 2014; Spirin 2016; Spirin 2019b). The Finnish specimen was collected in association with Tulasnella allantospora on a decayed Picea abies trunk.

Tomentella botryoides is a widely distributed species that has been found in many countries in Europe, as well as in Morocco, Caucasus, Russian Far East, India and North America (see Martini 2021b). The collections have been made both from deciduous (e.g., genera Quercus, Fagus, Betula) and coniferous trees (e.g., genera Thuja, Abies, Pinus, Pseudostuga), where the substrate were mainly intermediately or strongly decayed lying trunks and branches (see Martini 2021b).

Tomentella neobourdotii has been reported in Sweden, Estonia, Denmark, Russian Federation, Belarus, Germany, Czech Republic, Austria, Switzerland, Liechtenstein, France, Italy, Spain, Portugal, Macedonia, Georgia, Iran and the USA (GBIF Secretariat 2019; Martini 2021c). It has been mainly collected from decayed trunks of deciduous trees (Martini 2021c).

Tomentella subpilosa has been reported in Denmark, Austria, France, Switzerland, Czech Republic and Slovakia (Svrček 1960; GBIF Secretariat 2019; Martini 2019d). It has been mainly collected from decayed trunks or on branches of Larix decidua, Abies alba, Pinus halepensis, P. sylvestris, Picea abies, Fagus sylvatica, Populus sp, although basidiomata have also been 
found growing on bark or hard surfaced wood (Svrček 1960; Martini 2019).

Tomentella subtestacea has a global distribution and GBIF Secretariat (2019) has listed it in Sweden, Norway, Denmark, Estonia, Russian Federation, Belarus, Ukraine, Germany, Poland, Belgium, France, Czech Republic, Hungary, United Kingdom, Spain, Azerbaijan, USA, Mexico and Laos. Ecological requirements are unknown or unreported. Previously Tomentella aff. subtestacea was reported in Finland in 1979 (Kotiranta et al. 2009), although the identity of the specimen remained unclear.

Tulasnella anguifera has previously been reported only in France and United Kingdom (Roberts 1992; GBIF Secretariat 2019; Trichies 2002) and was collected from decayed trunks or branches of Picea abies, Picea sp., and Prunus spinosa (Roberts 1992; Trichies 2002). Both Finnish specimens grew inside basidiomata of Athelia bombacina on decorticated Pinus sylvestris kelo trees. The second specimen occurred on the same trunk, in association with Sistotremastrum suecicum, Tulasnella permacra, and Tulasnella albida.

Tulasnella interrogans has been reported in Belgium, France, United Kingdom, and Spain (Roberts 1992; Dämon 2001; GBIF Secretariat 2019), where it was collected from decayed trunks or branches of Corylus sp., Picea sp., Salix sp. and inside a basidioma of Botryobasidium subcoronatum (Roberts 1992; Trichies 2002). A Finnish specimen grew under and around basidioma of Athelia bombacina on a decorticated kelo branch of Pinus sylvestris. The habitat had been thinned at some point, but substantial amounts of decaying pine wood were still present onsite from the previous tree generations.

Tulasnella is a genus whose species' ecology, biogeography, substrata and habitat requirements are poorly known. Notes on habitats in old collections are often incompletely described and hence do not allow conclusions to be drawn. Many of the Tulanella species appear to favor herb-rich forests, but some occur in very diverse biotopes and can even be found in barren places. It is possible that some Tulasnella species even prefer old-growth forests. In fact, our specimens of these two Tulasnella species were found in one of the finest natural forests in Finland. Nonetheless, the number of collections is still insufficient to draw conclusions about the ecological requirements of these species.

The red-list status of the species new to Finland has not been assessed.

\section{Species with only one previous global record}

The second global record of Caudicicola gracilis was found in Oulu, Finland. The basidioma grew on a sawn block of Betula sp. that was leaning against a tree in a rather moist spruce-dominated drained and transformed mire. The first recording of C. gracilis was in 2014-2015 in Pyhäjärvi, central Finland and was described as a new species (Kotiranta et al. 2017). The habitat was a boreal wooded, drained minerotrophic mire. Spruce was the dominant tree species intermixed with pine, birch and some willow. All the basidiomata grew on the undersides of spruce or pine stumps (roots) and could only be seen after the stumps had been lifted from the ground (Kotiranta et al. 2017). However, if this is the typical growth habit of this species, then it will be a difficult species to detect. Caudicicola gracilis appears to be a wood- or litter-decomposer, since there are no mycorrhizal species in Steccherinaceae or in Polyporales (Hibbett et al. 2014).

\section{Significant extensions of the known} distribution range

Most of the new records were found in expected regions, relatively close to their previously known distribution. Nonetheless, the records of some species were located far from their earlier finds or were found in an unexpected location. This 'expansion' of the species' distribution range can be largely explained by the increasing survey activity in different parts of Finland. The new record of Trechispora stellulata in Enontekiö is $570 \mathrm{~km}$ north from the nearest previous record in Puolanka. The new record of Ceriporia bresadolae in Helsinki extends its known range by $500 \mathrm{~km}$ to the south. However, it should be noted that collections identified as C. bresadolae in Estonia (Runnel et al. 2021), are actually closer than the previous collections in Finland (NATARC 2019). Similarly, the new records of Protodontia subgelatinosa in Enontekiö, Amaurodon cyaneus, Protomerulius brachysporus and Tomentella fuscocinerea, all in Kuhmo, were found approximately $500 \mathrm{~km}$ further north compared to previous records. Tomentellopsis zygodesmoides was found in Sotkamo, which is situated $400 \mathrm{~km}$ north of the nearest previously known location in Tuusula.

\section{Conclusions}

The occurrence and distribution of many aphyllophoroid fungal species are still poorly known in Finland, as demonstrated by this article. Our new records indicate that many species most likely do not have such a scattered distribution as suggested by previous records. Thus, there are still large gaps in our knowledge of their ecology, biogeography, substrata and habitat requirements. The reasons behind this include time-consuming sampling, small basidiomata that are difficult to find, narrow ecological niches (substratum), changing taxonomy, and challenges in species identification. For example, the fungal communities that occupy the smallest fractions of woody debris seem to be poorly known, since this substrate has been commonly overlooked. However, new records are reported frequently and, therefore, knowledge accumulates all the time. New information on aphyllophoroid fungi will be gathered when performing field surveys in poorly studied areas and neglected habitats, or on species with tiny basidiomata. More effort should be allocated into field studies to establish which species are truly rare and to determine the actual distribution range. Naturally, a certain portion of aphyllophoroid fungal species are truly rare or geographically restricted, for example, due to habitat specialization. All such additional knowledge of species occurrence and habitats is important for a deeper understanding of diversity, ecology and conservation requirements of aphyllophoroid fungi. 


\section{Acknowledgments}

We would like to thank the following people who made the collections, identified specimens, assisted with fieldwork or helped us otherwise: Peter von Bagh, Pekka Halonen, Maija-Liisa Heinonen, Pekka Heinonen, Maire Helo, Pekka Helo, Pauli Jokikokko, Sanna-Mari Kunttu, Annina Käppi, Tiina Laitinen, Hannu Lehtonen, Elia Martini, Suvi Pousi, Jenna Purhonen, Annu Ruotsalainen, Ossi Ryhänen, Pertti Salo, Viacheslav Spirin, Pekka Tolvanen, Laura Vuoksenmaa, and Henry Väre. Author PV would like to thank Esa Huhta in Natural Resources Institute LUKE for leading the project 'Demometsä' (A75831). Dr. David Wilson revised the English language.

\section{References}

Ahti, T., Hämet-Ahti, L. \& Jalas, J. 1968. Vegetation zones and their sections in northwestern Europe. Annales Botanici Fennici 5(3): 169-211.

Alvarenga, R. L. M., Spirin, V., Malysheva, V., Giberton, T. B. \& Larsson, K-H. 2019. Two new genera and six other novelties in Heterochaete sensu lato (Auriculariales, Basidiomycota). Botany 97(3): 439-451. https://doi.org/10.1139/cjb-2019-0046

von Bagh, P. 2020. Karvaorvakka Porvoossa. Sienilehti 72(2): 12.

Bernicchia, A. \& Gorjón, S. P. 2010. Corticiaceae s.l. Fungi Europaei 12. Edizioni Candusso, Alassio.

Burt, E. A. 1915. The Thelephoraceae of North America. V. Tremellodendron, Eichleriella, and Sebacina. Annals of the Missouri Botanical Garden 2: 731-770.

Dämon, W. 2001. Die corticioiden Basidienpilze des Bundeslandes Salzburg (Österreich): Floristik, Lebensräume und Substratökologie. Bibliotheca Mycologica 189: 1-413.

Eriksson, J., Hjortstam, K. \& Ryvarden, L. 1981. The Corticiaceae of North Europe 6. Phlebia-Sarcodontia. Fungiflora, Oslo.

Eriksson, J. \& Ryvarden, L. 1975. The Corticiaceae of North Europe vol. 3. Fungiflora, Oslo.

FinBIF 2021a. The list of biogeographical provinces. Finnish Biodiversity Information Facility. Available from: https:/laji.fi/theme/ emk [Cited 26 Feb. 2021].

FinBIF 2021b. Biogeographical provinces on a map. Finnish Biodiversity Information Facility. Available from https://laji.fi/map?overlayNames $=$ geobiologicalProvinces [Cited 26 Feb. 2021].

FinBIF 2021c. Species search - Browse and discover species information, descriptions and pictures. Finnish Biodiversity Information Facility. Available from https://laji.fi/en/taxon [Cited 26 Feb. 2021].

GBIF Secretariat 2019. Global Biodiversity Information Facility - Backbone Taxonomy. Checklist dataset. https://doi.org/10.15468/39omei [Cited 13 Mar. 2021]

Hansen, L. \& Knudsen, H. 1997. Nordic macromycetes 3. Heterobasidioid, aphyllophoroid and gastromycetoid Basidiomycetes. Nordsvamp, Copenhagen.

Heikinheimo, O. \& Raatikainen, M. 1981. Grid references and names of localities in the recording of biological finds in Finland. Notulae Entomologicae 61: 133-154.

Hibbett, D. S., Bauer, R., Binder, M., Giachini, A. J., Hosaka, K., Justo, A., Larsson, E., Larsson, K.-H., Lawrey, J. D., Miettinen O., Nagy, L. G., Nilsson, R. H., Weiss, M. \& Thorn, R. G. 2014. Agaricomycetes. In: McLaughlin, D. J. \& Spatafora, J. W. (eds), Systematics and evolution: 373-429. Springer, Berlin \& Heidelberg.

Hjortstam, K. \& Ryvarden, L. 2009. A checklist of names in Hyphodontia sensu stricto - sensu lato and Schizopora with new combinations in Lagarobasidium, Lyomyces, Kneiffiella, Schizopora and Xylodon. Synopsis Fungorum 26: 33-55.

Jokikokko, P. 2020. Suomesta löytyi jo toinen loukkokäävän esiintymä. Sienilehti 72(4): 8-9.
Juutilainen, K. 2016. Ecology, environmental requirements and conservation of corticioid fungi occupying small diameter dead wood. Jyväskylä Studies in Biological and Environmental Science 313: $1-55$.

Knudsen, H. \& Vesterholt, J. (eds). 2008. Funga Nordica. Agaricoid, boletoid and cyphelloid genera. Nordsvamp, Copenhagen.

Kontula, T. \& Raunio, A. (eds) 2018. Threatened habitat types in Finland 2018. Red List of habitats. Part II: Descriptions of habitat types. The Finnish Environment 5/2018. Finnish Environment Institute and Ministry of the Environment.

Korhonen Kari, T. (ed.) 2009. VMI11 Maastotyöohje 2009. Koko Suomi. Metsäntutkimuslaitos, Valtakunnan metsien inventointi VMI, Joensuu. http://urn.fi/URN:NBN:fi-fe201603038534 [Cited 1 Jul. 2021]

Kotiranta, H. \& Shiryaev, A. 2013. Notes on Aphyllophoroid fungi (Basidiomycota) in Kevo, collected in 2009. Kevo Notes 14: 1-22.

Kotiranta, H., Saarenoksa, R. \& Kytövuori, I. 2009: Aphyllophoroid fungi of Finland. A check-list with ecology, distribution, and threat categories. Norrlinia 19: 1-223.

Kotiranta, H., Kulju, M. \& Miettinen, O. 2017. Caudicicola gracilis (Polyporales, Basidiomycota), a new polypore species and genus from Finland. Annales Botanici Fennici 54: 159-167.

Kotiranta, H., Junninen, J., Halme, P., Kytövuori, I., von Bonsdorff, T., Niskanen, T. \& Liimatainen, K. 2019. Aphyllophoroid fungi. In: Hyvärinen, E., Juslén, A., Kemppainen, E., Uddström, A. \& Liukko, U.-M. (eds). The 2019 Red List of Finnish Species, pp. 234-247. Ministry of the Environment \& Finnish Environment Institute, Helsinki.

Kunttu, P., Helo, T., Kulju, M., Julkunen, J. \& Kotiranta, H. 2020. Contributions to the knowledge of aphyllophoroid and heterobasidioid funga (Basidiomycota) in Finland. Karstenia 58(1): 118-143. https://doi.org/10.29205/ka.2020.490

Kunttu, P., Helo, T., Kulju, M., Julkunen, J., Pennanen, J., Shiryaev, A. G., Lehtonen, H. \& Kotiranta, H. 2019. Aphyllophoroid funga (Basidiomycota) of Finland: range extensions and records of nationally new and rare species. Acta Mycologica 54(2): 1128. https:// doi.org/10.5586/am. 1128

Kunttu, P., Juutilainen, K., Helo, T., Kulju, M., Kekki, T. \& Kotiranta, H. 2018. Updates to Finnish aphyllophoroid funga (Basidiomycota): new species and range extensions. Mycosphere 9(3): 519-564. https://doi.org/10.5943/mycosphere/9/3/7

Kunttu, P., Kulju, M., Kekki, T., Pennanen, J., Savola, K., Helo, T. \& Kotiranta, H. 2016. Extensions of known geographic distribution of aphyllophoroid fungi (Basidiomycota) in Finland. Mycosphere 7: 333-357. https://doi.org/10.5943/mycosphere/7/3/7

Kunttu, P., Kulju, M. \& Kotiranta, H. 2012. New national and regional biological records for Finland 2. Contributions to the Finnish aphyllophoroid funga (Basidiomycota). Memoranda Societas pro Fauna et Flora Fennica 88: 61-66.

Kunttu, P., Pennanen, J., Helo, T., Kulju, M. \& Söderholm, U. 2013. New national and regional biological records for Finland 4. Additions to the knowledge of Finnish aphyllophoroid funga (Basidiomycota). Memoranda Societas pro Fauna et Flora Fennica 89: 119-124.

Kunttu, P., Pennanen, J., Kulju, M., Kekki, T. \& Suominen, M. 2014. Noteworthy records of aphyllophoroid fungi in Finland (Basidiomycota). Acta Mycologica 49: 221-235. https://doi.org/10.5586/ am.2014.017

Leikola, M. 1969. On the termination of diameter growth of Scots pine in old age in northernmost Finnish Lapland. Silva Fennica 3: 50-61.

Malysheva, V., Spirin, V., Schoutteten, N., De Lange, R., Pennanen, J. \& Larsson, K.-H. 2020. New and Noteworthy Species of Helicogloea (Atractiellomycetes, Basidiomycota) from Europe. Annales Botanici Fennici 57: 1-7. https://doi.org/10.5735/085.057.0101

Martin, G. T. 1953. New species of Protodontia from British Columbia. Journal of the Washington Academy of Sciences 43(1): 16-18.

Martini, E. 2021a. Species with descriptions, images and drawings: Sistotrema subtrigonospermum D.P. Rogers (1935). https://www. aphyllo.net/spec.php?id=804300 [Cited 26 Mar. 2021] 
Martini, E. 2021b: Species with descriptions, images and drawings: Tomentella botryoides (Schwein.) Bourdot \& Galzin (1924). https:// www.aphyllo.net/spec.php?id=1311400 [Cited 26 Mar. 2021]

Martini, E. 2021c. Species with descriptions, images and drawings: Tomentella neobourdotii M.J. Larsen (1968). https://www.aphyllo. net/spec.php?id=246100 [Cited 27 Mar. 2021]

Martini, E. 2019d. Tomentella subpilosa Litsch. Excerpts from Crusts \& Jells - Descriptions and reports of resupinate Aphyllophorales and Heterobasidiomycetes. No 137. https:/www.aphyllo.net/excerpts/ ecj137_Tomentella-subpilosa.pdf

Miettinen, O. 2012. The Corticiaceae survey of the forests in Veräjämäki, Patola and Tali 2011. Publications by City of Helsinki Environment Centre 6/2012: 1-24.

NATARC 2019. Database of Estonian fungi collections. Natural history archives and information network (NATARC). https://natarc.ut.ee/ en/seenekogud.php [Cited 10 Apr. 2021]

Niemelä, T. 2016. The polypores of Finland. Norrlinia 31: 1-430.

Niemelä, T., Wallenius, T. \& Kotiranta, H. 2002. The kelo tree, a vanishing substrate of specified wood-inhabiting fungi. Polish Botanical Journal 47: 91-101.

Pippola, E. \& Kotiranta, H. 2008. The genus Tremella (Basidiomycota, Tremellales) in Finland. Annales Botanici Fennici 45: 401-434.

Renvall, P. 1995. Community structure and dynamics of wood-rotting Basidiomycetes on decomposing conifer trunks in northern Finland. Karstenia 35: 1-51. https://doi.org/10.29203/ka.1995.309

Roberts, P. 1992. Spiral-spored Tulasnella species from Devon and the New Forest. Mycological Research 96: 233-236 https://doi. org/10.1016/S0953-7562(09)80973-8

Roberts, P. 1997. New Heterobasidiomycetes from Great Britain. Mycotaxon 63: 195-216.

Rödel, T. 2014. Über aktuelle Funde von Spiculogloea occulta P. Roberts, Spicugloea minuta P. Roberts und Spicugloea subminuta Hauerslev. Ein Beitrag zur Kenntnis der Gattung Spicugloea (Spiculogloeales, Agaricostilbomycetes). Zeitschrift für Mykologie 80: 491-504.

Rödel, T., Dämmrich, F. \& Kamke, M. 2020. Proterochaete adusta new to Germany. Boletus 41(1): 35-45

Runnel, K., Miettinen, O. \& Lõhmus, A. 2021. Polypore fungi as a flagship group to indicate changes in biodiversity - a test case from Estonia. IMA Fungus 12(1). https://doi.org/10.1186/s43008020-00050-y

Savola, K. 2015. The polypore survey of the forests in Helsinki-supplement 2014. Publications by City of Helsinki Environment Centre $1 / 2015$.

Savola, K. \& Kolehmainen, K. 2015 Pääkaupunkiseudun Viherkehän suojelualueiden käävät. Metsähallituksen luonnonsuojelujulkaisuja A 220: 1-65.
Siitonen, J., Hottola, J. \& Immonen, A. 2009. Differences in stand characteristics between brookside key habitats and managed forests in southern Finland. Silva Fennica 43: 21-37. https://doi. org/10.14214/sf.216

Spirin, V., Malysheva, V., Haelewaters, D., Larsson, K. H. 2019b. Studies in the Stypella vermiformis group. Antonie Van Leeuwenhoek 112: 753-764. https://doi.org/10.1007/s10482-018-01209-9

Spirin, V., Malysheva, V., Miettinen, O., Vlasak, J., Mendes Alvarenga, R. L., Gibertoni, T. B., Ryvarden, L \& Larsson, K-H 2019a. On Protomerulius and Heterochaetella (Auriculariales, Basidiomycota). Mycological Progress 18: 1079-1099. https://doi.org/10.1007/ s11557-019-01507-0

Spirin, V., Miettinen, O., Pennanen, J., Kotiranta, H. \& Niemelä, T. 2013. Antrodia hyalina, a new polypore from Russia, and A. leucaena, new to Europe. Mycological Progress 12: 53-61. https:// doi.org/10.1007/s11557-012-0815-0.

Spirin, V., Nordén, J., Svantesson, S. \& Larsson, K.-H. 2016. New records of intrahymenial heterobasidiomycetes (Basidiomycota) in north Europe. Nordic Journal of Botany 34: 475-477. https:// doi.org/10.1111/njb.01155

Stokland, J. \& Sippola, A. L. 2004. Monitoring protocol for wood-inhabiting fungi in the Alberta Biodiversity Monitoring Program. Alberta Biodiversity Monitoring Program, Alberta.

Svantesson, S., Larsson, K.-H., Kõljalg, U., May, T. W., Cangren, P., Nilsson, R. H. \& Larsson, E. 2019. Solving the taxonomic identity of Pseudotomentella tristis s.1. (Thelephorales, Basidiomycota) - a multi-gene phylogeny and taxonomic review, integrating ecological and geographical data. MycoKeys 50: 1-77. https://doi. org/10.3897/mycokeys.50.32432

Svrček, M. 1960. 'Tomentelloideae Čechoslovakiae. Genera resupinata familia Thelephoraceae'. Sydowia 14(1-6): 170-245.

Trichies, G. 2002. Serendipita lyrica sp. nov., Achroomyces lotharingus sp. nov. et quelques autres hétérobasidiés notables de Lorraine (France). Bulletin de la Société Mycologique de France 118(4): 351-379.

Vauras, J. 2000. Macrofungi of the Southwestern Archipelago National Park. Metsähallituksen luonnonsuojelujulkaisuja A 112: 1-91.

Venugopal, P., Julkunen-Tiitto, R., Junninen, K. \& Kouki, J. 2016. Phenolic compounds in Scots pine heartwood: are kelo trees a unique woody substrate? Canadian Journal of Forest Research 46: 225233. https://doi.org/10.1139/cjfr-2014-0498

Wells, K. \& Raitviir, A. 1966. Two new species of Exidiopsis. Eesti NSV Teaduste Akadeemia Toimetised. Bioloogiline seeria 15(2): 206-208. 